\title{
Pacific
}

Journal of

Mathematics

\section{STABLE TRACE FORMULAS AND DISCRETE SERIES} MULTIPLICITIES

STEVEn SPALLONE 


\title{
STABLE TRACE FORMULAS AND DISCRETE SERIES MULTIPLICITIES
}

\author{
STEVEN SPALlONE
}

Let $G$ be a reductive algebraic group over $\mathbb{Q}$, and suppose that $\Gamma \subset G(\mathbb{R})$ is an arithmetic subgroup defined by congruence conditions. A basic problem in arithmetic is to determine the multiplicities of discrete series representations in $L^{2}(\Gamma \backslash G(\mathbb{R}))$, and in general to determine the traces of Hecke operators on these spaces. In this paper we give a conjectural formula for the traces of Hecke operators, in terms of stable distributions. It is based on a stable version of Arthur's formula for $\boldsymbol{L}^{\mathbf{2}}$-Lefschetz numbers, which is due to Kottwitz. We reduce this formula to the computation of elliptic $p$-adic orbital integrals and the theory of endoscopic transfer. As evidence for this conjecture, we demonstrate the agreement of the central terms of this formula with the unipotent contributions to the multiplicity coming from Selberg's trace formula of Wakatsuki, in the case $G=\mathrm{GSp}_{4}$ and $\Gamma=\mathrm{GSp}_{4}(\mathbb{Z})$.

\section{Introduction}

Let $G$ be a reductive algebraic group over $\mathbb{Q}$, and $\Gamma$ an arithmetic subgroup of $G(\mathbb{R})$ defined by congruence conditions. Then $G(\mathbb{R})$ acts on $L^{2}(\Gamma \backslash G(\mathbb{R}))$ via right translation; let us write $R$ for this representation. A fundamental problem in arithmetic is to understand $R$. As a first step, we may decompose $R$ as

$$
R=R_{\text {disc }} \oplus R_{\text {cont }},
$$

where $R_{\text {disc }}$ is a direct sum of irreducible representations, and $R_{\text {cont }}$ decomposes continuously. The continuous part may be understood inductively through Levi subgroups of $G$ as in [Langlands 1976], leaving us with the study of $R_{\text {disc }}$. Given an irreducible representation $\pi$ of $G(\mathbb{R})$, write $R_{\text {disc }}(\pi)$ for the $\pi$-isotypic subspace of $R_{\text {disc. Then }}$

$$
R_{\mathrm{disc}}(\pi) \cong \pi^{\oplus m_{\mathrm{disc}}(\pi)}
$$

MSC2010: 11F46, 11F72, 22E55, 32N10.

Keywords: discrete series, Hecke operators, orbital integrals, Shimura varieties, endoscopy, fundamental lemma, stable trace formula. 
for some integer $m_{\mathrm{disc}}(\pi)$. (We may also write $m_{\mathrm{disc}}(\pi, \Gamma)$.) A basic problem is to compute these integers.

There is more structure than simply these dimensions, however. Arithmetic provides us with a multitude of Hecke operators $h$ on $L^{2}(\Gamma \backslash G(\mathbb{R}))$ that commute with $R$. Write $R_{\text {disc }}(\pi, h)$ for the restriction of $h$ to $R_{\text {disc }}(\pi)$. The general problem is to find a formula for the trace of $R_{\text {disc }}(\pi, h)$.

We focus on discrete series representations $\pi$. These are representations that behave like representations of compact or finite groups, in the sense that their associated matrix coefficients are square integrable. Like other smooth representations, they have a theory of characters developed by Harish-Chandra. They separate naturally into finite sets called $L$-packets. For an irreducible finite-dimensional algebraic representation $E$ of $G(\mathbb{C})$, there is a corresponding $L$-packet $\Pi_{E}$ of discrete series representations, consisting of those with the same infinitesimal and central characters as $E$.

We follow the tradition of computing $\operatorname{tr} R_{\text {disc }}(\pi, h)$ through trace formulas. This method has gone through several incarnations, beginning with Selberg [1956] for $\mathrm{GL}_{2}$, in which he also investigated the continuous Eisenstein series. A goal was to compute dimensions of spaces of modular forms, and traces of Hecke operators on these spaces. These spaces of modular forms correspond to the spaces $R_{\mathrm{disc}}(\pi)$ we are discussing in this case. His trace formula is an integral, over the quotient of the upper half space $X$ by $\Gamma$, of a sum of functions $H_{\gamma}$, one for each element of $\Gamma$. Let us write it roughly as

$$
\operatorname{dim}_{\mathbb{C}} S(\Gamma)=\int_{\Gamma \backslash X} \sum_{\gamma \in \Gamma} H_{\gamma}(Z) d Z,
$$

for some space $S(\Gamma)$ of cusp forms with a suitable $\Gamma$-invariance condition.

Here $d Z$ is a $G(\mathbb{R})$-invariant measure on $X$. When the quotient $\Gamma \backslash X$ is compact, the sum and integral may be interchanged, leading to a simple expression for the dimensions in terms of orbital integrals. The interference of the Eisenstein series precludes this approach in the noncompact quotient case. Here there are several convergence difficulties, which Selberg overcomes by employing a truncation process. Unfortunately the truncation process leads to notoriously complicated expressions, which are far from being in closed form. This study of $R_{\mathrm{disc}}(\pi)$ has been expanded to other reductive groups using what is called the Arthur-Selberg trace formula. See [Arthur 2005].

Generally, a trace formula is an equality of distributions on $G(\mathbb{R})$, or on the adelic group $G(\mathbb{A})$. One distribution is called the geometric side; it is a sum of terms corresponding to conjugacy classes of $G$. Given a test function $f$, the formula is essentially made up of combinations $I_{M}(\gamma, f)$ of weighted integrals of $f$ over the conjugacy classes of elements $\gamma$. (Here $M$ is a Levi subgroup of $G$.) 
The other distribution is called the spectral side, involving the Harish-Chandra transforms $\operatorname{tr} \pi(f)$ for various representations $\pi$. Here, the operator $\pi(f)$ is given by weighting the representation $\pi$ by $f$. The geometric and spectral sides agree, and in applications we can learn much about the latter from the former. Some of the art is in picking test functions to extract information about both sides.

The best general result using the trace formula to study $\operatorname{tr} R_{\text {disc }}(\pi, h)$ seems to be Arthur's [1989]. He produces a formula for

$$
\sum_{\pi \in \Pi} \operatorname{tr} R_{\text {disc }}(\pi, h)
$$

where $\Pi$ is a given discrete series $L$-packet for $G(\mathbb{R})$. He uses test functions $f$ which he calls "stable cuspidal". Their Fourier transforms $\pi \mapsto \operatorname{tr} \pi(f)$ are "stable" in that they are constant on $L$-packets, and "cuspidal" in that, considered as a function defined on tempered representations, they are supported on discrete series. (Tempered representations are those that appear in the Plancherel formula for $G(\mathbb{R})$.) Using his invariant trace formula, Arthur [1988a; 1988b] obtains (1-1) as the spectral side. The geometric side is a combination of orbital integrals for $h$ and values of Arthur's $\Phi$-function, which describes the asymptotic values of discrete series characters averaged over an $L$-packet.

In particular, he produces a formula for

$$
\sum_{\pi \in \Pi} m_{\mathrm{disc}}(\pi)
$$

for an $L$-packet $\Pi$ of (suitably regular) discrete series representations.

In the case of $G=\mathrm{GL}_{2}$, there is a discrete series representation $\pi_{k}$ for each integer $k \geq 1$. In this case $m_{\text {disc }}\left(\pi_{k}\right)$ is the dimension of the space $S_{k}(\Gamma)$ of $\Gamma$-cusp forms of weight $k$ on the upper half plane. Restriction to $\mathrm{SL}_{2}(\mathbb{R})$ gives two discrete series $\left\{\pi_{k}^{+}, \pi_{k}^{-}\right\}$in each $L$-packet. However we may still use Arthur's formula here since $m_{\text {disc }}\left(\pi_{k}^{+}, \Gamma\right)=m_{\text {disc }}\left(\pi_{k}^{-}, \Gamma\right)$ for every arithmetic subgroup $\Gamma$. (Endoscopy does not play a role.)

For the group $\mathrm{GSp}_{4}(\mathbb{R})$ there are two discrete series representations in each $L$ packet: one "holomorphic" and one "large" discrete series. Let $\pi$ be a holomorphic discrete series, and write $\pi^{\prime}$ for the large discrete series representation in the same $L$-packet as $\pi$. The multiplicity $m_{\text {disc }}(\pi, \Gamma)$ is also the dimension of a certain space of vector-valued Siegel cusp forms (see [Wallach 1984]) on the Siegel upper half space, an analogue of the usual cusp forms on the upper half plane. For $\Gamma=\mathrm{Sp}_{4}(\mathbb{Z})$, the dimensions of these spaces of cusp forms were calculated by Tsushima [1983; 1997] by using the Riemann-Roch-Hirzebruch formula, and later by Wakatsuki [2012] by using the Selberg trace formula and the properties of prehomogeneous 
vector spaces. In [ $\geq 2012]$, Wakatsuki then evaluated Arthur's formula to compute $m_{\text {disc }}(\pi, \Gamma)+m_{\text {disc }}\left(\pi^{\prime}, \Gamma\right)$, thereby deducing a formula for $m_{\text {disc }}\left(\pi^{\prime}, \Gamma\right)$.

A natural approach to isolating the individual $m_{\text {disc }}(\pi)$, or generally the individual tr $R_{\text {disc }}(\pi, h)$, is to apply a trace formula to a matrix coefficient, or more properly, a pseudocoefficient $f$. This means that $f$ is a test function whose Fourier transform picks out $\pi$ rather than the entire packet $\Pi$ containing $\pi$; see Definition 6 below. Such a function will not be stable cuspidal, but merely cuspidal. Arthur [1989] (see also [2005]) showed that $I_{M}(\gamma, f)$ vanishes when $f$ is stable cuspidal and the unipotent part of $\gamma$ is nontrivial. If we examine the geometric side of Arthur's formula for a pseudocoefficient $f$, we must evaluate the more complicated terms $I_{M}(\gamma, f)$ for elements $\gamma$ with nontrivial unipotent part. At the time of this writing, such calculations have not been made in general; we take another approach.

Distinguishing the individual representations $\pi$ from others in its $L$-packet leads to the theory of endoscopy, and stable trace formulas. The grouping of representations $\pi$ into packets $\Pi$ on the spectral side mirrors the fusion of conjugacy classes that occurs when one extends the group $G(\mathbb{R})$ to the larger group $G(\mathbb{C})$. If $F$ is a local or global field, then a stable conjugacy class in $G(F)$ is, roughly, the union of classes which become conjugate in $G(\bar{F})$. (See [Langlands 1979] for a precise definition.)

The distribution that takes a test function to its integral over a regular semisimple stable conjugacy class is a basic example of a stable distribution. Indeed, a stable distribution is defined to be a closure of the span of such distributions; see [Langlands $1983 ; 1979]$. A distribution on $G(F)$ is stabilized if it can be written as a sum of stable distributions, the sum being over smaller subgroups $H$ related to $G$. These groups $H$ are called endoscopic groups for $G$; they are tethered to $G$ not as subgroups but through their Langlands dual groups. As part of a series of techniques called endoscopy, one writes unstable distributions on $G$ as combinations of stable distributions on the groups $H$. Part of this process is the theory of transfer, associating suitable test functions $f^{H}$ on $H(F)$ to test functions $f$ on $G(F)$ that yield a matching of orbital integrals. Indeed this was the drive for [Ngô 2010]. As the name suggests, the theory of endoscopy, while laborious, leads to an intimate understanding of $G$.

There has been much work in stabilizing Arthur's formula. See for example [Langlands 1983; Arthur 2002; 2001; 2003]. In Kottwitz's preprint [ $\geq 2012$ ], he defines a stable version of Arthur's Lefschetz formula, which we review below. (See also [Morel 2010].) It is a combination $\mathscr{K}(f)=\sum_{H} \iota(G, H) S T_{g}\left(f^{H}\right)$ of distributions $f \mapsto f^{H} \mapsto S T_{g}\left(f^{H}\right)$ over endoscopic groups $H$ for $G$. Here the distributions $S T_{g}$, defined for each $H$, are stable. (See Section 5.1 for the definition of the rational numbers $\iota(G, H)$.) Each $S T_{g}$ is a sum of terms corresponding to 
stable conjugacy classes of elliptic elements $\gamma \in H(\mathbb{Q})$. Kottwitz's main result is that $\mathscr{K}$ agrees with Arthur's distribution, at least for functions $f$ that are stable cuspidal at the real place.

As part of the author's thesis [Spallone 2004], the identity terms of $\mathscr{K}$ were evaluated for the group $G=\mathrm{SO}_{5}$ at a function $f$ that was a pseudocoefficient for a discrete series representation at the real place. Later, Wakatsuki noted that the resulting expressions match up with the terms in his multiplicity formulas for $m_{\text {disc }}(\pi, \Gamma)$ and $m_{\text {disc }}\left(\pi^{\prime}, \Gamma\right)$ corresponding to unipotent elements. Moreover, the contribution in [Spallone 2004] from the endoscopic group accounted for the difference in these multiplicity formulas, while the stable part corresponded to the sum. After further investigation, we conjecture simply that Kottwitz's distribution evaluated at a function $f=f_{\pi, \Gamma}$ suitably adapted to $\pi$ and $\Gamma$ is equal to $m_{\text {disc }}(\pi, \Gamma)$, under a regularity condition on $\pi$. (See Section 5.3 for the precise statement.) Of course this is compatible with Arthur's results in [1989].

In this paper we give some computational evidence for this conjecture. We also reduce the computation of each $S T\left(f_{\pi, \Gamma}^{H}\right)$ to evaluating elliptic orbital $p$-adic integrals for the transfer $f^{\infty H}$ at the finite places. The rest breaks naturally into a problem at the real points and a global volume computation.

The main ingredient at the archimedean place is the $\Phi$-function $\Phi_{M}\left(\gamma, \Theta^{E}\right)$ of Arthur, which we review. This quantity gives the contribution from the real place to the trace formulas in [Arthur 1989] and [Goresky et al. 1997]. It also plays a prominent role in Kottwitz's formula. This function, originally defined by the asymptotic behavior of a stable character near a singular element $\gamma$, was expressed in closed form in many cases by the author in [Spallone 2009].

There are two volume-related constants that enter into any explicit computation of $S T_{g}$. The first is $\bar{v}(G)$, which is essentially the volume of an inner form of $G$ over $\mathbb{R}$. It depends on the choice of local measure $d g_{\infty}$. The second comes about from orbital integrals at the finite adeles, and depends on the choice of local measure $d g_{f}$. These integrals may frequently be written in terms of the volumes of open compact subgroups $K_{f}$ of $G\left(\mathbb{A}_{f}\right)$. In practice, one is left computing expressions such as $\bar{v}(G)^{-1} \operatorname{vol}_{d g_{f}}\left(K_{f}\right)^{-1}$, which are independent of the choice of local measures. More specifically, we define

$$
\chi_{K_{f}}(G)=\bar{v}(G)^{-1} \operatorname{vol}_{d g_{f}}\left(K_{f}\right)^{-1} \tau(G) d(G) .
$$

Here $\tau(G)$ is the Tamagawa number of $G$ and $d(G)$ is the index of the real Weyl group in the complex Weyl group. A main general result of this paper, Theorem 2, interprets $\chi_{K_{f}}(G)$ via Euler characteristics of arithmetic subgroups. It extends a computation of Harder [1971], which was for semisimple simply connected groups, to the case of reductive groups, under some mild hypotheses on $G$.

We work out two examples in this paper, one for $\mathrm{SL}_{2}$ and another for $\mathrm{GSp}_{4}$. It 
is easy to verify our conjecture for $G=\mathrm{SL}_{2}$ and $\Gamma=\mathrm{SL}_{2}(\mathbb{Z})$ using the classic dimension formula for cusp forms. In this case endoscopy does not appear. The calculations for $\mathrm{GSp}_{4}$ are more complex; we content ourselves with working out the central terms of Kottwitz's formula.

If $\pi$ is a holomorphic discrete series representation of $\mathrm{GSp}_{4}(\mathbb{R})$, write $H_{1}^{\pi}$ for the central-unipotent terms of the Selberg trace formula, as evaluated in [Wakatsuki $\geq 2012]$ to compute $m_{\text {disc }}(\pi, \Gamma)$. Here $\Gamma=\mathrm{GSp}_{4}(\mathbb{Z})$. If $\pi$ is a large discrete series representation, write $H_{1}^{\pi}$ for the central-unipotent terms in [Wakatsuki $\geq 2012$ ] contributing to $m_{\text {disc }}(\pi, \Gamma)$. In both cases, write $f=f_{\pi, \Gamma}=f_{\infty} f^{\infty}$, with $f_{\infty}$ a pseudocoefficient for $\pi$, and $f^{\infty}$ the (normalized) characteristic function of the integer adelic points of $G$. Write $\mathscr{K}(f, \pm 1)$ for the central terms of Kottwitz's formula applied to $f$.

As evidence for our conjecture, we show this:

Theorem 1. For each regular discrete series representation $\pi$ of $G(\mathbb{R})$, we have

$$
\mathscr{K}\left(f_{\pi, \Gamma}, \pm 1\right)=H_{1}^{\pi} .
$$

We believe that the $\mathscr{K}\left(f_{\pi, \Gamma}, \pm 1\right)$ terms will generally match up with the difficult central-unipotent terms of the Arthur-Selberg formula, as in this case.

Our conjecture reduces the computation of discrete series multiplicities to the computation of stable elliptic orbital integrals of various transfers $f_{p}^{H}$, written for functions on $G\left(\mathbb{Q}_{p}\right)$. Let us write this as $S O_{\gamma_{H}}\left(f_{p}^{H}\right)$. Here $f_{p}$ are characteristic functions of congruence subgroups of $G\left(\mathbb{Q}_{p}\right)$ related to $\Gamma$. Certainly at suitably regular elements, $S O_{\gamma_{H}}\left(f_{p}^{H}\right)$ is an unstable combination of orbital integrals of $f_{p}$; however there are also contributions from elliptic singular $\gamma_{H}$, notably $\gamma_{H}=1$. At present, there are expressions for $f_{p}^{H}$ in the parahoric case and of course for $G\left(\mathbb{Z}_{p}\right)$, but less seems to be known for smaller congruence subgroups. On the other hand, there are many formulas for dimensions of Siegel cusp forms and discrete series multiplicities for these cases (for example, [Wakatsuki $\geq 2012]$ ). This suggests that one could predict stable singular elliptic orbital integrals $S O_{\gamma_{H}}\left(f_{p}^{H}\right)$ for the transfer $f_{p}^{H}$ of characteristic functions of congruence subgroups (see for example Klingen, Iwahori and Siegel), by comparing our formulas.

Finally, we refer the casual reader to our survey [Spallone 2011] of the present approach to discrete series multiplicities.

In Section 2, we set up the conventions for this study. We explain how we are setting up the orbital integrals, and indicate our main computational tools. We also review the Langlands correspondence for real groups.

The theory of Arthur's $\Phi$-function is reviewed in Section 4. In Section 5, we review Kottwitz's stable version of Arthur's formula from [Kottwitz $\geq 2012$ ]. We also state our conjecture here. The heart of the volume computations in this paper 
is in Section 6, where we determine $\chi_{K}(G)$. As a warm up, we work out the classic case of $\mathrm{SL}_{2}$, with $\Gamma=\mathrm{SL}_{2}(\mathbb{Z})$ in Section 7 .

The case of $G=\mathrm{GSp}_{4}$ is considerably more difficult. We must work out several isomorphisms of real tori. These are described in Section 8. The basic structure of $G$ and its Langlands dual $\hat{G}$ is set up in Section 9. In Section 10 we work out the Langlands parameters for discrete series of $G(\mathbb{R})$. There is only one elliptic endoscopic group $H$ for $G$. We describe $H$ in Section 11. In Section 12, we describe the Langlands parameters for discrete series of $H(\mathbb{R})$ and describe the transfer of discrete series in this case. In Section 13, we describe the Levi subgroups of $G$ and $H$ and compute various constants that occur in Kottwitz's formula for these groups. In Section 14, we compute explicitly Arthur's $\Phi$-function for Levi subgroups of $G$, and we do this for Levi subgroups of $H$ in Section 15. In Section 16, we write out the terms of Kottwitz's formula corresponding to central elements of $G$ and $H$, for a general arithmetic subgroup $\Gamma$. In Section 17, we specialize to the case of $\Gamma=\mathrm{GSp}_{4}(\mathbb{Z})$, and in Section 18 we gather our results to demonstrate Theorem 1.

\section{Preliminaries and notation}

If $F$ is a field, write $\Gamma_{F}$ for the absolute Galois group of $F$. Suppose $G$ is an algebraic group over $F$. If $E$ is an extension field of $F$, we write $G_{E}$ for $G$ viewed as an algebraic group over $E$ (by restriction). If $\gamma$ is an element of $G(F)$, we denote by $G_{\gamma}$ the centralizer of $\gamma$ in $G$. By $G^{\circ}$ we denote the identity component of $G$ (with the Zariski topology). Write $G_{\text {der }}$ for the derived group of $G$. If $G$ is a reductive group, write $G_{\text {sc }}$ for the simply connected cover of $G_{\text {der }}$. Let $X^{*}(G)=\operatorname{Hom}\left(G_{\bar{F}}, \mathbb{G}_{m}\right)$ and $X_{*}(G)=\operatorname{Hom}\left(\mathbb{G}_{m}, G_{\bar{F}}\right)$. These are abelian groups. Write $X^{*}(G)_{\mathbb{C}}$ and $X_{*}(G)_{\mathbb{C}}$ for the tensor product of these groups over $\mathbb{Z}$ with $\mathbb{C}$. Similarly with the subscript $\mathbb{R}$. Write $A_{G}$ for the maximal $F$-split torus in the center of $G$.

We denote by $\mathbb{A}$ the ring of adeles over $\mathbb{Q}$. We denote by $\mathbb{A}_{f}$ the ring of finite adeles over $\mathbb{Q}$, so that $\mathbb{A}=\mathbb{A}_{f} \times \mathbb{R}$. Write $\mathscr{O}_{f}$ for the integral points of $\mathbb{A}_{f}$.

If $G$ is a real Lie group, we write $G^{+}$for the connected component of $G$ (using the classical topology rather than any Zariski topology).

Let $G$ be a connected reductive group over $\mathbb{R}$. A torus $T$ in $G$ is elliptic if $T / A_{G}$ is anisotropic (as an $\mathbb{R}$-torus). Say that $G$ is cuspidal if it contains a maximal torus $T$ that is elliptic. An element of $G(\mathbb{R})$ is elliptic if it is contained in an elliptic maximal torus of $G$. Having fixed an elliptic maximal torus $T$, the absolute Weyl group $\Omega_{G}$ of $T$ in $G$ is the quotient of the normalizer of $T(\mathbb{C})$ in $G(\mathbb{C})$ by $T(\mathbb{C})$. The real Weyl group $\Omega_{G, \mathbb{R}}$ of $T$ in $G$ is the quotient of the normalizer of $T(\mathbb{R})$ in $G(\mathbb{R})$ by $T(\mathbb{R})$. We may drop the subscript $G$ if it is clear from context. Also fix a maximal compact subgroup $K_{\mathbb{R}}$ of $G(\mathbb{R})$. 
Write $q(G)$ for half the dimension of $G(\mathbb{R}) / K_{\mathbb{R}} Z(\mathbb{R})$. If we write $R$ for the roots of $G$, with a set of positive roots $R^{+}$, then

$$
q(G)=\frac{1}{2}\left(\left|R^{+}\right|+\operatorname{dim}(X)\right),
$$

where $X$ is the span of $R$.

If $G$ is an algebraic group over $\mathbb{Q}$, let $G(\mathbb{Q})^{+}=G(\mathbb{R})^{+} \cap G(\mathbb{Q})$.

2.1. Endoscopy. Here we review the theory of based root data and endoscopy in the form we will use in this paper.

The notion of a based root datum is defined in [Springer 1979]. First, a root datum is a quadruple $\Psi=\left(X, R, X^{\vee}, R^{\vee}\right)$, where

- $X$ and $X^{\vee}$ are free, finitely generated abelian groups, in duality by a pairing

$$
\langle\cdot, \cdot\rangle: X \times X^{\vee} \rightarrow \mathbb{Z}
$$

- $R$ and $R^{\vee}$ are finite subsets of $X$ and $X^{\vee}$, respectively;

- there is a bijection $\alpha \mapsto \alpha^{\vee}$ from $R$ onto $R^{\vee}$;

- we have $\left\langle\alpha, \alpha^{\vee}\right\rangle=2$ for all $\alpha \in R$;

- $s_{\alpha}(R)=R$ if $s_{\alpha}$ is the reflection of $X$ determined by $\alpha$, and similarly with $\alpha$ replaced by $\alpha^{\vee}$ and $R$ by $R^{\vee}$.

A based root datum is a quadruple $\Psi_{0}=\left(X, \Delta, X^{\vee}, \Delta^{\vee}\right)$, where $\Delta$ and $\Delta^{\vee}$ are sets of simple roots of root system $R$ and $R^{\vee}$ respectively, so that $\left(X, R, X^{\vee}, R^{\vee}\right)$ is a root datum. The dual of $\Psi_{0}=\left(X, \Delta, X^{\vee}, \Delta^{\vee}\right)$ is given simply by $\Psi_{0}^{\vee}=$ $\left(X^{\vee}, \Delta^{\vee}, X, \Delta\right)$.

Let $\Psi_{0}=\left(X, \Delta, X^{\vee}, \Delta^{\vee}\right)$ and $\Psi_{0}^{\prime}=\left(X^{\prime}, \Delta^{\prime}, X^{\prime \vee}, \Delta^{\prime \vee}\right)$ be two based root data. Then an isomorphism between $\Psi$ and $\Psi^{\prime}$ is an isomorphism of groups $f: X \rightarrow X^{\prime}$ so that $f$ induces a bijection of $\Delta$ onto $\Delta^{\prime}$ and so that the transpose of $f$ induces a bijection of $\Delta^{\vee}$ onto $\Delta^{\prime v}$.

Let $G$ be a connected reductive group over an algebraically closed field $F$. Fix a maximal torus $T$ and a Borel subgroup $B$ of $G$ with $T \subseteq B$. We say in this situation that $(T, B)$ is a pair (for $G)$. The choice of pair determines a based root datum

$$
\Psi_{0}(G, T, B)=\left(X^{*}(T), \Delta(T, B), X_{*}(T), \Delta^{\vee}(T, B)\right)
$$

for $G$. Here $\Delta(T, B)$ is the set of simple $B$-positive roots of $T$, and $\Delta^{\vee}(T, B)$ is the set of simple $B$-positive coroots of $T$. If another pair $T^{\prime} \subseteq B^{\prime}$ is chosen, the new based root datum obtained is canonically isomorphic to the original via an inner automorphism $\alpha$ of $G$. We have $\alpha\left(T^{\prime}\right)=T$ and $\alpha\left(B^{\prime}\right)=B$. Although the inner automorphism $\alpha$ need not be unique, its restriction to an isomorphism $T^{\prime} \stackrel{\sim}{\rightarrow} T$ is unique. 
We may remove the dependence of the based root datum on the choice of pair as follows. Write $X^{*}, \Delta, X_{*}$, and $\Delta^{\vee}$ for the inverse limit over the set of pairs $(T, B)$ of $X^{*}(T), \Delta(T, B), X_{*}(T)$ and $\Delta^{\vee}(T, B)$, respectively. Then we simply define the based root datum of $G$ to be

$$
\Psi_{0}(G)=\left(X^{*}, \Delta, X_{*}, \Delta^{\vee}\right) .
$$

Let $G$ be a connected reductive group over a field $F$, and $\Psi_{0}(G)$ a based root datum of $G_{\bar{F}}$. Then $\Gamma_{F}$ acts naturally (via isomorphisms) on $\Psi_{0}(G)$. The action of $\Gamma_{F}$ on $G$ is said to be an $L$-action if it fixes some splitting of $G$; see [Kottwitz 1984, Section 1.3].

Definition 1. A dual group for $G$ is the following data:

(i) A connected complex reductive group with a based root datum $\Psi_{0}(\hat{G})$. We write its complex points as $\hat{G}$.

(ii) An $L$-action of $\Gamma_{F}$ on $\hat{G}$.

(iii) $\mathrm{A} \Gamma_{F}$-isomorphism from $\Psi_{0}(\hat{G})$ to the dual of $\Psi_{0}(G)$.

To specify the isomorphism for (iii) above, one typically fixes pairs $\left(T_{0}, B_{0}\right)$ of $G$ and $\left(\hat{S}_{0}, \hat{B}_{0}\right)$ of a dual group $\hat{G}$ and an isomorphism from $\Psi_{0}\left(\hat{G}, \hat{S}_{0}, \hat{B}_{0}\right)$ to the dual of $\Psi_{0}\left(G, T_{0}, B_{0}\right)$.

In the case that $G$ is a torus $T$, the dual group $\hat{T}$ is simply given by

$$
\hat{T}=X^{*}(T) \otimes_{\mathbb{Z}} \mathbb{C}^{\times},
$$

with the $\Gamma_{F}$-action induced from $X^{*}(T)$. There are canonical $\Gamma_{F}$-isomorphisms $X^{*}(\hat{T}) \stackrel{\sim}{\rightarrow} X_{*}(T)$ and $X_{*}(\hat{T}) \stackrel{\sim}{\rightarrow} X^{*}(T)$.

The formalism for dual groups encodes canonical isomorphisms between tori. If $T$ and $T^{\prime}$ are tori, and $\varphi: T \rightarrow T^{\prime}$ is a homomorphism, it induces a homomorphism $\hat{T}^{\prime} \rightarrow \hat{T}$ in the evident way.

Suppose that $(T, B)$ is a pair for $G$ and $(\hat{S}, \hat{B})$ is a pair for $\hat{G}$. By (iii) above, one has in particular a fixed isomorphism from $\Psi_{0}(G, T, B)$ to the dual of $\Psi_{0}(\hat{G}, \hat{S}, \hat{B})$. In particular this yields an isomorphism from $X^{*}(T)$ to $X_{*}(\hat{S})$, which induces an isomorphism

$$
\hat{T} \stackrel{\sim}{\rightarrow} \hat{S}
$$

Next, let $G$ be a connected reductive group over a field $F$, which is either local or global.

Definition 2. An endoscopic group for $G$ is a triple $(H, s, \eta)$ as follows:

- $H$ is a quasisplit connected group, with a fixed dual group $\hat{H}$ as above;

- $s \in Z(\hat{H})$. 
- $\eta: \hat{H} \rightarrow \hat{G}$ is an embedding.

- The image of $\eta$ is $(\hat{G})_{\eta(s)}^{\circ}$, the connected component of the centralizer in $\hat{G}$ of $\eta(s)$.

- The $\hat{G}$-conjugacy class of $\eta$ is fixed by $\Gamma_{F}$.

Cohomology of $\Gamma_{F}$-modules then yields a boundary map

$$
[Z(\hat{H}) / Z(\hat{G})]^{\Gamma_{F}} \rightarrow H^{1}(F, Z(\hat{G})) .
$$

- The image of $s$ in $Z(\hat{H}) / Z(\hat{G})$ is fixed by $\Gamma$, and its image under the boundary map above is trivial if $F$ is local and locally trivial if $F$ is global.

An endoscopic group is elliptic if the identity components of $Z(\hat{G})^{\Gamma_{F}}$ and $Z(\hat{H})^{\Gamma_{F}}$ agree.

Isomorphism of endoscopic groups is defined in [Kottwitz 1984, Section 7.5]; we do not review it here.

2.2. Langlands correspondence. Let $G$ be a connected reductive group over $\mathbb{R}$. In this section we review elliptic Langlands parameters for $G$ and the corresponding $L$-packets for discrete series representations of $G(\mathbb{R})$. Our main references are [Borel 1979] and [Kottwitz 1990]. Write $W_{\mathbb{R}}$ for the Weil group of $\mathbb{R}$, and $W_{\mathbb{C}}$ for the canonical image of $\mathbb{C}^{\times}$in $W_{\mathbb{R}}$. There is an exact sequence

$$
1 \rightarrow W_{\mathbb{C}} \rightarrow W_{\mathbb{R}} \rightarrow \Gamma_{\mathbb{R}} \rightarrow 1 .
$$

The Weil group $W_{\mathbb{R}}$ is generated by $W_{\mathbb{C}}$ and a fixed element $\tau$ satisfying $\tau^{2}=-1$ and $\tau z \tau^{-1}=\bar{z}$ for $z \in W_{\mathbb{C}}$. The action of $\Gamma_{\mathbb{R}}$ on $\hat{G}$ inflates to an action of $W_{\mathbb{R}}$ on $\hat{G}$, and through this action we form the $L$-group ${ }^{L} G=\hat{G} \rtimes W_{\mathbb{R}}$.

A Langlands parameter $\varphi$ for $G$ is an equivalence class of continuous homomorphisms $\varphi: W_{\mathbb{R}} \rightarrow{ }^{L} G$ commuting with projection to $\Gamma_{\mathbb{R}}$, satisfying a mild hypothesis on the image; see [Borel 1979]. The equivalence relation is via inner automorphisms from $\hat{G}$. One associates to a Langlands parameter $\varphi$ an $L$-packet $\Pi(\varphi)$ of irreducible admissible representations of $G$.

Suppose that $G$ is cuspidal, so that there is a discrete series representation of $G(\mathbb{R})$. This implies that the longest element $w_{0}$ of the Weyl group $\Omega$ acts as -1 on $X_{*}(T)$. If $\varphi$ is a Langlands parameter, write $C_{\varphi}$ for the centralizer of $\varphi\left(W_{\mathbb{R}}\right)$ in $\hat{G}$ and $\hat{S}$ for the centralizer of $\varphi\left(W_{\mathbb{C}}\right)$ in $\hat{G}$. Write $S_{\varphi}$ for the product $C_{\varphi} Z(\hat{G})$. We say $\varphi$ is elliptic if $S_{\varphi} / Z(\hat{G})$ is finite, and describe the $L$-packet $\Pi(\varphi)$ in this case.

Since $\varphi$ is elliptic, the centralizer $\hat{S}$ is a maximal torus in $\hat{G}$. Since $\varphi$ commutes with the projection to $\Gamma_{\mathbb{R}}$, it restricts to a homomorphism

$$
W_{\mathbb{C}} \rightarrow \hat{S} \times\{1\} .
$$


We may view this restriction as a continuous homomorphism $\varphi: \mathbb{C}^{\times} \rightarrow \hat{S}$, which may be written in exponential form

$$
\varphi(z)=z^{\mu} \bar{z}^{v}
$$

with $\mu$ and $v$ regular elements of $X_{*}(\hat{T})_{\mathbb{C}}$. Write $\hat{B}$ for the unique Borel subgroup of $\hat{G}$ containing $\hat{S}$ so that $\langle\mu, \alpha\rangle$ is positive for every root $\alpha$ of $\hat{S}$ that is positive for $\hat{B}$. We say that $\varphi$ determines the pair $(\hat{S}, \hat{B})$, at least up to conjugacy in $\hat{G}$.

Let $B$ be a Borel subgroup of $G_{\mathbb{C}}$ containing $T$. Then $\varphi$ and $B$ determine a quasicharacter $\chi_{B}=\chi(\varphi, B)$, as follows. There is a canonical (up to $\hat{G}$-conjugacy) homomorphism $\eta_{B}:{ }^{L} T \rightarrow{ }^{L} G$ described in [Kottwitz 1990] such that

$$
\eta_{B}(z)=z^{\rho} \bar{z}^{-\rho} \times z \in \hat{G} \rtimes W_{\mathbb{R}} \text { for } z \in W_{\mathbb{C}} .
$$

Here $\rho=\rho_{G}$ is the half sum of the $B$-positive roots for $T$. Then a Langlands parameter $\varphi_{B}$ for $T$ may be chosen so that $\varphi=\eta_{B} \circ \varphi_{B}$. Finally $\chi_{B}$ is the quasicharacter associated to $\varphi_{B}$ by the Langlands correspondence for $T$ (as described in [Borel 1979, Section 9.4]).

Write $\mathscr{B}$ for the set of Borels of $G_{\mathbb{C}}$ containing $T$. The $L$-packet associated to $\varphi$ is indexed by $\Omega_{\mathbb{R}} \backslash \mathscr{B}$. For $B \in \Omega_{\mathbb{R}} \backslash \mathscr{B}$, a representation $\pi(\varphi, B)$ in the $L$-packet is given by the irreducible discrete series representation of $G(\mathbb{R})$ whose character $\Theta_{\pi}$ is given on regular elements $\gamma$ of $T(\mathbb{R})$ by

$$
(-1)^{q(G)} \sum_{\omega \in \Omega_{\mathbb{R}}} \chi_{\omega(B)}(\gamma) \cdot \Delta_{\omega(B)}(\gamma)^{-1} .
$$

Here $\Delta_{B}$ is the usual discriminant

$$
\Delta_{B}(\gamma)=\prod_{\alpha>0 \text { for } B}\left(1-\alpha(\gamma)^{-1}\right) .
$$

Finally, let

$$
\Pi(\varphi)=\left\{\pi(\varphi, B) \mid B \in \Omega_{\mathbb{R}} \backslash \mathscr{B}\right\} .
$$

It has order $d(G)=\left|\Omega / \Omega_{\mathbb{R}}\right|$. There is a unique irreducible finite-dimensional algebraic complex representation $E$ of $G(\mathbb{C})$ with the same infinitesimal character and central character as the representations in this $L$-packet. It has highest weight $\mu-\rho \in X^{*}(T)$ with respect to $B$. The isomorphism classes of such $E$ are in oneto-one correspondence with elliptic Langlands parameters $\varphi$, and we often write $\Pi_{E}$ for $\Pi(\varphi)$.

Definition 3. We say that a discrete series representation $\pi \in \Pi_{E}$ is regular if the highest weight of $E$ is regular. 
2.3. Measures and orbital integrals. Let $G$ be a locally compact group with Haar measure $d g$. If $f$ is a continuous function on $G$, write $f d g$ for the measure on $G$ given by

$$
\varphi \mapsto \int_{G} \varphi(g) f(g) d g,
$$

for $\varphi$ continuous and compactly supported in $G$. We will refer to the measures obtained in this way simply as "measures". If $G$ is a $p$-adic, real, or adelic Lie group, we require that $f$ be suitably smooth.

In this paper, we will view orbital integrals and Fourier transforms as distributions defined on measures, rather than on functions. This approach eases their dependence on choices of local measures, choices that do not matter in the end.

For $K$ an open compact subset of $G$, write $e_{K}$ for the measure given by $f d g$, where $f$ is the characteristic function of $K$ divided by $\operatorname{vol}_{d g}(K)$. Note that the measure $e_{K}$ is independent of the choice of Haar measure $d g$.

Let $G$ be a reductive group defined over a local field $F$. Fix a Haar measure $d g$ on $G(F)$. Let $f d g$ be a measure on $G(F)$, and take a semisimple element $\gamma \in G(F)$. Fix a Haar measure $d t$ of $G(F)_{\gamma}^{\circ}$. Then we write $O_{\gamma}(f d g ; d t)$ for the usual orbital integral

$$
O_{\gamma}(f d g ; d t)=\int_{G_{\gamma^{\circ}(F) \backslash G(F)}} f\left(g^{-1} \gamma g\right) \frac{d g}{d t} .
$$

Many cases of finite orbital integrals are easy to compute by the following result, a special case extracted from [Kottwitz 1986, Section 7].

Proposition 1. Let $F$ be a p-adic field with ring of integers $\mathcal{O}$. Let $G$ be a split connected reductive group defined over $\mathbb{O}$, and let $K=G(\mathcal{O})$. Suppose that $\gamma \in K$ is semisimple, and that $1-\alpha(\gamma)$ is either 0 or a unit for every root $\alpha$ of G. Let $\gamma^{\prime}$ be stably conjugate to $\gamma$. Then $O_{\gamma^{\prime}}\left(e_{K} ; d t\right)$ vanishes unless $\gamma^{\prime}$ is conjugate to $\gamma$, in which case

$$
O_{\gamma^{\prime}}\left(e_{K} ; d t\right)=\operatorname{vol}_{d t}\left(G_{\gamma}{ }^{\circ}(F) \cap K\right)^{-1} .
$$

Now let $G$ be a reductive group defined over $\mathbb{Q}$.

Let $f^{\infty} d g_{f}$ be a measure on $G\left(\mathbb{A}_{f}\right)$ and take a semisimple element $\gamma \in G\left(\mathbb{A}_{f}\right)$. Fix a Haar measure $d t_{f}$ of $G_{\gamma}^{\circ}\left(\mathbb{A}_{f}\right)$. Write $O_{\gamma}\left(f^{\infty} d g_{f} ; d t_{f}\right)$ for the orbital integral

$$
O_{\gamma}\left(f^{\infty} d g_{f} ; d t_{f}\right)=\int_{G_{\gamma}{ }^{\circ}\left(\mathbb{A}_{f}\right) \backslash G\left(\mathbb{A}_{f}\right)} f^{\infty}\left(g^{-1} \gamma g\right) \frac{d g_{f}}{d t_{f}} .
$$

We also have the stable orbital integrals

$$
S O_{\gamma}\left(f^{\infty} d g_{f} ; d t_{f}\right)=\sum_{i} e\left(\gamma_{i}\right) O_{\gamma_{i}}\left(f^{\infty} d g_{f} ; d t_{i, f}\right),
$$


the sum being over $\gamma_{i} \in G\left(\mathbb{A}_{f}\right)$ (up to $G\left(\mathbb{A}_{f}\right)$-conjugacy) whose local components are stably conjugate to $\gamma$. The centralizers of $\gamma$ and a given $\gamma_{i}$ are inner forms of each other, and we use corresponding measures $d t_{f}$ and $d t_{i, f}$. The number $e\left(\gamma_{i}\right)$ is defined as follows: For a reductive group $A$ over a local field, Kottwitz [1983] has defined an invariant $e(A)$. It is equal to 1 if $A$ is quasisplit. For each place $v$ of $\mathbb{Q}$, write $\gamma_{i, v}$ for the $v$ th component of $\gamma_{i}$. Let

$$
e\left(\gamma_{i, v}\right)=e\left(G_{\gamma_{i, v}}^{\circ}\left(\mathbb{Q}_{v}\right)\right) \text {. }
$$

Finally, let

$$
e\left(\gamma_{i}\right)=\prod_{v} e\left(\gamma_{i, v}\right)
$$

Definition 4. Let $M$ be a Levi component of a parabolic subgroup $P$ of $G$, and $d m_{f}$ a Haar measure on $M\left(\mathbb{A}_{f}\right)$. Given a measure $f^{\infty} d g_{f}$, its $M$-constant term is the measure $f_{M}^{\infty} d m_{f}$, where $f_{M}^{\infty}$ is defined via

$$
f_{M}^{\infty}(m)=\delta_{P\left(\mathbb{A}_{f}\right)}^{-1 / 2}(m) \int_{N\left(\mathbb{A}_{f}\right)} \int_{K_{f}} f^{\infty}\left(k^{-1} n m k\right) d k_{f} d n_{f}
$$

Here we fix the Haar measure $d k_{f}$ on $K_{f}$ giving it mass one, and the Haar measure $d n_{f}$ on $N\left(\mathbb{A}_{f}\right)$ is chosen so that $d g_{f}=d k_{f} d n_{f} d m_{f}$. The function $\delta_{P\left(\mathbb{A}_{f}\right)}$ is the modulus function on $P\left(\mathbb{A}_{f}\right)$.

It is independent of the choice of parabolic subgroup $P$.

Proposition 2. Let $G$ be a split group defined over $\mathbb{Z}$ and let $K_{f}=G\left(\mathcal{O}_{f}\right)$. Then

$$
\left(e_{K_{f}}\right)_{M}=e_{M\left(\mathbb{A}_{f}\right) \cap K_{f}} .
$$

Proof. Write $e_{K_{f}}=f^{\infty} d g_{f}$. Then it is easy to see that $f_{M}^{\infty}(m)=0$ unless $m \in K_{f}$. If $m \in K_{f}$, we compute that

$$
f_{M}^{\infty}(m)=\frac{\operatorname{vol}_{d k_{f}}\left(K_{f}\right) \operatorname{vol}_{d n_{f}}\left(K_{f} \cap N\left(\mathbb{A}_{f}\right)\right)}{\operatorname{vol}_{d g_{f}}\left(K_{f}\right)} .
$$

The result follows since

$$
\operatorname{vol}_{d g_{f}}\left(K_{f}\right)=\operatorname{vol}_{d m_{f}}\left(M\left(\mathbb{A}_{f}\right) \cap K_{f}\right) \operatorname{vol}_{d n_{f}}\left(N\left(\mathbb{A}_{f}\right) \cap K_{f}\right) \operatorname{vol}_{d k_{f}}\left(K_{f}\right) .
$$

2.4. Pseudocoefficients. We continue with a connected reductive group $G$ over $\mathbb{Q}$, and adopt some terminology from [Arthur 1989]. Fix a maximal compact subgroup $K_{\mathbb{R}}$ of $G(\mathbb{R})$. We put $K_{\mathbb{R}}^{\prime}=K_{\mathbb{R}} A_{G}(\mathbb{R})^{+}$. Given a quasicharacter (smooth homomorphism to $\left.\mathbb{C}^{\times}\right) \xi$ on $A_{G}(\mathbb{R})^{+}$, write $\mathscr{H}_{\mathrm{ac}}(G(\mathbb{R}), \xi)$ for the space of smooth, $K_{\mathbb{R}}^{\prime}$-finite functions on $G(\mathbb{R})$ that are compactly supported modulo $A_{G}(\mathbb{R})^{+}$, and 
transform under $A_{G}(\mathbb{R})^{+}$according to $\xi$. Write $\Pi(G(\mathbb{R}), \xi)$ for the set of irreducible representations of $G(\mathbb{R})$ whose central character restricted to $A_{G}(\mathbb{R})^{+}$is equal to $\xi$.

Given a function $f \in \mathscr{H}_{\mathrm{ac}}\left(G(\mathbb{R}), \xi^{-1}\right)$, a representation $\pi \in \Pi(G(\mathbb{R}), \xi)$, and a Haar measure $d g_{\infty}$ on $G(\mathbb{R})$, write $\pi\left(f d g_{\infty}\right)$ for the operator on the space of $\pi$ given by the formula

$$
\pi\left(f d g_{\infty}\right)=\int_{G(\mathbb{R}) / A_{G}(\mathbb{R})^{+}} f(x) \pi(x) d g_{\infty} .
$$

Here we give $A_{G}(\mathbb{R})^{+}$the measure corresponding to Lebesgue measure on $\mathbb{R}^{n}$, if $A_{G}$ is $n$-dimensional. The operator is of trace class.

Write $\Pi_{\text {temp }}(G(\mathbb{R}), \xi)$ (respectively $\Pi_{\text {disc }}(G(\mathbb{R}), \xi)$ ) for the subset of tempered (respectively discrete series) representations in $\Pi(G(\mathbb{R}), \xi)$.

Definition 5. Suppose that $f \in \mathscr{H}_{\mathrm{ac}}\left(G(\mathbb{R}), \xi^{-1}\right)$. We say that the measure $f d g_{\infty}$ is cuspidal if $\operatorname{tr} \pi\left(f d g_{\infty}\right)$, viewed as a function on $\Pi_{\text {temp }}(G(\mathbb{R}), \xi)$, is supported on $\Pi_{\text {disc }}(G(\mathbb{R}), \xi)$.

Write $\tilde{E}$ for the contragredient of the representation $E$. Arthur [1989] employs functions $f_{E} \in \mathscr{H}_{\mathrm{ac}}\left(G(\mathbb{R}), \xi^{-1}\right)$ with $f_{E} d g_{\infty}$ cuspidal, whose defining property is that, for all $\pi \in \Pi_{\text {temp }}(G(\mathbb{R}), \xi)$,

$$
\operatorname{tr} \pi\left(f_{E} d g_{\infty}\right)= \begin{cases}(-1)^{q(G)} & \text { if } \pi \in \Pi_{\tilde{E}}, \\ 0 & \text { otherwise. }\end{cases}
$$

Such measures can be broken down further.

Definition 6. Fix a representation $\pi_{0} \in \Pi_{\text {disc }}\left(G(\mathbb{R}), \xi^{-1}\right)$, and suppose that $f_{0} \in$ $\mathcal{H}_{\mathrm{ac}}\left(G(\mathbb{R}), \xi^{-1}\right)$. Suppose the measure $f_{0} d g_{\infty}$ satisfies, for all $\pi \in \Pi_{\mathrm{temp}}(G(\mathbb{R}), \xi)$,

$$
\operatorname{tr} \pi\left(f_{0} d g_{\infty}\right)= \begin{cases}(-1)^{q(G)} & \text { if } \pi \cong \tilde{\pi}_{0} \\ 0 & \text { otherwise }\end{cases}
$$

It follows from the corollary in [Clozel and Delorme 1984, Section 5.2] that such functions exist. Pick such a function $f_{0}$, and put $e_{\pi_{0}}=f_{0} d g_{\infty}$.

Suppose that for each $\pi \in \Pi_{E}$ we fix measures $e_{\pi}$ as above. Let

$$
f_{E} d g_{\infty}=\sum_{\pi} e_{\pi}
$$

the sum being over $\pi \in \Pi_{E}$. Then clearly $f_{E} d g_{\infty}$ satisfies Arthur's condition (2-3).

We remark that the measure $(-1)^{q(G)} e_{\pi}$ is called a pseudocoefficient of $\tilde{\pi}$. 


\section{Transfer}

We sketch the important theory of transfer in the form that we will use in this paper.

Suppose that $G$ is a real connected reductive group, and that $(H, s, \eta)$ is an elliptic endoscopic group for $G$. Fix an elliptic maximal torus $T_{H}$ of $H$, an elliptic maximal torus $T$ of $G$, and an isomorphism $j: T_{H} \stackrel{\sim}{\rightarrow} T$ between them. Also fix a Borel subgroup $B$ of $G_{\mathbb{C}}$ containing $T$ and a Borel subgroup $B_{H}$ of $H_{\mathbb{C}}$ containing $T_{H}$.

Suppose that $\xi$ is a quasicharacter on $A_{G}(\mathbb{R})$, and that $f_{\infty} \in \mathscr{H}_{\mathrm{ac}}\left(G(\mathbb{R}), \xi^{-1}\right)$, with $f_{\infty} d g_{\infty}$ cuspidal. There is a corresponding quasicharacter $\xi_{H}$ on $A_{H}(\mathbb{R})$ described in [Kottwitz $\geq 2012$, Section 5.5].

There is also a measure $f_{\infty}^{H} d h_{\infty}$ on $H(\mathbb{R})$ with $f_{\infty}^{H} \in \mathscr{H}_{\mathrm{ac}}\left(H(\mathbb{R}), \xi_{H}^{-1}\right)$, having matching character values. See [Shelstad 1982; Clozel and Delorme 1984; 1990; Langlands and Shelstad 1987]. More specifically, let $\varphi_{H}$ be a tempered Langlands parameter for $H_{\mathbb{R}}$, and write $\Pi_{H}=\Pi\left(\varphi_{H}\right)$ for the corresponding $L$-packet of discrete series representations of $H(\mathbb{R})$. Transport $\varphi_{H}$ via $\eta$ to a tempered Langlands parameter $\varphi_{G}$ for $G$. The parameters $\varphi_{G}$ and $\varphi_{H}$ determine pairs $(\hat{S}, \hat{B})$ and $\left(\hat{S}_{H}, \hat{B}_{H}\right)$ as in Section 2.2.

Then

$$
\operatorname{tr} \Pi_{H}\left(f_{\infty}^{H} d h_{\infty}\right)=\sum_{\pi \in \Pi} \Delta_{\infty}\left(\varphi_{H}, \pi\right) \cdot \operatorname{tr} \pi\left(f_{\infty} d g_{\infty}\right),
$$

using Shelstad's transfer factors $\Delta_{\infty}\left(\varphi_{H}, \pi\right)$. Both sides of (3-1) vanish unless $\Pi_{H}$ is a discrete series packet. In particular, $f_{\infty}^{H} d h_{\infty}$ is cuspidal, and it may be characterized by (3-1). (The transfer $f_{\infty}^{H} d h_{\infty}$ is only defined up to the kernel of stable distributions.) We may use this formula to identify it as a combination of pseudocoefficients.

It is a delicate matter to specify the transfer factors. We will use a formula for $\Delta_{\infty}\left(\varphi_{H}, \pi\right)$ from [Kottwitz 1990], which is itself a reformulates a formula from [Shelstad 1982]. One must carefully specify the duality between $G$ and $\hat{G}$, and between $H$ and $\hat{H}$, because this factor depends on precisely how this is done. It also depends on the isomorphism $j: T_{H} \widetilde{\rightarrow} T$, which must be compatible with correspondences of tori determined by the Langlands parameters, as specified below.

Definition 7. The triple $\left(j, B_{T}, B_{T_{H}}\right)$ is aligned with $\varphi_{H}$ if the following diagram commutes:

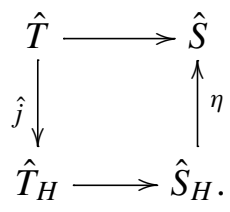


Here the isomorphisms $\hat{T} \rightarrow \hat{S}$ and $\hat{T}_{H} \rightarrow \hat{S}_{H}$ are determined, as in (2-2), by $(B, \hat{B})$ and $\left(B_{H}, \hat{B}_{H}\right)$, respectively. The map $\hat{j}$ is the map dual to $j$ using the identification (2-1) of the dual tori.

For each $\omega \in \Omega$, there is a character

$$
a_{\omega}:(\hat{T} / Z(\hat{G}))^{\Gamma_{\mathbb{R}}} \rightarrow\{ \pm 1\}
$$

described in [Kottwitz 1990].

If the triple $\left(j, B_{T}, B_{T_{H}}\right)$ is aligned with $\varphi_{H}$, then we may take as transfer factors

$$
\Delta_{\infty}\left(\varphi_{H}, \pi\left(\varphi, \omega^{-1}(B)\right)\right)=\left\langle a_{\omega}, \hat{j}^{-1}(s)\right\rangle .
$$

Next, let $G$ be a connected reductive algebraic group over $\mathbb{Q}$, and let $(H, s, \eta)$ be an endoscopic group for $G$. Given a measure $f^{\infty} d g_{f}$ on $G\left(\mathbb{A}_{f}\right)$, there is a measure $f^{\infty H} d h_{f}$ on $H\left(\mathbb{A}_{f}\right)$ such that for all $\gamma_{H} \in H\left(\mathbb{A}_{f}\right)$ suitably regular, one has

$$
S O_{\gamma_{H}}\left(f^{\infty H} d h_{f}\right)=\sum_{\gamma} \Delta^{\infty}\left(\gamma_{H}, \gamma\right) O_{\gamma}\left(f^{\infty} d g_{f}\right)
$$

The sum is taken over $G\left(\mathbb{A}_{f}\right)$-conjugacy classes of "images" $\gamma \in G\left(\mathbb{A}_{f}\right)$ of $\gamma_{H}$. We have written $\Delta^{\infty}\left(\gamma_{H}, \gamma\right)$ for the Langlands-Shelstad transfer factors. One takes matching measures on the centralizers of $\gamma_{H}$ and the various $\gamma$ in forming the quotient measures for the orbital integrals. We have left out many details; please see [Langlands and Shelstad 1987] and [Kottwitz and Shelstad 1999] for definitions, and [Ngô 2010] for the celebrated proof.

\section{Arthur's $\Phi$-function}

In this section we consider a reductive group $G$ defined over $\mathbb{R}$. Let $T$ be a maximal torus contained in a Borel subgroup $B$ of $G_{\mathbb{C}}$. Let $A$ be the split part of $T$, let $T_{c}$ be the maximal compact subtorus of $T$, and let $M$ be the centralizer of $A$ in $G$. It is a Levi subgroup of $G$ containing $T$. Let $E$ be an irreducible finite-dimensional (algebraic) representation of $G(\mathbb{C})$, and consider the $L$-packet $\Pi_{E}$ of discrete series representations $\pi$ of $G(\mathbb{R})$ that have the same infinitesimal and central characters as $E$. Write $\Theta_{\pi}$ for the character of $\pi$, and put

$$
\Theta^{E}=(-1)^{q(G)} \sum_{\pi \in \Pi_{E}} \Theta_{\pi} .
$$

Note that $\Theta^{E}(\gamma)$ will not extend continuously to all elements $\gamma \in T(\mathbb{R})$, and in particular not to $\gamma=1$. Define the function $D_{M}^{G}$ on $T$ by

$$
D_{M}^{G}(\gamma)=\operatorname{det}(1-\operatorname{Ad}(\gamma) ; \operatorname{Lie}(G) / \operatorname{Lie}(M)) .
$$


Then a result of Arthur and Shelstad [Arthur 1989] states that the function

$$
\gamma \mapsto\left|D_{M}^{G}(\gamma)\right|^{1 / 2} \Theta^{E}(\gamma)
$$

defined on the set of regular elements $T_{\text {reg }}(\mathbb{R})$, extends continuously to $T(\mathbb{R})$. We denote this extension by $\Phi_{M}\left(\gamma, \Theta^{E}\right)$. The following closed expression for $\Phi_{M}\left(\gamma, \Theta^{E}\right)$ when $\gamma \in T_{c}$ is given in [Spallone 2009].

Proposition 3. If $\gamma \in T_{c}(\mathbb{R})$, then

$$
\Phi_{M}\left(\gamma, \Theta^{E}\right)=(-1)^{q(L)}\left|\Omega_{L}\right| \sum_{\omega \in \Omega^{L M}} \varepsilon(\omega) \operatorname{tr}\left(\gamma ; V_{\omega\left(\lambda_{B}+\rho_{B}\right)-\rho_{B}}^{M} .\right.
$$

In particular,

(i) if $T$ is compact, then $M=G$ and $\Phi_{G}\left(\gamma, \Theta^{E}\right)=\operatorname{tr}(\gamma ; E)$;

(ii) if $T$ is split, then $M=A$ and $\Phi_{A}\left(1, \Theta^{E}\right)=(-1)^{q(G)}\left|\Omega_{G}\right|$.

The notation needs to be explained. Here $L$ is the centralizer of $T_{c}$ in $G$. The roots of $T$ in $L$ and $M$ are the real and imaginary roots, respectively, of $T$ in $G$. Write $\Omega_{L}$ and $\Omega_{M}$ for the respective Weyl groups. Write $\Omega^{L M}$ for the set of elements that are simultaneously Kostant representatives for both $L$ and $M$, relative to $B$. We write $\varepsilon$ for the sign character of $\Omega_{G}$. Finally by $V_{\omega\left(\lambda_{B}+\rho_{B}\right)-\rho_{B}}^{M}$ we denote the irreducible finite-dimensional representation of $M(\mathbb{C})$ with highest weight $\omega\left(\lambda_{B}+\rho_{B}\right)-\rho_{B}$, where $\lambda_{B}$ is the $B$-dominant highest weight of $E$.

If $z \in G(\mathbb{R})$ is central, it is easy to see that $\Phi_{M}\left(\gamma z, \Theta^{E}\right)=\lambda_{E}(z) \Phi_{M}\left(\gamma, \Theta^{E}\right)$, where $\lambda_{E}$ is the central character of $E$. Thus, for the case of central $\gamma=z$, computing $\Phi_{M}\left(z, \Theta^{E}\right)$ amounts to computing the dimensions of finite-dimensional representations of $M(\mathbb{C})$ with various highest weights. For this we use the Weyl dimension formula, in the following form.

Proposition 4 (Weyl dimension formula). Let $G$ be a complex reductive group and $T$ a maximal torus in $G$, contained in a Borel subgroup $B$. Write $\rho_{B}$ for the half-sum of the positive roots for $T$ in $G$ (with respect to $B$ ). Let $\lambda_{B} \in X^{*}(T)$ be a positive weight. Then there is a unique irreducible representation $V_{\lambda_{B}}$ of $G$ with highest weight $\lambda_{B}$. Its dimension is given by

$$
\operatorname{dim}_{\mathbb{C}} V_{\lambda_{B}}=\prod_{\alpha>0} \frac{\left\langle\alpha, \lambda_{B}+\rho_{B}\right\rangle}{\left\langle\alpha, \rho_{B}\right\rangle} .
$$

Here $\langle\cdot, \cdot\rangle$ is a nondegenerate $\Omega_{G}$-invariant inner product on $X^{*}(T)_{\mathbb{R}}$, which is unique up to a scalar. 


\section{Kottwitz's formula}

5.1. Various invariants. In this section we introduce some invariants involved in Kottwitz's formula.

By $\bar{G}$ we generally denote an inner form of $G_{\mathbb{R}}$ such that $\bar{G} / A_{G}$ is anisotropic over $\mathbb{R}$.

Definition 8. Let $G$ be a cuspidal reductive group over $\mathbb{R}$, and $d g_{\infty}$ a Haar measure on $G(\mathbb{R})$. Let

$$
\bar{v}\left(G ; d g_{\infty}\right)=e(\bar{G}) \operatorname{vol}\left(\bar{G}(\mathbb{R}) / A_{G}(\mathbb{R})^{+}\right) .
$$

This is a stable version of the constant $v(G)$ that appears in [Arthur 1989]. As before, $e(\bar{G})$ is the sign defined in [Kottwitz 1983]. (Note that $e(\bar{G})=(-1)^{q(G)}$ when $G$ is quasisplit.) In both cases the Haar measure on $\bar{G}(\mathbb{R})$ is transported from $d g_{\infty}$ on $G(\mathbb{R})$ in the usual way, and the measure on $A_{G}(\mathbb{R})^{+}$is the standard Lebesgue measure.

Definition 9. Let $G$ be a cuspidal connected reductive group over $\mathbb{Q}$. Then $G$ contains a maximal torus $T$ such that $T / A_{G}$ is anisotropic over $\mathbb{R}$. Write $T_{\text {sc }}$ for the inverse image in $G_{\mathrm{sc}}$ of $T$. Then $k(G)$ is the cardinality of the image of $H^{1}\left(\mathbb{R}, T_{\mathrm{sc}}\right) \rightarrow H^{1}(\mathbb{R}, T)$.

Definition 10. If $G$ is a reductive group over $\mathbb{Q}$, write $\tau(G)$ for the Tamagawa number of $G$, as defined in [Ono 1966].

By [Kottwitz 1988] or [Kottwitz $\geq 2012$ ], the Tamagawa numbers $\tau(G)$ for a reductive group $G$ over $\mathbb{Q}$ may be computed using the formula

$$
\tau(G)=\left|\pi_{0}\left(Z(\hat{G})^{\Gamma_{\mathbb{Q}}}\right)\right| \cdot\left|\operatorname{ker}^{1}(\mathbb{Q}, Z(\hat{G}))\right|^{-1} .
$$

Here $\pi_{0}$ denotes the topological connected component.

Definition 11. Let $M$ be a Levi subgroup of $G$. Then put

$$
n_{M}^{G}=\left[N_{G}(M)(\mathbb{Q}): M(\mathbb{Q})\right] .
$$

Here $N_{G}(M)$ denotes the normalizer of $M$ in $G$.

Definition 12. Let $\gamma \in M(\mathbb{Q})$ be semisimple. Then put

$$
\bar{\iota}^{M}(\gamma)=\left|\left(M_{\gamma} / M_{\gamma}^{\circ}\right)(\mathbb{Q})\right| \quad \text { and } \quad \iota^{M}(\gamma)=\left[M_{\gamma}(\mathbb{Q}): M_{\gamma}^{\circ}(\mathbb{Q})\right] .
$$

Let $(H, s, \eta)$ be an endoscopic triple for $G$, and write $\operatorname{Out}(H, s, \eta)$ for its outer automorphisms. Put

$$
\iota(G, H)=\tau(G) \tau(H)^{-1}|\operatorname{Out}(H, s, \eta)|^{-1} .
$$


5.2. The formula. In this section we give Kottwitz's formula $[\geq 2012]$.

Our $G$ will now be a cuspidal connected reductive group over $\mathbb{Q}$. Let $f^{\infty} \in$ $C_{c}^{\infty}\left(G\left(\mathbb{A}_{f}\right)\right)$ and $f_{\infty} \in \mathscr{H}_{\mathrm{ac}}(G(\mathbb{R}), \xi)$ for some $\xi$. We consider measures $f d g$ of the form $f d g=f^{\infty} d g_{f} \cdot f_{\infty} d g_{\infty} \in C_{c}^{\infty}(G(\mathbb{A}))$, for some decomposition $d g=d g_{f} d g_{\infty}$ of the Tamagawa measure on $G\left(\mathbb{A}_{f}\right)$. Also choose such decompositions for every cuspidal Levi subgroup $M$ of $G$.

First we define the stable distribution $S \Phi_{M}$ at the archimedean place:

Definition 13. Let $M$ be a cuspidal Levi subgroup of $G$. Let $\gamma \in M(\mathbb{Q})$ be elliptic, and pick a Haar measure $d t_{\infty}$ of $M_{\gamma}^{\circ}(\mathbb{R})$. Then $S \Phi_{M}\left(\gamma, f_{\infty} d g_{\infty} ; d t_{\infty}\right)$ is defined to be

$$
(-1)^{\operatorname{dim}\left(A_{M} / A_{G}\right)} k(M) k(G)^{-1} \bar{v}\left(M_{\gamma}^{\circ} ; d t_{\infty}\right)^{-1} \sum_{\Pi} \Phi_{M}\left(\gamma^{-1}, \Theta_{\Pi}\right) \operatorname{tr} \Pi\left(f_{\infty} d g_{\infty}\right),
$$

the sum being taken over $L$-packets of discrete series representations.

Here is the basic building block of Kottwitz's formula:

Definition 14. Let $M$ be a cuspidal Levi subgroup of $G$, and $\gamma \in M(\mathbb{Q})$ an elliptic element. Pick Haar measures $d t_{f}$ on $M_{\gamma}^{\circ}\left(\mathbb{A}_{f}\right)$ and $d t_{\infty}$ on $M_{\gamma}^{\circ}(\mathbb{R})$ whose product is the Tamagawa measure $d t$ on $M_{\gamma}^{\circ}(\mathbb{A})$.

We define

$S T_{g}(f d g, \gamma, M)$

$$
=\left(n_{M}^{G}\right)^{-1} \tau(M) \bar{\iota}^{M}(\gamma)^{-1} S O_{\gamma}\left(f_{M}^{\infty} d m_{f} ; d t_{f}\right) S \Phi_{M}\left(\gamma, f_{\infty} d g_{\infty} ; d t_{\infty}\right) .
$$

Here $f_{M}^{\infty} d m_{f}$ is the $M$-constant term of $f^{\infty} d g_{f}$. The product

$$
S O_{\gamma}\left(f_{M}^{\infty} d m_{f} ; d t_{f}\right) \bar{v}\left(M ; d t_{\infty}\right)
$$

is independent of the decompositions of $d t$ and $d g$. We will therefore often write this simply as $S O_{\gamma}\left(f_{M}^{\infty} d m_{f}\right) \bar{v}(M)$, and similarly for other such products.

Kottwitz defines

$$
S T_{g}(f d g)=\sum_{M} \sum_{\gamma \in M} S T_{g}(f d g, \gamma, M) .
$$

Here $M$ runs over $G(\mathbb{Q})$-conjugacy classes of cuspidal Levi subgroups in $G$, and the second sum runs over stable $M(\mathbb{Q})$-conjugacy classes of semisimple elements $\gamma \in M(\mathbb{Q})$ that are elliptic in $M(\mathbb{R})$.

For convenience we also define, for $\gamma \in G(\mathbb{Q})$ semisimple,

$$
S T_{g}(f d g, \gamma)=\sum_{M} S T_{g}(f d g, \gamma, M),
$$

the sum being taken over cuspidal Levi subgroups of $G$ with semisimple $\gamma \in M(\mathbb{Q})$ that are elliptic in $M(\mathbb{R})$. 
Kottwitz's stable version of Arthur's trace formula is given by

$$
\mathscr{K}(f d g)=\sum_{(H, s, \eta) \in \mathscr{E}_{0}} \iota(G, H) S T_{g}\left(f^{H} d h\right),
$$

where $\mathscr{E}_{0}$ is the set of (equivalence classes of) elliptic endoscopic groups for $G$.

We record here the simpler form of $S T_{g}(f d g, \gamma, M)$ when $\gamma=z$ is in the rational points $Z(\mathbb{Q})$ of the center of $G$. We have

$S T_{g}(f d g, z, M)$

$$
=(-1)^{\operatorname{dim}\left(A_{M} / A_{G}\right)} \frac{k(M)}{k(G)}\left(n_{M}^{G}\right)^{-1} \tau(M) f_{M}^{\infty}(z) \bar{v}\left(M ; d m_{\infty}\right)^{-1} \Phi_{M}\left(z^{-1}, \Theta_{\Pi}\right) .
$$

5.3. Conjecture. Recall the stable cuspidal measure $f_{E} d g_{\infty}$ from Section 2.4. Fix any test function $f^{\infty} d g_{f}$ and put $f=f^{\infty} f_{E} d g$.

Let

$$
T_{g}(f d g)=\sum_{M}\left(n_{M}^{G}\right)^{-1} \sum_{\gamma} \iota^{M}(\gamma)^{-1} \tau\left(M_{\gamma}\right) O_{\gamma}\left(f_{M}^{\infty} d m_{f}\right) \Phi_{M}\left(\gamma, f_{E} d g_{\infty}\right) .
$$

Again, the sum is over cuspidal Levi subgroups $M$ and semisimple $\gamma \in M(\mathbb{Q})$ that are elliptic in $M(\mathbb{R})$. Here as in [Arthur 1989], $\Phi_{M}(\gamma, \cdot)$ is the unnormalized form of the distribution $I_{M}$ defined in [Arthur 1988a].

Now suppose that $\pi \in \Pi_{\text {disc }}(G(\mathbb{R}), \xi)$, and let $K_{f}$ be an open compact subgroup of $G\left(\mathbb{A}_{f}\right)$. Write

$$
L^{2}\left(G(\mathbb{Q}) \backslash G(\mathbb{A}) / K_{f}, \xi\right)
$$

for the space of functions on this double coset space that transform by $A_{G}(\mathbb{R})^{+}$ according to $\xi$ and are square integrable modulo center. Write $R_{\text {disc }}\left(\pi, K_{f}\right)$ for the $\pi$-isotypical subspace of $L^{2}\left(G(\mathbb{Q}) \backslash G(\mathbb{A}) / K_{f}, \xi\right)$; it is finite-dimensional. If $f^{\infty} d g_{f}$ is $K_{f}$-biinvariant, then convolution gives an operator $R_{\text {disc }}\left(\pi, f^{\infty} d g_{f}\right)$ on $R_{\text {disc }}\left(\pi, K_{f}\right)$. According to [Arthur 1989, Corollary 6.2], if the highest weight of $E$ is regular, then

$$
\sum_{\pi \in \Pi_{E}} \operatorname{tr} R_{\mathrm{disc}}\left(\pi, f^{\infty} d g_{f}\right)=T_{g}(f d g) .
$$

The main result of [Kottwitz $\geq 2012$ ] is that if $f_{\infty} d g_{\infty}$ is stable cuspidal, then $T_{g}(f d g)=\mathscr{K}(f d g)$. Since we may assume $f_{E} d g_{\infty}=\sum_{\pi \in \Pi_{E}} e_{\pi}$, the following conjecture is plausible:

Conjecture 1. Fix a regular discrete series representation $\pi$ of $G(\mathbb{R})$. As in Section 2.4, let $f_{\infty} d g_{\infty}=e_{\pi}$. Pick a measure $f^{\infty} d g_{f}$ with $f^{\infty} \in C_{c}\left(G\left(\mathbb{A}_{f}\right)\right)$, and $d g_{f} d g_{\infty}=d g$ the Tamagawa measure on $G(\mathbb{A})$. Put $f=f^{\infty} f_{\infty}$. Then

$$
\mathscr{K}(f d g)=\operatorname{tr} R_{\mathrm{disc}}\left(\pi, f^{\infty} d g_{f}\right) .
$$


In particular, if we choose a compact open subgroup $K_{f}$ of $G\left(\mathbb{A}_{f}\right)$, and put $f^{\infty} d g_{f}=e_{K_{f}}$, we obtain

$$
m_{\mathrm{disc}}\left(\pi, K_{f}\right)=\mathscr{K}\left(e_{\pi} e_{K_{f}}\right) .
$$

In this paper we give some evidence for this conjecture. Moreover, we will see that $\mathscr{K}(f d g)$ is given by a closed algebraic expression, which is straightforward to evaluate, so long as one can compute the transfers $e_{\pi}^{H}$ at the real place, and evaluate the semisimple orbital integrals of $f^{\infty H} d h_{f}$ at the finite adeles.

\section{Euler characteristics}

We have finished our discussion of Kottwitz's formula, and now solve the arithmetic volume problem mentioned in the introduction. For simplicity we will write $K$ rather than $K_{f}$ for open compact subgroups of $G\left(\mathbb{A}_{f}\right)$ in this section.

Definition 15. For $K$ a compact open subgroup of $G\left(\mathbb{A}_{f}\right)$, we define

$$
\chi_{K}(G)=\bar{v}\left(G ; d g_{\infty}\right)^{-1} \operatorname{vol}_{d g_{f}}(K)^{-1} \tau(G) d(G)
$$

if $G$ is cuspidal. If $G$ is not cuspidal, then $\chi_{K}(G)=0$.

Note that if $K_{0}$ is another compact open subgroup of $G\left(\mathbb{A}_{f}\right)$, with $K \subseteq K_{0}$ of finite index, then $\chi_{K}(G)=\left[K_{0}: K\right] \chi_{K_{0}}(G)$. In this section we compute the quantities $\chi_{K}(G)$ under some mild hypotheses on $G$.

6.1. Statement of theorem. Before getting embroiled in details, let us sketch the idea of the computation of $\chi_{K}(G)$. The computation is considerably easier if $K$ is sufficiently small. In this case, $\chi_{K}(G)$ is the classical Euler characteristic of a Shimura variety. This in turn may be written in terms of Euler characteristics of an arithmetic subgroup of $G_{\mathrm{ad}}(\mathbb{R})$. For $G$ a semisimple and simply connected Chevalley group, such Euler characteristics were computed in [Harder 1971].

Our work is to reduce to this case. Given a compact open subgroup $K_{0}$ of $G\left(\mathbb{A}_{f}\right)$, we will pick a sufficiently small subgroup $K$ of $K_{0}$. By the above we know the analogue of $\chi_{K}(G)$ for $G^{\text {sc }}$. To compute $\chi_{K_{0}}(G)$ we have two tasks: to change between $G$ and $G^{\text {sc }}$, and to change between $K$ and $K_{0}$.

The resulting formula entails several standard definitions:

Definition 16. Write $G(\mathbb{R})_{+} \subseteq G(\mathbb{R})$ for the inverse image of $G_{\text {ad }}(\mathbb{R})^{+}$. Let $G(\mathbb{Q})_{+}=G(\mathbb{Q}) \cap G(\mathbb{R})_{+}$. Write $v: G \rightarrow C$ for the quotient of $G$ by $G_{\text {der. }}$. Let $C(\mathbb{R})^{\dagger}=v(Z(\mathbb{R}))$, and $C(\mathbb{Q})^{\dagger}=C(\mathbb{Q}) \cap C(\mathbb{R})^{\dagger}$. Write $\rho: G_{\mathrm{sc}} \rightarrow G_{\mathrm{der}}$ for the usual covering of $G_{\mathrm{der}}$ by $G_{\mathrm{sc}}$. For $K$ a compact open subgroup of $G\left(\mathbb{A}_{f}\right)$, let $K^{\text {der }}=G_{\text {der }}\left(\mathbb{A}_{f}\right) \cap K$, and let $K^{\text {sc }}$ be the preimage of $K$ in $G_{\mathrm{sc}}\left(\mathbb{A}_{f}\right)$. Let $\Gamma_{K}=G(\mathbb{Q})_{+} \cap K$, let $\Gamma_{K}^{\text {der }}=G_{\text {der }}(\mathbb{Q})_{+} \cap K$, let $\Gamma_{K}^{\mathrm{sc}}=K^{\mathrm{sc}} \cap G_{\mathrm{sc}}(\mathbb{Q})_{+}$, and write $\Gamma_{K}^{\mathrm{ad}}$ for the image of $\Gamma_{K}$ in $G_{\mathrm{ad}}(\mathbb{Q})$. 
In this section we avoid certain awkward tori for simplicity, preferring the following kind:

Definition 17. A torus $T$ over $\mathbb{Q}$ is $\mathbb{Q R}$-equitropic if the largest $\mathbb{Q}$-anisotropic torus in $T$ is $\mathbb{R}$-anisotropic.

Here are some basic facts about $\mathbb{Q} \mathbb{R}$-equitropic tori.

Proposition 5. If $T$ is a $\mathbb{Q} \mathbb{R}$-equitropic torus, then $T(\mathbb{Q})$ is discrete in $T\left(\mathbb{A}_{f}\right)$. If $G$ is a reductive group, and the connected component $Z^{\circ}$ of the center of $G$ is $\mathbb{Q R}$-equitropic, then its derived quotient $C$ is also $\mathbb{Q R}$-equitropic.

Proof. The first statement follows from [Milne 2005, Theorem 5.26]. The second is straightforward.

Serre [1971] introduces an Euler characteristic $\chi_{\mathrm{alg}}(\Gamma) \in \mathbb{Q}$ applicable to any group $\Gamma$ with a finite index subgroup $\Gamma_{0}$ that is torsion-free and has finite cohomological dimension. In particular, it applies to our congruence subgroups $\Gamma=\Gamma_{K}$. Here are some simple properties of $\chi_{\text {alg }}$ :

- For an exact sequence of the form

$$
1 \rightarrow A \rightarrow B \rightarrow C \rightarrow 1
$$

with $A, B$ and $C$ groups as above, we have $\chi_{\text {alg }}(B)=\chi_{\text {alg }}(A) \cdot \chi_{\text {alg }}(C)$.

- If $\Gamma$ is a finite group, then $\chi_{\mathrm{alg}}(\Gamma)=|\Gamma|^{-1}$.

The theorem of this section relates $\chi_{K}(G)$ to $\chi_{\mathrm{alg}}\left(\Gamma_{K}^{\mathrm{sc}}\right)$. More precisely:

Theorem 2. Let $G$ be a reductive group over $\mathbb{Q}$. Assume that $G_{\mathrm{sc}}$ has no compact factors and that the connected component $Z^{\circ}$ of the center of $G$ is $\mathbb{Q R}$-equitropic. Let $K_{0} \subset G\left(\mathbb{A}_{f}\right)$ be a compact open subgroup. Then $\chi_{K_{0}}(G)$ is equal to

$$
\begin{aligned}
& |\operatorname{ker}(\rho(\mathbb{Q}))|\left[G_{\mathrm{der}}\left(\mathbb{A}_{f}\right): G_{\mathrm{der}}(\mathbb{Q})_{+} K_{0}^{\mathrm{der}}\right] \\
& \frac{\cdot\left[\Gamma_{K_{0}}^{\text {der }}: G_{\mathrm{der}}(\mathbb{Q})_{+} \cap \rho\left(K_{0}^{\mathrm{sc}}\right)\right]\left[C\left(\mathbb{A}_{f}\right): C(\mathbb{Q})^{\dagger} v\left(K_{0}\right)\right]}{\left[G(\mathbb{R}): G(\mathbb{R})_{+}\right]\left|v\left(K_{0}\right) \cap C(\mathbb{Q})^{\dagger}\right|} \chi_{\mathrm{alg}}\left(\Gamma_{K_{0}}^{\mathrm{sc}}\right) .
\end{aligned}
$$

Here $\rho(\mathbb{Q})$ denotes the map $\rho(\mathbb{Q}): G_{\mathrm{sc}}(\mathbb{Q}) \rightarrow G(\mathbb{Q})$ on $\mathbb{Q}$-points. The assumption on the absence of compact factors is needed for strong approximation, and is discussed in [Milne 2005].

When $G_{\text {sc }}$ is a Chevalley group and $\Gamma_{K_{0}}^{\mathrm{sc}}=G_{\mathrm{sc}}(\mathbb{Z})$, this reduces the problem to the calculation of Harder [1971]:

Proposition 6. Let $G$ be a simply connected, semisimple Chevalley group over $\mathbb{Z}$. Write $m_{1}, \ldots, m_{r}$ for the exponents of its Weyl group $\Omega$, and put $\Gamma=G(\mathbb{Z})$. We have

$$
\chi_{\mathrm{alg}}(\Gamma)=\left(-\frac{1}{2}\right)^{r}\left|\Omega_{\mathbb{R}}\right|^{-1} \prod_{i=1}^{r} B_{m_{i}+1} .
$$


Here $B_{n}$ denotes the $n$-th Bernoulli number. Recall that $\Omega_{\mathbb{R}}$ is the real Weyl group of $G$.

6.2. Shimura varieties. To prove Theorem 2, we will use some basic Shimura variety theory, which may be found in [Deligne 1979] or [Milne 2005]. Much of the theory holds only for $K$ sufficiently small. For simplicity, we will say " $K$ is small" rather than " $K$ is a sufficiently small finite index subgroup of $K_{0}$ ".

For convenience, we gather here many simplifying properties of small $K$, which we will often use without comment. For the rest of this section assume that $Z(G)^{\circ}$ is $\mathbb{Q} \mathbb{R}$-equitropic, and that $G_{\mathrm{sc}}$ has no compact factors.

Proposition 7. Let $K$ be small.

(i) $K \cap Z(\mathbb{Q})=\{1\}$.

(ii) $v(K) \cap C(\mathbb{Q})=\{1\}$.

(iii) $G(\mathbb{Q}) \cap K G_{\mathrm{der}}\left(\mathbb{A}_{f}\right) \subseteq G_{\mathrm{der}}(\mathbb{Q})$.

(iv) $G_{\mathrm{der}}\left(\mathbb{A}_{f}\right) \cap G(\mathbb{Q}) K=G_{\mathrm{der}}(\mathbb{Q}) K_{\mathrm{der}}$.

(v) $K \cap G_{\mathrm{der}}(\mathbb{Q}) \subseteq \rho\left(G_{\mathrm{sc}}(\mathbb{Q})\right)$.

(vi) $K \cap G(\mathbb{Q}) \subseteq G(\mathbb{Q})^{+}$.

Proof. The first two items follow because $Z^{\circ}$ and thus $C$ are $\mathbb{Q R R}$-equitropic. Item (iii) follows from [Deligne 1979, Corollaire 2.0.12], and the next is a corollary. Items (v) and (vi) follow from [Deligne 1979, Corollaire 2.0.5 and 2.0.14], respectively.

Recall that we have chosen a maximal compact subgroup $K_{\mathbb{R}}$ of $G(\mathbb{R})$.

Definition 18. Let

$$
X=G(\mathbb{R}) / K_{\mathbb{R}}^{+} Z(\mathbb{R}), \quad \bar{X}=G(\mathbb{R}) / K_{\mathbb{R}} Z(\mathbb{R}), \quad S_{K}=G(\mathbb{Q}) \backslash X \times G\left(\mathbb{A}_{f}\right) / K
$$

be the double coset space obtained through the action $q(x, g) k=(q x, q g k)$ of $q \in G(\mathbb{Q})$ and $k \in K$.

Similarly, let

$$
\bar{S}_{K}=G(\mathbb{Q}) \backslash \bar{X} \times G\left(\mathbb{A}_{f}\right) / K,
$$

with the action of $G(\mathbb{Q}) \times K$ defined in the same way.

The component group of $S_{K}$ is finite and given (see [Deligne 1979, 2.1.3]) by

$$
\pi_{0}\left(S_{K}\right)=G\left(\mathbb{A}_{f}\right) / G(\mathbb{Q})_{+} K .
$$

There is some variation in the literature regarding the use of $X$ versus $\bar{X}$. Deligne [1979] and Milne [2005] implicitly use $X$ (in light of Deligne's [Proposition 1.2.7]). Harder [1971] uses $\bar{X}$. Arthur [1989] uses

$$
G(\mathbb{R}) / K_{\mathbb{R}}^{\prime} .
$$


(Recall that $K_{\mathbb{R}}^{\prime}=A_{G}(\mathbb{R})^{+} K_{\mathbb{R}}$.) Since for us $Z^{\circ}$ is $\mathbb{Q} \mathbb{R}$-equitropic, we have

$$
K_{\mathbb{R}}^{\prime}=Z(\mathbb{R}) K_{\mathbb{R}},
$$

and so this quotient is equal to $\bar{X}$.

Since we would like to combine results stated in terms of $X$ with others stated in terms of $\bar{X}$, we must understand the precise relationship between the two. This is the purpose of Proposition 8 below.

Definition 19. Let $G$ be a real group, and $Z$ its center. Write

$$
\text { ad }: G(\mathbb{R}) \rightarrow G(\mathbb{R}) / Z(\mathbb{R})
$$

for the quotient map.

Note that $\operatorname{ad}(G(\mathbb{R}))$ has finite index in $G_{\mathrm{ad}}(\mathbb{R})$.

Lemma 1. For this lemma, let $G$ be a Zariski-connected reductive real group, and $K_{\mathbb{R}}$ a maximal compact subgroup of $G(\mathbb{R})$. Let $L_{\mathbb{R}}$ be a maximal compact subgroup of $G_{\mathrm{ad}}(\mathbb{R})$ containing $\operatorname{ad}\left(K_{\mathbb{R}}\right)$. Then the following hold:

(i) $K_{\mathbb{R}}$ meets all the connected components of $G(\mathbb{R})$.

(ii) $K_{\mathbb{R}} \cap G(\mathbb{R})^{+}=K_{\mathbb{R}}^{+}$.

(iii) $\operatorname{ad}\left(K_{\mathbb{B}}\right)$ is a maximal compact subgroup of $\operatorname{ad}(G(\mathbb{R}))$.

(iv) $\operatorname{ad}\left(K_{\mathbb{R}}^{+}\right)=L_{\mathbb{R}}^{+}$.

(v) $K_{\mathbb{R}} Z(\mathbb{R}) \cap G(\mathbb{R})_{+}=K_{\mathbb{R}}^{+} Z(\mathbb{R})$.

Proof. The first two statements follow from the Cartan decomposition [Satake 1980, Corollary 4.5].

For (iii), suppose that $C$ is a subgroup of $G(\mathbb{R})$ with $\operatorname{ad}\left(K_{\mathbb{R}}\right) \subseteq \operatorname{ad}(C)$ and $\operatorname{ad}(C)$ compact. If $\operatorname{ad}\left(K_{\mathbb{R}}\right) \neq \operatorname{ad}(C)$, there is an element $a \in C Z(\mathbb{R})-K_{\mathbb{R}} Z(\mathbb{R})$. By the Cartan decomposition, we may assume that $a=\exp (H)$, with $H$ a semisimple element of $\operatorname{Lie}(G)$, and $\alpha(H)$ real and nonnegative for every root $\alpha$ of $G$. Since $a \notin Z(\mathbb{R})$, we have $\alpha(H)>0$ for some root $\alpha$. Thus $\operatorname{ad}(C)$ is not compact, a contradiction. Thus $\operatorname{ad}\left(K_{\mathbb{R}}\right)=\operatorname{ad}(C)$, and statement (iii) follows.

For (iv), note that $L_{\mathbb{R}} \cap \operatorname{ad}(G)=\operatorname{ad}\left(K_{\mathbb{R}}\right)$, and therefore $L_{\mathbb{R}} / \operatorname{ad}\left(K_{\mathbb{R}}\right)$ injects into $G_{\text {ad }}(\mathbb{R}) / \operatorname{ad}(G(\mathbb{R}))$. It follows that $\operatorname{ad}\left(K_{\mathbb{R}}^{+}\right)$has finite index in $L_{\mathbb{R}}$. Since it is connected, statement (iv) follows.

For (v), let $g \in K_{\mathbb{R}} Z(\mathbb{R}) \cap G(\mathbb{R})_{+}$. Then $\operatorname{ad}(g) \in L_{\mathbb{R}} \cap G_{\mathrm{ad}}(\mathbb{R})^{+}$, so by statement (ii), we see $\operatorname{ad}(g) \in L_{\mathbb{R}}^{+}=\operatorname{ad}\left(K_{\mathbb{R}}^{+}\right)$. Thus $g \in K_{\mathbb{R}}^{+} Z(\mathbb{R})$. The other inclusion is obvious.

\section{Proposition 8.}

(i) The natural projection $p_{X}: X \rightarrow \bar{X}$ has fibers of order $\left[G(\mathbb{R}): G(\mathbb{R})_{+}\right]$. 
(ii) Let $X^{+}$be a connected component of $X$. It is stabilized by $G(\mathbb{R})_{+}$, and the restriction of $p_{X}$ to $X^{+}$is a $G(\mathbb{R})_{+}$-isomorphism onto $\bar{X}$.

(iii) Let $K$ be small. Then the natural projection $p_{S}: S_{K} \rightarrow \bar{S}_{K}$ has fibers of order $\left[G(\mathbb{R}): G(\mathbb{R})_{+}\right]$.

Proof. Consider the natural map

$$
K_{\mathbb{R}} Z(\mathbb{R}) / K_{\mathbb{R}}^{+} Z(\mathbb{R}) \rightarrow G(\mathbb{R}) / G(\mathbb{R})_{+} .
$$

It is surjective because $K_{\mathbb{R}}$ meets every connected component of $G(\mathbb{R})$. It is injective because $K_{\mathbb{R}} Z(\mathbb{R}) \cap G(\mathbb{R})_{+} \subseteq K_{\mathbb{R}}^{+} Z(\mathbb{R})$. It follows that (6-3) is an isomorphism, and the first statement follows.

We now prove the second statement. Note that $p_{X}$ is both an open and closed map, so that $p_{X}\left(X^{+}\right)$is a component of $\bar{X}$. Since $K_{\mathbb{R}}$ meets every connected component of $G(\mathbb{R})$, the set $\bar{X}$ is connected. Therefore $p_{X}\left(X^{+}\right)=\bar{X}$. By [Milne 2005, Proposition 5.7], there are $\left[G(\mathbb{R}): G(\mathbb{R})_{+}\right]$connected components of $X$, each stabilized by $G(\mathbb{R})_{+}$. Thus the fiber over a point in $\bar{X}$ is composed of exactly one point from each component of $X$. So $p_{X}$ restricted to $X^{+}$is an isomorphism; it is clear that it respects the $G(\mathbb{R})_{+}$-action.

To prove the third statement, we require $K$ to be sufficiently small, in the following way. Suppose $K_{*}$ is an open compact subgroup of $G\left(\mathbb{A}_{f}\right)$ satisfying $K_{*} \cap G(\mathbb{Q}) \subseteq G(\mathbb{Q})^{+}$. Let $g_{1}, \ldots, g_{r}$ be representatives of the finite quotient group $G(\mathbb{Q}) K_{*} \backslash G\left(\mathbb{A}_{f}\right)$. Then we require that

$$
K \subseteq \bigcap_{i=1}^{r} g_{i}^{-1} K_{*} g_{i} .
$$

Now for $x \in X$, let $\operatorname{Fib}(x)$ be the fiber of $p_{X}$ containing $x$. If we further fix $g \in G\left(\mathbb{A}_{f}\right)$, let $\operatorname{Fib}(x, g)$ be the fiber of $p_{S}$ containing $(x, g)$. (Here we understand $(x, g)$ as an element of $S_{K}$.) We claim that for all such $x$ and $g$, the map

$$
\operatorname{Fib}(x) \rightarrow \operatorname{Fib}(x, g)
$$

given by $x^{\prime} \mapsto\left(x^{\prime}, g\right)$ is a bijection. This will imply the third statement.

For surjectivity of (6-5), pick $\left(x^{\prime}, g^{\prime}\right) \in \operatorname{Fib}(x, g)$. Then there are $q \in G(\mathbb{Q})$ and $k \in G\left(\mathbb{A}_{f}\right)$ such that $q p_{X}\left(x^{\prime}\right)=p_{X}(x)$ and $q g^{\prime} k=g$. Let $x^{\prime \prime}=q x^{\prime}$. Then $x^{\prime \prime} \in \operatorname{Fib}(x)$ and $\left(x^{\prime \prime}, g\right)=\left(x^{\prime}, g^{\prime}\right)$.

For injectivity of (6-5), suppose that $\left(x_{1}, g\right)=\left(x_{2}, g\right)$ in $S_{K}$ with $x_{1}, x_{2} \in \operatorname{Fib}(x)$. Then in particular, there is an element $q \in G(\mathbb{Q})$ and $k \in K$ such that $q g k=g$ and $q x_{1}=x_{2}$. Write $g=q_{0} k_{0} g_{i}$ with $q_{0} \in G(\mathbb{Q})$ and $k_{0} \in K_{*}$. Then we have

$$
q\left(q_{0} k_{0} g_{i}\right) k=q_{0} k_{0} g_{i},
$$


which we rewrite as

$$
q_{0}^{-1} q q_{0}=k_{0} g_{i} k^{-1} g_{i}^{-1} k_{0}^{-1} .
$$

Using this and (6-4) we see that $q_{0}^{-1} q q_{0} \in G(\mathbb{Q}) \cap K_{*} \subseteq G(\mathbb{Q})^{+}$. Since $G(\mathbb{Q})^{+}$is normal in $G(\mathbb{Q})$, in fact $q \in G(\mathbb{Q})^{+}$.

Meanwhile, pick $\xi_{1}, \xi_{2} \in G(\mathbb{R})$ representing $x_{1}$ and $x_{2}$, respectively. Since $x_{1}, x_{2} \in \operatorname{Fib}(x)$ we have $\xi_{1}^{-1} \xi_{2} \in K_{\mathbb{R}} Z(\mathbb{R})$. Write $\xi_{2}=\xi_{1} k z$, with $k \in K_{\mathbb{R}}$ and $z \in Z(\mathbb{R})$. Since $q x_{1}=x_{2}$, we have $\xi_{2}^{-1} q \xi_{1} \in K_{\mathbb{R}}^{+} Z(\mathbb{R})$, and thus $z^{-1} k^{-1} \xi_{1}^{-1} q \xi_{1} \in$ $K_{\mathbb{R}}^{+} Z(\mathbb{R})$. Using the fact that $q$ is in the normal subgroup $G(\mathbb{R})_{+}$of $G(\mathbb{R})$, it follows that $k \in G(\mathbb{R})_{+} \cap K_{\mathbb{R}} \subseteq K_{\mathbb{R}}^{+} Z(\mathbb{R})$. Thus $x_{1}=x_{2}$, as desired.

Proposition 9 (Harder; see [Harder 1971; Serre 1971]). If $G$ is semisimple and $K$ is small, then $\chi_{\text {top }}\left(\Gamma_{K} \backslash \bar{X}\right)=\chi_{\text {alg }}\left(\Gamma_{K}\right)$.

Proposition 10 [Arthur 1989; Goresky et al. 1997]. If $K$ is small, then we have $\chi_{K}(G)=\chi_{\text {top }}\left(\bar{S}_{K}\right)$.

6.3. Computations. The next three lemmas will allow us to convert our computation for $K_{0}$ to a computation for $K$.

Lemma 2. If $K$ is small, then

$\left|C(\mathbb{Q})^{\dagger} \backslash C\left(\mathbb{A}_{f}\right) / v(K)\right|$

$$
=\left[v\left(K_{0}\right): v(K)\right]\left|v\left(K_{0}\right) \cap C(\mathbb{Q})^{\dagger}\right|^{-1}\left|C(\mathbb{Q})^{\dagger} \backslash C\left(\mathbb{A}_{f}\right) / v\left(K_{0}\right)\right| .
$$

Proof. This follows from the exactness of the sequence

$$
\begin{aligned}
& 1 \rightarrow v\left(K_{0}\right) \cap C(\mathbb{Q})^{\dagger} \rightarrow v\left(K_{0}\right) / v(K) \rightarrow C(\mathbb{Q})^{\dagger} \backslash C\left(\mathbb{A}_{f}\right) / v(K) \\
& \rightarrow C(\mathbb{Q})^{\dagger} \backslash C\left(\mathbb{A}_{f}\right) / v\left(K_{0}\right) \rightarrow 1 .
\end{aligned}
$$

Lemma 3. If $K \subseteq K_{0}$ is small, then

$$
\left[\Gamma_{K_{0}}^{\mathrm{ad}}: \Gamma_{K}^{\mathrm{ad}}\right]=\frac{\left[\Gamma_{K_{0}}: \rho\left(\Gamma_{K_{0}}^{\mathrm{sc}}\right)\right]\left[K_{0}: K\right]}{\left|K_{0} \cap Z(\mathbb{Q})\right|\left[v\left(K_{0}\right): v(K)\right]\left[K_{0}^{\mathrm{der}}: K^{\operatorname{der}} \rho\left(K_{0}^{\mathrm{sc}}\right)\right]} .
$$

In the proof we refer to conditions of Proposition 7.

Proof. Consider the map $\Gamma_{K_{0}}^{\mathrm{der}} / \Gamma_{K}^{\mathrm{der}} \rightarrow \Gamma_{K_{0}}^{\mathrm{ad}} / \Gamma_{K}^{\mathrm{ad}}$.

The kernel of this map sits in the middle of the exact sequence

$$
\begin{aligned}
1 \rightarrow \Gamma_{K_{0}}^{\mathrm{der}} \cap Z(\mathbb{Q}) \rightarrow\left(\Gamma_{K} Z(\mathbb{Q}) \cap\right. & \left.\Gamma_{K_{0}}^{\mathrm{der}}\right) / \Gamma_{K}^{\mathrm{der}} \\
& \rightarrow\left(\Gamma_{K} Z(\mathbb{Q}) \cap \Gamma_{K_{0}}^{\mathrm{der}}\right) / \Gamma_{K}^{\mathrm{der}}\left(\Gamma_{K_{0}}^{\mathrm{der}} \cap Z(\mathbb{Q})\right) \rightarrow 1,
\end{aligned}
$$

using condition (i). This last quotient is trivial, because actually $\Gamma_{K}=\Gamma_{K}^{\mathrm{der}}$ by condition (iii). 
We have established the exactness of the sequence

$$
1 \rightarrow \Gamma_{K_{0}}^{\mathrm{der}} \cap Z(\mathbb{Q}) \rightarrow \Gamma_{K_{0}}^{\mathrm{der}} / \Gamma_{K}^{\mathrm{der}} \rightarrow \Gamma_{K_{0}}^{\mathrm{ad}} / \Gamma_{K}^{\mathrm{ad}} \rightarrow \Gamma_{K_{0}} Z(\mathbb{Q}) / \Gamma_{K_{0}}^{\mathrm{der}} Z(\mathbb{Q}) \rightarrow 1 .
$$

The last quotient is isomorphic to $\Gamma_{K_{0}} /\left(Z(\mathbb{Q}) \cap K_{0}\right) \Gamma_{K_{0}}^{\text {der }}$, which itself sits inside the exact sequence

$$
1 \rightarrow K_{0} \cap Z(\mathbb{Q}) / \Gamma_{K_{0}}^{\mathrm{der}} \cap Z(\mathbb{Q}) \rightarrow \Gamma_{K_{0}} / \Gamma_{K_{0}}^{\mathrm{der}} \rightarrow \Gamma_{K_{0}} /\left(Z(\mathbb{Q}) \cap K_{0}\right) \Gamma_{K_{0}}^{\mathrm{der}} \rightarrow 1 .
$$

The quantity $\left|\Gamma_{K_{0}}^{\text {der }} \cap Z(\mathbb{Q})\right|$ cancels, and it follows that

$$
\left[\Gamma_{K_{0}}^{\mathrm{ad}}: \Gamma_{K}^{\mathrm{ad}}\right]=\frac{\left[\Gamma_{K_{0}}^{\mathrm{der}}: \Gamma_{K}^{\mathrm{der}}\right] \cdot\left[\Gamma_{K_{0}}: \Gamma_{K_{0}}^{\mathrm{der}}\right]}{\left|K_{0} \cap Z(\mathbb{Q})\right|} .
$$

By condition (v) we have

$$
1 \rightarrow \rho\left(\Gamma_{K_{0}}^{\mathrm{sc}}\right) / \rho\left(\Gamma_{K}^{\mathrm{sc}}\right) \rightarrow \Gamma_{K_{0}}^{\mathrm{der}} / \Gamma_{K}^{\mathrm{der}} \rightarrow \Gamma_{K_{0}}^{\mathrm{der}} / \rho\left(\Gamma_{K_{0}}^{\mathrm{sc}}\right) \rightarrow 1 .
$$

Strong approximation tells us that $G_{\mathrm{sc}}(\mathbb{Q})$ is dense in $G_{\mathrm{sc}}\left(\mathbb{A}_{f}\right)$. Therefore we have isomorphisms

$$
\rho\left(\Gamma_{K_{0}}^{\mathrm{sc}}\right) / \rho\left(\Gamma_{K}^{\mathrm{sc}}\right) \stackrel{\sim}{\rightarrow} \Gamma_{K_{0}}^{\mathrm{sc}} / \Gamma_{K}^{\mathrm{sc}} \stackrel{\sim}{\rightarrow} K_{0}^{\mathrm{sc}} / K^{\mathrm{sc}} \stackrel{\sim}{\rightarrow} \rho\left(K_{0}^{\mathrm{sc}}\right) / \rho\left(K^{\mathrm{sc}}\right) .
$$

Combining this with the exact sequences

$$
1 \rightarrow K_{0}^{\text {der }} / K^{\text {der }} \rightarrow K_{0} / K \rightarrow v\left(K_{0}\right) / v(K) \rightarrow 1
$$

and

$$
1 \rightarrow \rho\left(K_{0}^{\mathrm{sc}}\right) / \rho\left(K^{\mathrm{sc}}\right) \rightarrow K_{0}^{\mathrm{der}} / K^{\mathrm{der}} \rightarrow K_{0}^{\mathrm{der}} / K^{\mathrm{der}} \rho\left(K_{0}^{\mathrm{sc}}\right) \rightarrow 1,
$$

we obtain

$$
\left[\Gamma_{K_{0}}^{\mathrm{der}}: \Gamma_{K}^{\mathrm{der}}\right]=\frac{\left[\Gamma_{K_{0}}^{\mathrm{der}}: \rho\left(\Gamma_{K_{0}}^{\mathrm{sc}}\right)\right]\left[K_{0}: K\right]}{\left[K_{0}^{\mathrm{der}}: K^{\operatorname{der}} \rho\left(K_{0}^{\mathrm{sc}}\right)\right]\left[v\left(K_{0}\right): v(K)\right]} .
$$

Plugging this into (6-7) gives the lemma.

Corollary 1. Suppose that $K \subseteq K_{0}$ is small, and $g \in G\left(\mathbb{A}_{f}\right)$ with $g K^{-1} \subseteq K_{0}$ also small. Then

$$
\left[\Gamma_{K_{0}}^{\mathrm{ad}}: \Gamma_{g K^{-1}}^{\mathrm{ad}}\right]=\left[\Gamma_{K_{0}}^{\mathrm{ad}}: \Gamma_{K}^{\mathrm{ad}}\right] .
$$

Proof. We show that the expression (6-6) does not change when $K$ is replaced with $g K g^{-1}$. Clearly $v(K)=v\left(g K g^{-1}\right)$. Since

$$
\left[K_{0}: K\right]=\operatorname{vol}_{d g_{f}}\left(K_{0}\right) / \operatorname{vol}_{d g_{f}}(K),
$$

we have $\left[K_{0}: g K^{-1}\right]=\left[K_{0}: K\right]$. Finally, we claim that

$$
\left[K_{0}^{\mathrm{der}}:\left(g K g^{-1}\right)^{\mathrm{der}} \rho\left(K_{0}^{\mathrm{sc}}\right)\right]=\left[K_{0}^{\mathrm{der}}: K^{\mathrm{der}} \rho\left(K_{0}^{\mathrm{sc}}\right)\right] .
$$


From the exact sequence (6-8), it is enough to show that $\left[K_{0}^{\mathrm{der}}:\left(g \mathrm{Kg}^{-1}\right)^{\mathrm{der}}\right]=$ $\left[K_{0}^{\mathrm{der}}: K^{\mathrm{der}}\right]$ and $\left[\rho\left(K_{0}^{\mathrm{sc}}\right): \rho\left(\left(g K g^{-1}\right)^{\mathrm{sc}}\right)\right]=\left[\rho\left(K_{0}^{\mathrm{sc}}\right): \rho\left(K^{\mathrm{sc}}\right)\right]$. These hold because $\left(g K g^{-1}\right)^{\mathrm{der}}=g K^{\mathrm{der}} g^{-1}$ and $\rho\left(\left(g K g^{-1}\right)^{\mathrm{sc}}\right)=g \rho\left(K^{\mathrm{sc}}\right) g^{-1}$.

Lemma 4. If $G$ is semisimple and $K$ is small, then

$$
\left|\pi_{0}\left(S_{K}\right)\right|=\left[K_{0}: K \rho\left(K_{0}^{\mathrm{sc}}\right)\right]\left[\Gamma_{K_{0}}: G(\mathbb{Q})_{+} \cap \rho\left(K_{0}^{\mathrm{sc}}\right)\right]\left|\pi_{0}\left(S_{K_{0}}\right)\right| .
$$

Proof. The kernel of the projection $\pi_{0}\left(S_{K}\right) \rightarrow \pi_{0}\left(S_{K_{0}}\right)$ is isomorphic to

$$
K_{0} /\left(K G(\mathbb{Q})_{+} \cap K_{0}\right) .
$$

By [Deligne 1979, Section 2.1.3], we have $\rho\left(G_{\mathrm{sc}}\left(\mathbb{A}_{f}\right)\right) \subseteq K G(\mathbb{Q})_{+}$. Using the exact sequence

$$
1 \rightarrow\left(K_{0} \cap K G(\mathbb{Q})_{+}\right) / K \rho\left(K_{0}^{\mathrm{sc}}\right) \rightarrow K_{0} / K \rho\left(K_{0}^{\mathrm{sc}}\right) \rightarrow K_{0} /\left(K G(\mathbb{Q})_{+} \cap K_{0}\right) \rightarrow 1,
$$

we are reduced to computing the order of

$$
\left(K_{0} \cap K G(\mathbb{Q})_{+}\right) / K \rho\left(K_{0}^{\mathrm{sc}}\right) \stackrel{\sim}{\rightarrow} \Gamma_{K_{0}} /\left(K \rho\left(K_{0}^{\mathrm{sc}}\right) \cap G(\mathbb{Q})_{+}\right) .
$$

This group sits in the sequence

$$
\begin{aligned}
1 \rightarrow\left(G(\mathbb{Q})_{+} \cap K \rho\left(K_{0}^{\mathrm{sc}}\right)\right) /\left(G(\mathbb{Q})_{+} \cap \rho\left(K_{0}^{\mathrm{sc}}\right)\right) & \\
& \rightarrow \Gamma_{K_{0}} /\left(G(\mathbb{Q})_{+} \cap \rho\left(K_{0}^{\mathrm{sc}}\right)\right) \rightarrow \Gamma_{K_{0}} /\left(K \rho\left(K_{0}^{\mathrm{sc}}\right) \cap G(\mathbb{Q})_{+}\right) \rightarrow 1 .
\end{aligned}
$$

We claim the kernel is trivial. Note that $K \rho\left(K_{0}^{\mathrm{sc}}\right) \subseteq K \rho\left(G_{\mathrm{sc}}(\mathbb{Q}) K^{\mathrm{sc}}\right)$ by strong approximation. So

$$
\begin{aligned}
G(\mathbb{Q})_{+} \cap K \rho\left(K_{0}^{\mathrm{sc}}\right) & \subseteq G(\mathbb{Q})_{+} \cap K \rho\left(G_{\mathrm{sc}}(\mathbb{Q})\right) \\
& =G(\mathbb{Q})_{+} \cap(K \cap G(\mathbb{Q})) \rho\left(G_{\mathrm{sc}}(\mathbb{Q})\right) .
\end{aligned}
$$

Since $K \cap G(\mathbb{Q}) \subseteq \rho\left(G_{\text {sc }}(\mathbb{Q})\right)$ by Proposition 7(v), we have $G(\mathbb{Q})_{+} \cap K \rho\left(K_{0}^{\text {sc }}\right) \subseteq$ $G(\mathbb{Q})_{+} \cap \rho\left(K_{0}^{\mathrm{sc}}\right)$. This proves the claim, and the lemma follows.

In the course of proving the theorem, we will pass to the adjoint group to apply Harder's theorem (Proposition 9), but lift to $G_{\mathrm{sc}}$ to apply Harder's calculation (Proposition 6). We must record the difference between Serre's Euler characteristic at $G_{\text {ad }}$ and $G_{\text {sc }}$.

Lemma 5. We have

$$
\chi_{\text {alg }}\left(\Gamma_{K_{0}}^{\mathrm{ad}}\right)=\frac{|\operatorname{ker}(\rho(\mathbb{Q}))|\left|K_{0} \cap Z(\mathbb{Q})\right|}{\left[\Gamma_{K_{0}}^{\mathrm{der}}: \rho\left(\Gamma_{K_{0}}^{\mathrm{sc}}\right)\right]\left[\Gamma_{K_{0}}: \Gamma_{K_{0}}^{\mathrm{der}}\right]} \chi_{\mathrm{alg}}\left(\Gamma_{K_{0}}^{\mathrm{sc}}\right) .
$$

Proof. This follows from the properties of $\chi_{\text {alg }}$ mentioned earlier. 
Proof of Theorem 2. Pick a set $g_{1}, \ldots, g_{r}$ of representatives of $\pi_{0}\left(S_{K_{0}}\right)$, viewed as a quotient of $G\left(\mathbb{A}_{f}\right)$ as in (6-1).

Let $K$ be small subgroup of finite index in $K_{0}$. Possibly by intersecting finitely many conjugates of $K$, we may assume that

- $K$ is normal in $K_{0}$ and

- $g_{i} K g_{i}^{-1}$ is a small subgroup of $K_{0}$ for all $i$.

By Proposition 10, $\chi_{K}(G)=\chi_{\text {top }}\left(\bar{S}_{K}\right)$. By Proposition 8 , this is equal to $\left[G(\mathbb{R}): G(\mathbb{R})_{+}\right]^{-1} \chi_{\text {top }}\left(S_{K}\right)$. Write $\Gamma_{g}$ for $\Gamma_{g K g^{-1}}^{\text {ad }}$. By [Deligne 1979, 2.1.2], the components of $S_{K}$ are each isomorphic to $\Gamma_{g} \backslash X^{+}$, where $X^{+}$is a component of $X$. Here $g$ runs over $\pi_{0}\left(S_{K}\right)$.

By Proposition 8, the topological spaces $\Gamma_{g} \backslash X^{+}$and $\Gamma_{g} \backslash \bar{X}$ are isomorphic. Therefore we have $\chi_{\text {top }}\left(\Gamma_{g} \backslash X^{+}\right)=\chi_{\text {top }}\left(\Gamma_{g} \backslash \bar{X}\right)$.

Applying Proposition 9 to $G_{\mathrm{ad}}$, this is equal to $\chi_{\mathrm{alg}}\left(\Gamma_{g}\right)$. Therefore

$$
\chi_{K}(G)=\left[G(\mathbb{R}): G(\mathbb{R})_{+}\right]^{-1} \sum_{g \in \pi_{0}\left(S_{K}\right)} \chi_{\mathrm{alg}}\left(\Gamma_{g}\right) .
$$

Every element in $\pi_{0}\left(S_{K}\right)$ may be written as the product of an element of $\pi_{0}\left(S_{K_{0}}\right)$ with an element of $K_{0}$. Since $K$ is normal in $K_{0}$, the groups $\Gamma_{g k_{0}}$ and $\Gamma_{g}$ are equal for $k_{0} \in K_{0}$. It follows that

$$
\chi_{K}(G)=\frac{\left|\pi_{0}\left(S_{K}\right)\right|}{\left[G(\mathbb{R}): G(\mathbb{R})_{+}\right]\left|\pi_{0}\left(S_{K_{0}}\right)\right|} \sum_{i=1}^{r} \chi_{\mathrm{alg}}\left(\Gamma_{g_{i}}\right) .
$$

By Corollary 1 we have

$$
\chi_{\text {alg }}\left(\Gamma_{g_{i}}\right)=\left[\Gamma_{K_{0}}^{\mathrm{ad}}: \Gamma_{g_{i}}\right] \chi_{\mathrm{alg}}\left(\Gamma_{K_{0}}^{\mathrm{ad}}\right)=\left[\Gamma_{K_{0}}^{\mathrm{ad}}: \Gamma_{K}^{\mathrm{ad}}\right] \chi_{\mathrm{alg}}\left(\Gamma_{K_{0}}^{\mathrm{ad}}\right) .
$$

This gives

$$
\chi_{K}(G)=\left[G(\mathbb{R}): G(\mathbb{R})_{+}\right]^{-1}\left[\Gamma_{K_{0}}^{\mathrm{ad}}: \Gamma_{K}^{\mathrm{ad}}\right]\left|\pi_{0}\left(S_{K}\right)\right| \chi_{\mathrm{alg}}\left(\Gamma_{K_{0}}^{\mathrm{ad}}\right) .
$$

The component group $\pi_{0}\left(S_{K}\right)$ fits into the exact sequence

$$
1 \rightarrow G_{\mathrm{der}}\left(\mathbb{A}_{f}\right) /\left(G_{\mathrm{der}}\left(\mathbb{A}_{f}\right) \cap G(\mathbb{Q})_{+} K\right) \rightarrow \pi_{0}\left(S_{K}\right) \rightarrow C(\mathbb{Q})^{\dagger} \backslash C\left(\mathbb{A}_{f}\right) / v(K) \rightarrow 1
$$

This gives

$$
\chi_{K}(G)=\left[G(\mathbb{R}): G(\mathbb{R})_{+}\right]^{-1}\left|\pi_{0}\left(S_{K}^{\text {der }}\right)\right|\left|C(\mathbb{Q})^{\dagger} \backslash C\left(\mathbb{A}_{f}\right) / \nu(K)\right|\left[\Gamma_{K_{0}}^{\mathrm{ad}}: \Gamma_{K}^{\mathrm{ad}}\right] \chi_{\mathrm{alg}}\left(\Gamma_{K_{0}}^{\mathrm{ad}}\right) .
$$

where here $\pi_{0}\left(S_{K^{\mathrm{der}}}\right)=G_{\mathrm{der}}\left(\mathbb{A}_{f}\right) / G_{\mathrm{der}}\left(\mathbb{Q}_{+}\right)^{\mathrm{der}}$.

Using $\chi_{K_{0}}(G)=\left[K_{0}: K\right]^{-1} \chi_{K}(G)$ together with Lemma 2 gives

$\chi_{K_{0}}(G)=\frac{\left|\pi_{0}\left(S_{K^{\mathrm{der}}}\right)\right|\left[v\left(K_{0}\right): v(K)\right]\left|C(\mathbb{Q})^{\dagger} \backslash C\left(\mathbb{A}_{f}\right) / v\left(K_{0}\right)\right|\left[\Gamma_{K_{0}}^{\mathrm{ad}}: \Gamma_{K}^{\mathrm{ad}}\right]}{\left[G(\mathbb{R}): G(\mathbb{R})_{+}\right]\left|v\left(K_{0}\right) \cap C(\mathbb{Q})^{\dagger}\right|\left[K_{0}: K\right]} \chi_{\mathrm{alg}}\left(\Gamma_{K_{0}}^{\mathrm{ad}}\right)$. 
By Lemmas 3 and 5,

$$
\chi_{K_{0}}(G)=\frac{|\operatorname{ker}(\rho(\mathbb{Q}))|\left|\pi_{0}\left(S_{K^{\text {der }}}\right)\right|\left|C(\mathbb{Q})^{\dagger} \backslash C\left(\mathbb{A}_{f}\right) / v\left(K_{0}\right)\right|}{\left[G(\mathbb{R}): G(\mathbb{R})_{+}\right]\left|v\left(K_{0}\right) \cap C(\mathbb{Q})^{\dagger}\right|\left[K_{0}^{\text {der }}: K^{\operatorname{der}} \rho\left(K_{0}^{\text {sc }}\right)\right]} \chi_{\mathrm{alg}}\left(\Gamma_{K_{0}}^{\text {sc }}\right) .
$$

The theorem then follows from Lemma 4.

6.4. Examples. We now use Theorem 2 and Proposition 6 to explicitly compute some cases of $\chi_{K_{0}}(G)$. Recall that we write $\mathscr{O}_{f}$ for the integer points of $\mathbb{A}_{f}$.

Corollary 2. If $T$ is a torus and $K_{0} \subset T\left(\mathbb{A}_{f}\right)$ is a compact open subgroup, then

$$
\chi_{K_{0}}(T)=\left|T(\mathbb{Q}) \backslash T\left(\mathbb{A}_{f}\right) / K_{0}\right| \cdot\left|K_{0} \cap T(\mathbb{Q})\right|^{-1} .
$$

Let $T=\mathbb{G}_{m}$, and $K_{0}=T\left(\mathscr{O}_{f}\right)$. Then $\chi_{K_{0}}(T)=1 / 2$.

Let $T$ be the norm-one subgroup of an imaginary quadratic extension $E$ of $\mathbb{Q}$. Let $K_{0}=T\left(\mathscr{O}_{f}\right)$. Write $\mathscr{O}(E)$ for the integer points of the adeles $\mathbb{A}_{E}$ over $E$. Then $T(\mathbb{Q}) \backslash T\left(\mathbb{A}_{f}\right) / K_{0}$ injects into $E^{\times} \backslash \mathbb{A}_{E, f}^{\times} / \mathcal{O}(E)^{\times}$, which is in bijection with the class group. If the class number of $E$ is trivial, it follows that $\chi_{K_{0}}(T)=|T(\mathbb{Z})|^{-1}$.

Corollary 3. If $G$ is semisimple and simply connected, then

$$
\chi_{K_{0}}(G)=\left[G(\mathbb{R}): G(\mathbb{R})_{+}\right]^{-1} \chi_{\mathrm{alg}}\left(\Gamma_{K_{0}}\right) .
$$

Let $G=\mathrm{SL}_{2}$ and $K_{0}=G\left(0_{f}\right)$. Then

$$
\chi_{K_{0}}(G)=\chi_{\mathrm{alg}}\left(\mathrm{SL}_{2}(\mathbb{Z})\right)=-\frac{1}{2} B_{2}=-2^{-2} 3^{-1} .
$$

Let $G=\mathrm{Sp}_{4}$ and $K_{0}=G\left(0_{f}\right)$. Then

$$
\chi_{K_{0}}(G)=\chi_{\mathrm{alg}}\left(\operatorname{Sp}_{4}(\mathbb{Z})\right)=-\frac{1}{8} B_{2} B_{4}=-2^{-5} 3^{-2} 5^{-1} .
$$

When the derived group is simply connected the calculation is not much harder.

Corollary 4. If $G_{\mathrm{der}}$ is simply connected, then

$$
\chi_{K_{0}}(G)=\frac{\left|C(\mathbb{Q})^{\dagger} \backslash C\left(\mathbb{A}_{f}\right) / v\left(K_{0}\right)\right|}{\left[G(\mathbb{R}): G(\mathbb{R})_{+}\right]\left|v\left(K_{0}\right) \cap C(\mathbb{Q})^{\dagger}\right|} \chi_{\mathrm{alg}}\left(\Gamma_{K_{0}}^{\mathrm{der}}\right) .
$$

Let $G=\mathrm{GL}_{2}$ and $K_{0}=G\left(\mathrm{O}_{f}\right)$. Then $\chi_{K_{0}}(G)=\frac{1}{2} \chi_{\mathrm{alg}}\left(\mathrm{SL}_{2}(\mathbb{Z})\right)=-2^{-3} 3^{-1}$.

Let $G=\mathrm{GSp}_{4}$ and $K_{0}=G\left(\mathcal{O}_{f}\right)$. Then $\chi_{K_{0}}(G)=\frac{1}{2} \chi_{\mathrm{alg}}\left(\operatorname{Sp}_{4}(\mathbb{Z})\right)=-2^{-6} 3^{-2} 5^{-1}$.

Lemma 6. If all the points of $\operatorname{ker} \rho$ are $\mathbb{Q}$-rational, then

$$
\left[\Gamma_{K_{0}}^{\mathrm{der}}: G_{\mathrm{der}}(\mathbb{Q})_{+} \cap \rho\left(K_{0}^{\mathrm{sc}}\right)\right]=1 .
$$

Proof. By [Deligne 1979, Section 2.0.3], we have an injection

$$
G_{\mathrm{der}}(\mathbb{Q}) / \rho\left(G_{\mathrm{sc}}(\mathbb{Q})\right) \hookrightarrow H^{1}(\operatorname{im}(\operatorname{Gal}(\overline{\mathbb{Q}} / \mathbb{Q})),(\operatorname{ker} \rho)(\overline{\mathbb{Q}})),
$$


using the cohomology group defined in that paper. We also have an injection

$$
\Gamma_{K_{0}}^{\mathrm{der}} /\left(G_{\mathrm{der}}(\mathbb{Q})_{+} \cap \rho\left(K_{0}^{\mathrm{sc}}\right)\right) \hookrightarrow G_{\mathrm{der}}(\mathbb{Q}) / \rho\left(G_{\mathrm{sc}}(\mathbb{Q})\right) .
$$

Since all the points of $\operatorname{ker} \rho$ are $\mathbb{Q}$-rational, all these groups are trivial.

Let $G=\mathrm{PGL}_{2}$ and $K_{0}=G\left(\mathbb{O}_{f}\right)$. The only nontrivial factors in the formula are $\left[G(\mathbb{R}): G(\mathbb{R})_{+}\right]=2$, $|\operatorname{ker} \rho(\mathbb{Q})|=2$, and $\chi_{\text {alg }}\left(\mathrm{SL}_{2}(\mathbb{Z})\right)=-2^{-2} 3^{-1}$. Thus $\chi_{K_{0}}(G)=-2^{-2} 3^{-1}$.

\section{The case of $\mathrm{SL}_{2}$}

Let $G=\mathrm{SL}_{2}$, defined over $\mathbb{Q}$. Let $A$ be the subgroup of diagonal matrices in $G$, and let $T$ be the maximal elliptic torus of $G$ given by matrices

$$
\gamma_{a, b}=\left(\begin{array}{cc}
a & -b \\
b & a
\end{array}\right)
$$

with $a^{2}+b^{2}=1$.

The characters and cocharacters of $T$ are both isomorphic to $\mathbb{Z}$. We identify $\mathbb{Z} \stackrel{\sim}{\rightarrow} X^{*}(T)$ via $n \mapsto \chi_{n}$, where $\chi_{n}\left(\gamma_{a, b}\right)=(a+b i)^{n}$. We specify $\mathbb{Z} \stackrel{\sim}{\rightarrow} X_{*}(T)$ by identifying $n$ with the cocharacter taking $\alpha$ to $\operatorname{diag}\left(\alpha, \alpha^{-1}\right)$. The roots of $T$ in $G$ are then $\{ \pm 2\}$, and the coroots of $T$ in $G$ are $\{ \pm 1\}$. The Weyl group $\Omega$ of these systems has order 2 and the compact Weyl group $\Omega_{\mathbb{R}}$ is trivial. Thus each $L$-packet of discrete series has order 2 . The group dual to $G$ is $\hat{G}=\mathrm{PGL}_{2}(\mathbb{C})$ in the usual way.

Pick an element $\xi \in G(\mathbb{C})$ such that

$$
\operatorname{Ad}(\xi)\left(\begin{array}{cc}
a & -b \\
b & a
\end{array}\right)=\left(\begin{array}{ll}
a+i b & \\
& a-i b
\end{array}\right),
$$

and put $B_{T}=\operatorname{Ad}\left(\xi^{-1}\right) B_{A}$. Then $B_{T}$ is a Borel subgroup of $G(\mathbb{C})$ containing $T$.

Consider the Langlands parameter $\varphi_{G}: W_{\mathbb{R}} \rightarrow \hat{G}$ given by $\varphi_{G}(\tau)=\left(\begin{array}{ll}0 & 1 \\ 1 & 0\end{array}\right) \times 1$, and

$$
\varphi_{G}(z)=\operatorname{diag}\left(z^{n}, \bar{z}^{n}\right) \times z=z^{\mu} \bar{z}^{v} \times z,
$$

where $\mu$ corresponds to $n \in X_{*}(\hat{T}) \stackrel{\sim}{\rightarrow} X^{*}(T)$ and $v$ corresponds to $-n$. The corresponding representation $E$ of $G(\mathbb{C})$ has highest weight $\lambda_{B}=n-1 \in X^{*}(T)$. It is the $(n-1)$-st symmetric power of the standard representation. Its central character is $\lambda_{E}(z)=z^{n-1}$, where $z= \pm 1$.

We put $\pi_{G}=\pi\left(\varphi_{G}, B_{T}\right)$, in the notation from Section 2.2. Write $\pi_{G}^{\prime}$ for the other discrete series representation in $\Pi_{E}$. Thus the $L$-packet determined by $\varphi_{G}$ is

$$
\Pi_{E}=\left\{\pi_{G}, \pi_{G}^{\prime}\right\} .
$$

We will put $f_{\infty} d g_{\infty}=e_{\pi_{G}}$ as in Section 2.4. 
7.1. Main term. First we consider the terms $S T_{g}(f d g, \pm 1)$.

We have $S \Phi_{G}\left(1, e_{\pi_{G}}\right)=-n \bar{v}\left(G ; d g_{\infty}\right)^{-1}$, and so

$$
S T_{g}(f d g, \pm 1, G)=( \pm 1)^{n} n \bar{v}\left(G ; d g_{\infty}\right)^{-1} f^{\infty}( \pm 1) .
$$

We have $S \Phi_{A}\left(1, e_{\pi_{G}}\right)=-\bar{v}\left(G ; d g_{\infty}\right)^{-1}$, and so

$$
S T_{g}(f d g, \pm 1, A)=( \pm 1)^{n} \frac{1}{2} \bar{v}\left(G ; d g_{\infty}\right)^{-1} f_{A}^{\infty}( \pm 1) .
$$

If $\gamma$ is a regular semisimple element of $G(\mathbb{C})$ with eigenvalues $\alpha$ and $\alpha^{-1}$, then according to the Weyl character formula,

$$
\operatorname{tr}(\gamma ; E)=\frac{\alpha^{n}-\alpha^{-n}}{\alpha-\alpha^{-1}}
$$

Define $t_{4}(n)=\operatorname{tr}(\operatorname{diag}(i,-i) ; E)$, where $i$ is a fourth root of unity. Then $t_{4}(n)=0$ if $n$ is even, and $t_{4}(n)=(-1)^{(n-1) / 2}$ if $n$ is odd.

Similarly, define $t_{3}(n)=\operatorname{tr}\left(\operatorname{diag}\left(\zeta, \zeta^{2}\right) ; E\right)$, where $\zeta$ is a third root of unity. Then $t_{3}(n)=[0,1,-1 ; 3]_{n}$, meaning that

$$
t_{3}(n)=\left\{\begin{aligned}
0 & \text { if } n \equiv 0 \\
1 & \text { if } n \equiv 1, \\
-1 & \text { if } n \equiv 2
\end{aligned}\right.
$$

Here the congruence is modulo 3.

There are three stable conjugacy classes of elliptic $\gamma \in G(\mathbb{Q})$, which we represent by

$$
\gamma_{3}=\left(\begin{array}{rc}
-1 & -1 \\
1 & 0
\end{array}\right), \quad \gamma_{4}=\left(\begin{array}{cc}
0 & -1 \\
1 & 0
\end{array}\right), \quad \gamma_{6}=\left(\begin{array}{cr}
0 & -1 \\
1 & 1
\end{array}\right)
$$

Note that $-\gamma_{4} \sim \gamma_{4}, \gamma_{6}^{2}=\gamma_{3}$, and $-\gamma_{3} \sim \gamma_{6}$.

Write $T_{3}$ for the elliptic torus consisting of elements

$$
\left(\begin{array}{cc}
a & a-b \\
b-a & b
\end{array}\right), \quad \text { with } a^{2}-a b+b^{2}=1 .
$$

We have $S \Phi_{G}\left(\gamma_{3}, e_{\pi_{G}}\right)=-\bar{v}\left(T_{3}\right)^{-1} t_{3}(n)$, and so

$$
S T_{g}\left(f d g, \gamma_{3}, G\right)=-\bar{v}\left(T_{3}\right)^{-1} S O_{\gamma_{3}}\left(f^{\infty} d g_{f}\right) t_{3}(n) .
$$

We have $S \Phi_{G}\left(\gamma_{4}, e_{\pi_{G}}\right)=-\bar{v}(T)^{-1} t_{4}(n)$, and so

$$
S T_{g}\left(f d g, \gamma_{4}, G\right)=-\bar{v}(T)^{-1} S O_{\gamma_{4}}\left(f^{\infty} d g_{f}\right) t_{4}(n) .
$$

Finally $S \Phi_{G}\left(\gamma_{6}, e_{\pi_{G}}\right)=-\bar{v}\left(T_{3}\right) t_{3}(n)(-1)^{n-1}$, and so

$$
S T_{g}\left(f d g, \gamma_{6}, G\right)=-\bar{v}\left(T_{3}\right)^{-1} S O_{-\gamma_{3}}\left(f^{\infty} d g_{f}\right) t_{3}(n)(-1)^{n-1} .
$$


Thus, $S T_{g}(f d g)$ is equal to the sum

$$
\begin{gathered}
-n \bar{v}\left(G ; d g_{\infty}\right)^{-1} f^{\infty}(1)+n \bar{v}\left(G ; d g_{\infty}\right)^{-1} f^{\infty}(-1)(-1)^{n}-\frac{1}{2} \bar{v}\left(A ; d a_{\infty}\right)^{-1} f_{A}^{\infty}(1) \\
+\frac{1}{2} \bar{v}\left(A ; d a_{\infty}\right)^{-1} f_{A}^{\infty}(-1)(-1)^{n}-\bar{v}\left(T_{3}\right)^{-1} S O_{\gamma_{3}}\left(f^{\infty} d g_{f}\right) t_{3}(n) \\
-\bar{v}(T)^{-1} S O_{\gamma_{4}}\left(f^{\infty} d g_{f}\right) t_{4}(n)+\bar{v}\left(T_{3}\right)^{-1} S O_{-\gamma_{3}}\left(f^{\infty} d g_{f}\right) t_{3}(n)(-1)^{n} .
\end{gathered}
$$

\subsection{Endoscopic terms.}

Definition 20. Let $E$ be an imaginary quadratic extension of $\mathbb{Q}$. Write $H_{E}$ for the kernel of the norm map $\operatorname{Res}_{\mathbb{Q}}^{E} \mathbb{G}_{m} \rightarrow \mathbb{G}_{m}$.

The $H_{E}$ comprise the (proper) elliptic endoscopic groups for $G=\mathrm{SL}_{2}$. For each $H=H_{E}$ we have $\tau(H)=2$ and $|\operatorname{Out}(H, s, \eta)|=1$; see [Kottwitz 1984, Section 7]. Therefore $\iota(G, H)=\frac{1}{2}$. The character identities of Shelstad [1982] give $e_{\pi_{G}}^{H}=e_{\chi_{n}}+e_{\chi_{n}^{-1}}$.

Write $f^{H} d h=f^{\infty H} d h_{f} e_{\pi_{G}}^{H}$, where $f^{\infty H} d h_{f}$ is the transfer of $f^{\infty} d g_{f}$. Choose $d h_{\infty}$ so that $d h_{f} d h_{\infty}$ is the Tamagawa measure on $H$. Then we obtain

$$
S T_{g}\left(f^{H} d h\right)=2 \bar{v}\left(H ; d h_{\infty}\right) \sum_{\gamma_{H}} f^{\infty, H}\left(\gamma_{H}\right) \operatorname{Tr}_{\mathbb{Q}}^{E}\left(\gamma_{H}^{n}\right),
$$

the sum being taken over $\gamma_{H} \in H(\mathbb{Q})$.

Remark. Consider the local transfer, where $f_{p} d g_{p}$ is a spherical (that is, invariant under $\left.G\left(\mathbb{Z}_{p}\right)\right)$ measure on $G\left(\mathbb{Q}_{p}\right)$. Then if $H$ ramifies over $p$, a representation $\pi_{p}$ in one of the $L$-packets transferring from $H$ will also be ramified. This means that $\operatorname{tr} \pi_{p}\left(f_{p} d g_{p}\right)=0$. So we take $f_{p}^{H}=0$ in this case. Thus

$$
\mathscr{K}(f d g)=S T_{g}(f d g)
$$

there is no (proper) endoscopic contribution. This is compatible with the fact that $m_{\text {disc }}$ is constant on $L$-packets in this case.

7.3. Case of $\boldsymbol{\Gamma}=\mathbf{S L}_{2}(\mathbb{Z})$. We take $K_{f}=K_{0}$ to be the integral points of $G\left(\mathbb{A}_{f}\right)$. Also let $K_{A}=K_{0} \cap A\left(\mathbb{A}_{f}\right)$ and $K_{T}=K_{0} \cap T\left(\mathbb{A}_{f}\right)$. Each of these breaks into a product of local groups $K_{0, p}$, etc.

We put $f^{\infty} d g_{f}=e_{K_{0}}$. Note that $f^{\infty}(g)=f^{\infty}(-g)$ for all $g \in G\left(\mathbb{A}_{f}\right)$ and $f_{A}^{\infty}(a)=f_{A}^{\infty}(-a)$ for all $a \in A\left(\mathbb{A}_{f}\right)$. Therefore, if $n$ is even, then $S T_{g}(f d g)=0$. So assume henceforth that $n$ is odd. Then our expression is equal to

$$
\begin{aligned}
& -2 n \bar{v}\left(G ; d g_{\infty}\right)^{-1} f^{\infty}(1)-\bar{v}\left(A ; d a_{\infty}\right)^{-1} f_{A}^{\infty}(1) \\
& \quad-2 \bar{v}\left(T_{3}\right)^{-1} S O_{\gamma_{3}}\left(f^{\infty} d g_{f}\right) t_{3}(n)+\bar{v}(T)^{-1} S O_{\gamma_{4}}\left(f^{\infty} d g_{f}\right)(-1)^{(n+1) / 2} .
\end{aligned}
$$


We have

$$
\begin{aligned}
-2 n \bar{v}\left(G ; d g_{\infty}\right)^{-1} f^{\infty}(1) & =-2 n \bar{v}\left(G ; d g_{\infty}\right)^{-1} \operatorname{vol}_{d g_{f}}\left(K_{0}\right)^{-1} \\
& =-2 n \tau(G)^{-1} d(G)^{-1} \chi_{K_{0}}(G)=\frac{1}{12} n, \\
-\bar{v}\left(A ; d a_{\infty}\right)^{-1} f_{A}^{\infty}(1) & =-\bar{v}\left(A ; d a_{\infty}\right)^{-1} \operatorname{vol}_{d a_{f}}\left(K_{A}\right)^{-1} \\
& =-\tau(A)^{-1} d(A)^{-1} \chi_{K_{A}}(A)=-\frac{1}{2} .
\end{aligned}
$$

Now we consider $S O_{\gamma_{4}}\left(f^{\infty} d g_{f} ; d t_{f}\right)$. We have $1-\alpha\left(\gamma_{4}\right)=2$ for the positive root $\alpha$ of $G$. Therefore by Proposition 1, the local orbital integrals are equal to $\operatorname{vol}_{d t_{p}}\left(K_{T, 2}\right)^{-1}$ for $p \neq 2$. At $p=2$, one has two stable conjugacy classes $\gamma_{4}$ and $\gamma_{4}^{\prime}$ in the conjugacy class of $\gamma_{4}$, where $\gamma_{4}^{\prime}=\left(\begin{array}{cc}0 & 1 \\ -1 & 0\end{array}\right)$.

It follows that

$$
S O_{\gamma_{4}}\left(f^{\infty} d g_{f} ; d t_{f}\right)=\left(O_{\gamma_{4}}\left(e_{K_{2}} ; d t_{2}\right)+O_{\gamma_{4}^{\prime}}\left(e_{K_{2}} ; d t_{2}\right)\right) \prod_{p \neq 2} \operatorname{vol}_{d t_{p}}\left(T\left(\mathbb{Q}_{p}\right) \cap K_{p}\right)^{-1} \text {. }
$$

To compute the local integral at $p=2$, we reduce to a $\mathrm{GL}_{2}$-computation by the following lemma. Its proof is straightforward.

Lemma 7. Let $F$ be a p-adic local field with ring of integers $\mathrm{O}$. Put $G=\mathrm{SL}_{2}$, $\tilde{G}=\mathrm{GL}_{2}$, and $Z$ for the center of $\tilde{G}$. Pick Haar measures $d g$ on $G(F)$, $d \tilde{g}$ on $\tilde{G}(F)$, and $d z$ on $Z(F)$. Let $f \in C_{c}(Z(F) \backslash \tilde{G}(F))$. Then

$$
\frac{\operatorname{vol}_{d z}(Z(\mathcal{O}))}{\operatorname{vol}_{d \tilde{g}}(\tilde{G}(\mathcal{O}))} \int_{Z(F) \backslash \tilde{G}(F)} f(g) \frac{d \tilde{g}}{d z}=\operatorname{vol}_{d g}(G(\mathcal{O}))^{-1}\left|\mathcal{O}^{\times} / \mathcal{O}^{\times 2}\right|^{-1} \sum_{\alpha} \int_{G(F)} f\left(t_{\alpha} g\right) d g .
$$

Here $\alpha$ runs over the square classes in $F^{\times}$, and $t_{\alpha}=\operatorname{diag}(\alpha, 1)$.

Proposition 11. We have

$$
O_{\gamma_{4}}\left(e_{K_{2}} ; d t_{2}\right)+O_{\gamma_{4}^{\prime}}\left(e_{K_{2}} ; d t_{2}\right)=2 \operatorname{vol}_{d t_{2}}\left(K_{T, 2}\right)^{-1} \text {. }
$$

Proof. Write $\tilde{f}_{2}$ for the characteristic function of $\mathrm{GL}_{2}\left(\mathbb{Z}_{2}\right) Z\left(\mathbb{Q}_{2}\right)$. By the lemma, $\int_{Z\left(\mathbb{Q}_{2}\right) \backslash \mathrm{GL}_{2}\left(\mathbb{Q}_{2}\right)} \tilde{f}_{2}\left(g^{-1} \gamma_{4} g\right) \frac{d \tilde{g}}{d z}=\operatorname{vol}_{d t_{2}}\left(K_{T, 2}\right)\left|\mathbb{Z}_{2}^{\times} / \mathbb{Z}_{2}^{\times 2}\right|^{-1} \sum_{\alpha} O_{\mathrm{Ad}\left(t_{\alpha}\right)\left(\gamma_{4}\right)}\left(e_{K_{0}} ; d t_{2}\right)$.

Here we are normalizing $d \tilde{g}$ and $d z$ so that $\operatorname{vol}_{d z}\left(Z\left(\mathbb{Z}_{2}\right)\right)=\operatorname{vol}_{d \tilde{g}}\left(\mathrm{GL}_{2}\left(\mathbb{Z}_{2}\right)\right)=1$.

In fact, $\operatorname{Ad}\left(t_{\alpha}\right)\left(\gamma_{4}\right)$ is conjugate in $G\left(\mathbb{Q}_{2}\right)$ to $\gamma_{4}$ if and only if $\alpha$ is a norm from $\mathbb{Q}_{2}(\sqrt{-1})$, and in the contrary case, it is conjugate to $\gamma_{4}^{\prime}$. It follows that

$$
\int_{Z\left(\mathbb{Q}_{2}\right) \backslash \mathrm{GL}_{2}\left(\mathbb{Q}_{2}\right)} \tilde{f}_{2}\left(g^{-1} \gamma_{4} g\right) \frac{d \tilde{g}}{d z}=\left(O_{\gamma_{4}}\left(e_{K_{2}} ; d t_{2}\right)+O_{\gamma_{4}^{\prime}}\left(e_{K_{2}} ; d t_{2}\right)\right) \operatorname{vol}_{d t_{2}}\left(K_{T, 2}\right) .
$$

By an elliptic orbital integral computation in [Kottwitz 2005], the left hand side is equal to 2 . 
We conclude that

$$
S O_{\gamma_{4}}\left(f^{\infty} d g_{f} ; d t_{f}\right)=2 \operatorname{vol}_{d t_{f}}\left(T\left(\mathbb{A}_{f}\right) \cap K_{0}\right)^{-1},
$$

and so

$$
\begin{aligned}
-\bar{v}(T)^{-1} S O_{\gamma_{4}}\left(f^{\infty} d g_{f}\right) t_{4}(n) & =-2 \bar{v}(T)^{-1} \operatorname{vol}_{d t_{f}}\left(T\left(\mathbb{A}_{f}\right) \cap K_{0}\right)^{-1} t_{4}(n) \\
& =-2 \tau(T)^{-1} \chi_{K_{T}}(T) t_{4}(n)=2^{-2}(-1)^{(n+1) / 2} .
\end{aligned}
$$

Similarly, we find that

$$
S O_{\gamma_{3}}\left(f^{\infty} d g_{f}\right)=2 \operatorname{vol}_{d t_{3, f}}\left(T_{3}\left(\mathbb{A}_{f}\right) \cap K_{0}\right)^{-1},
$$

and so

$$
-2 \bar{v}\left(T_{3}\right)^{-1} S O_{\gamma_{3}}\left(f^{\infty} d g_{f}\right) t_{3}(n)=-3^{-1} t_{3}(n) .
$$

We conclude that in this case,

$$
S T_{g}(f d g)=\frac{1}{12} n-\frac{1}{2}+\frac{1}{4}(-1)^{(n+1) / 2}-\frac{1}{3} t_{3}(n) .
$$

Note that for $n>1$ this agrees precisely with the discrete series multiplicities. For $n=1$, this expression is equal to -1 , but of course in this case $\pi$ is not regular.

\section{Real tori}

We have finished our discussion of $\mathrm{SL}_{2}$. Starting with this section, we begin to work out the example of $\mathrm{GSp}_{4}$. Various isomorphisms of tori must be written carefully, so we begin by explicitly working out their parametrizations.

8.1. The real tori $\mathbb{G}_{m}, S$, and $T_{1}$. We identify the group of characters of $\mathbb{G}_{m}$ with $\mathbb{Z}$ in the usual way, via $\left(a \mapsto a^{n}\right) \leftrightarrow n$.

Let $A_{0}=\mathbb{G}_{m} \times \mathbb{G}_{m}$, viewed as a maximal torus in $\mathrm{GL}_{2}$ in the usual way. Via the identification above we obtain $X^{*}\left(A_{0}\right) \cong \mathbb{Z}^{2}$ and $X_{*}\left(A_{0}\right) \cong \mathbb{Z}^{2}$.

Let $S=\operatorname{Res}_{\mathbb{R}}^{\mathbb{C}} \mathbb{G}_{m}$. Recall that $\operatorname{Res}_{\mathbb{R}}^{\mathbb{C}} \mathbb{G}_{m}$ denotes the algebraic group over $\mathbb{R}$ whose $\mathscr{A}$-points are $(\mathscr{A} \otimes \mathbb{C})^{\times}$for an $\mathbb{R}$-algebra $\mathscr{A}$. By choosing the basis $\{1, i\}$ of $\mathbb{C}$ over $\mathbb{R}$, we have an injection $(\mathscr{A} \times \mathbb{C})^{\times} \rightarrow \mathrm{GL}(\mathscr{A} \otimes \mathbb{C}) \cong \mathrm{GL}_{2}(\mathscr{A})$. Thus we have an embedding $\iota_{S}: S \rightarrow \mathrm{GL}_{2}$ as an elliptic maximal torus.

There is a ring isomorphism $\varphi: \mathbb{C} \otimes \mathbb{C} \stackrel{\sim}{\rightarrow} \times \mathbb{C}$ such that $\varphi\left(z_{1} \otimes z_{2}\right)=\left(z_{1} z_{2}, z_{1} \bar{z}_{2}\right)$, which restricts to an isomorphism $\varphi: S(\mathbb{C}) \sim \mathbb{G}_{m}(\mathbb{C}) \times \mathbb{G}_{m}(\mathbb{C})$. This isomorphism is also actualized by conjugation within $\mathrm{GL}_{2}(\mathbb{C})$. Fix $x \in \mathrm{GL}_{2}(\mathbb{C})$ so that

$$
\operatorname{Ad}(x)\left(\begin{array}{cc}
a & -b \\
b & a
\end{array}\right)=\left(\begin{array}{cc}
a+i b & \\
& a-i b
\end{array}\right) ;
$$

then $\operatorname{Ad}(x): S(\mathbb{C}) \stackrel{\sim}{\rightarrow} A_{0}(\mathbb{C})$ is identical to $\varphi$, viewing these two tori under the embeddings above. 
We fix the isomorphism from $\mathbb{Z}^{2}$ to $X^{*}(S)$ that sends $(1,0)$ and $(0,1)$ to the character $\varphi$ composed with projection to the first and, respectively, second component of $\mathbb{G}_{m} \times \mathbb{G}_{m}$. Similarly we fix the isomorphism from $\mathbb{Z}^{2}$ to $X_{*}(S)$ that sends $(1,0)$ and $(0,1)$ to the cocharacters $a \mapsto \varphi^{-1}(a, 1)$ and $a \mapsto \varphi^{-1}(1, a)$, respectively.

Write $\hat{S}$ for the Langlands dual torus to $S$. It is isomorphic to $\mathbb{C}^{\times} \times \mathbb{C}^{\times}$as a group, with $\Gamma_{\mathbb{R}}$-action defined by $\sigma(\alpha, \beta)=(\beta, \alpha)$. We fix the isomorphism $X^{*}(S) \stackrel{\sim}{\rightarrow} X_{*}(\hat{S})$ given by $(a, b) \mapsto\left(z \mapsto\left(z^{a}, z^{b}\right)\right)$.

We have an inclusion $\iota_{S}: \mathbb{G}_{m} \rightarrow S$ given on $\mathscr{A}$-points by $a \mapsto a \otimes 1$. Write $\sigma_{S}$ for the automorphism of $S$ given by $1 \otimes \sigma$ on $\mathscr{A}$-points. Note that the fixed point set of $\sigma_{S}$ is precisely the image of $\iota_{S}$.

Write Nm $: S \rightarrow \mathbb{G}_{m}$ for the norm map given by $s \mapsto s \cdot \sigma_{S}(s)$. Note that the product $s \cdot \sigma_{S}(s)$ is in $\iota_{S}\left(\mathbb{G}_{m}\right)$, which we identify here with $\mathbb{G}_{m}$. One computes that the norm map induces the map $n \mapsto(n, n)$ from $X^{*}\left(\mathbb{G}_{m}\right)$ to $X^{*}(S)$ with the identifications above.

Write $T_{1}$ for the kernel of this norm map. Its group of characters fits into the exact sequence

$$
0 \rightarrow X^{*}\left(\mathbb{G}_{m}\right) \rightarrow X^{*}(S) \rightarrow X^{*}\left(T_{1}\right) \rightarrow 0 .
$$

We identify $X^{*}\left(T_{1}\right)$ with $\mathbb{Z}$ so that the restriction map $X^{*}(S) \rightarrow X^{*}\left(T_{1}\right)$ is given by $(a, b) \mapsto a-b$. The corresponding map $\hat{S} \rightarrow \hat{T}$ is given by $(\alpha, \beta) \mapsto \alpha \beta^{-1}$.

\subsection{The kernel and cokernel tori.}

Definition 21. We define $A_{\mathrm{ker}}$ to be the kernel of the map from $\mathbb{G}_{m}{ }^{4} \rightarrow \mathbb{G}_{m}$ given by $(a, b, c, d) \mapsto(a b) /(c d)$. We define $A_{\mathrm{cok}}$ to be the cokernel of the map from $\mathbb{G}_{m}$ to $\mathbb{G}_{m}{ }^{4}$ given by $x \mapsto\left(x, x, x^{-1}, x^{-1}\right)$. Write $T_{\text {ker }}$ for the kernel of the map

$$
S \times S \rightarrow \mathbb{G}_{m}, \quad(\alpha, \beta) \mapsto \mathrm{Nm}(\alpha / \beta),
$$

and $T_{\text {cok }}$ for the cokernel of the map

$$
\mathbb{G}_{m} \rightarrow S \times S, \quad x \mapsto\left(\iota_{S}(x), \iota_{S}\left(x^{-1}\right)\right) .
$$

Identifying $X_{*}\left(\mathbb{G}_{m}\right)$ and $X^{*}\left(\mathbb{G}_{m}\right)$ with $\mathbb{Z}$ as before, we obtain exact sequences

$$
\begin{aligned}
& 0 \rightarrow X_{*}\left(A_{\mathrm{ker}}\right) \rightarrow \mathbb{Z}^{4} \rightarrow \mathbb{Z} \rightarrow 0, \\
& 0 \rightarrow \mathbb{Z} \rightarrow \mathbb{Z}^{4} \rightarrow X^{*}\left(A_{\mathrm{ker}}\right) \rightarrow 0, \\
& 0 \rightarrow \mathbb{Z} \rightarrow \mathbb{Z}^{4} \rightarrow X_{*}\left(A_{\mathrm{cok}}\right) \rightarrow 0, \\
& 0 \rightarrow X^{*}\left(A_{\mathrm{cok}}\right) \rightarrow \mathbb{Z}^{4} \rightarrow \mathbb{Z} \rightarrow 0 .
\end{aligned}
$$

Here the maps from $\mathbb{Z} \rightarrow \mathbb{Z}^{4}$ are both $n \mapsto(n, n,-n,-n)$, and the maps from $\mathbb{Z}^{4} \rightarrow \mathbb{Z}$ are both $\left(n_{1}, n_{2}, n_{3}, n_{4}\right) \mapsto n_{1}+n_{2}-n_{3}-n_{4}$. 
Thus we obtain isomorphisms

$$
g_{\mathrm{kc}}: X^{*}\left(A_{\mathrm{ker}}\right) \stackrel{\sim}{\rightarrow} X_{*}\left(A_{\mathrm{cok}}\right) \quad \text { and } \quad g_{\mathrm{ck}}: X^{*}\left(A_{\mathrm{cok}}\right) \stackrel{\sim}{\rightarrow} X_{*}\left(A_{\mathrm{ker}}\right),
$$

obtained from the exact sequences defining $A_{\mathrm{ker}}$ and $A_{\mathrm{cok}}$. In this way we view $A_{\text {cok }}(\mathbb{C})$ and $A_{\text {ker }}(\mathbb{C})$ as the dual tori $\hat{A}_{\text {ker }}$ and $\hat{A}_{\text {cok }}$, respectively.

The isomorphism $\varphi \times \varphi: S(\mathbb{C}) \times S(\mathbb{C}) \stackrel{\sim}{\rightarrow}\left(\mathbb{C}^{\times}\right)^{4}$ gives isomorphisms $\Phi_{\mathrm{ker}}$ : $T_{\text {ker }}(\mathbb{C}) \stackrel{\sim}{\rightarrow} A_{\text {ker }}(\mathbb{C})$ and $\Phi_{\text {cok }}: T_{\text {cok }}(\mathbb{C}) \stackrel{\sim}{\rightarrow} A_{\text {cok }}(\mathbb{C})$.

Consider the map from $S \times S$ to $S \times S$ given by $(a, b) \mapsto\left(a b, a \sigma_{S}(b)\right)$. This fits together with the previous maps to form an exact sequence

$$
1 \rightarrow \mathbb{G}_{m} \rightarrow S \times S \rightarrow S \times S \rightarrow \mathbb{G}_{m} \rightarrow 1,
$$

and yields an isomorphism $\Psi_{T}: T_{\text {cok }} \stackrel{\sim}{\rightarrow} T_{\text {ker }}$.

Consider the map from $\mathbb{G}_{m}{ }^{4}$ to $\mathbb{G}_{m}{ }^{4}$ given by $(a, b, c, d) \mapsto(a c, b d, a d, b c)$. This fits together with the previous maps to form an exact sequence

$$
1 \rightarrow \mathbb{G}_{m} \rightarrow \mathbb{G}_{m}{ }^{4} \rightarrow \mathbb{G}_{m}{ }^{4} \rightarrow \mathbb{G}_{m} \rightarrow 1
$$

and yields an isomorphism $\Psi_{A}: A_{\text {cok }} \stackrel{\sim}{\rightarrow} A_{\text {ker. }}$ On $\mathbb{C}$-points we have

$$
\Phi_{\mathrm{ker}} \circ \Psi_{T}(\mathbb{C})=\Psi_{A}(\mathbb{C}) \circ \Phi_{\mathrm{cok}}
$$

\section{Structure of $\operatorname{GSp}_{4}(F)$}

9.1. The general symplectic group. Let $F$ be a field of characteristic 0 . Put

$$
J=\left(\begin{array}{llll} 
& & & 1 \\
& & -1 & \\
& 1 & &
\end{array}\right)
$$

Take $G$ to be the algebraic group $\mathrm{GSp}_{4}=\left\{g \in \mathrm{GL}_{4} \mid g J g^{t}=\mu J\right.$, some $\mu=$ $\left.\mu(g) \in \mathbb{G}_{m}\right\}$. It is closely related to the group $G^{\prime}=\operatorname{Sp}_{4}=\left\{g \in \mathrm{GSp}_{4} \mid \mu(g)=1\right\}$. Write $A$ for the subgroup of diagonal matrices in $G$, and $Z$ for the subgroup of scalar matrices in $G$.

We fix the isomorphism $\iota_{A}: A_{\text {ker }} \stackrel{\sim}{\rightarrow} A$ given by

$$
(a, b, c, d) \mapsto \operatorname{diag}(a, c, d, b) .
$$

Let $B_{A}$ be the Borel subgroup of upper triangular matrices in $G$.

9.2. Root data. Although $A$ and $A_{\text {ker }}$ are isomorphic tori, we prefer to parametrize their character and cocharacter groups differently, since the isomorphism $\iota_{A}$ permutes the order of the components. 
So we express $X^{*}(A)=\operatorname{Hom}\left(A, \mathbb{G}_{m}\right)$ as the cokernel of the map

$$
i: \mathbb{Z} \rightarrow \mathbb{Z}^{4}
$$

given by $i(n)=(n,-n,-n, n)$.

We write $e_{1}, \ldots, e_{4}$ for the images in $X^{*}(A)$ of $(1,0,0,0), \ldots,(0,0,0,1)$. Thus $e_{1}+e_{4}=e_{2}+e_{3}$. The basis $\Delta_{G}$ of simple roots corresponding to $B_{A}$ is $\left\{e_{1}-e_{2}, e_{2}-e_{3}\right\}$, with corresponding positive roots $\left\{e_{1}-e_{2}, e_{1}-e_{4}, e_{2}-e_{3}, e_{1}-e_{3}\right\}$. The half-sum of the positive roots is then $\rho_{B}=\frac{1}{2}\left(4 e_{1}-e_{2}-3 e_{3}\right) \in X^{*}(A)$.

Definition 22. Write $\Omega$ for the Weyl group of $A$ in $G$. Write $w_{0}, w_{1}, w_{2}$ for the elements of $\Omega$ that conjugate $\operatorname{diag}(a, b, c, d) \in A$ to

$$
\operatorname{diag}(d, c, b, a), \quad \operatorname{diag}(a, c, b, d), \quad \operatorname{diag}(b, a, d, c),
$$

respectively.

$\Omega$ has order 8 and is generated by $w_{0}, w_{1}$, and $w_{2}$.

Express $X_{*}(A)$ as the kernel of the map

$$
p: \mathbb{Z}^{4} \rightarrow \mathbb{Z}, \quad(a, b, c, d) \mapsto a-b-c+d .
$$

Let $\vartheta_{1}=(1,0,0,-1)$ and $\vartheta_{2}=(0,1,-1,0) \in X_{*}(A)$. Then the coroots of $A$ in $G$ are given by $R^{\vee}=R^{\vee}(A, G)=\left\{ \pm \vartheta_{1} \pm \vartheta_{2}, \pm \vartheta_{1}, \pm \vartheta_{2}\right\}$. The basis $\Delta_{G}^{\vee}$ of simple coroots dual to $\Delta_{G}$ is $\left\{\vartheta_{1}-\vartheta_{2}, \vartheta_{2}\right\}$. Then $\left(X^{*}(A), \Delta_{G}, X_{*}(A), \Delta_{G}^{\vee}\right)$ is a based root datum for $G$.

9.3. The dual group $\hat{\boldsymbol{G}}$. We will take $\hat{G}$ to be $\mathrm{GSp}_{4}(\mathbb{C})$, with trivial $L$-action, and the same based root data as already discussed for $G$. The isomorphism

$$
X^{*}(A) \stackrel{\left(\iota_{A}\right)^{*}}{\longrightarrow} X^{*}\left(A_{\mathrm{ker}}\right) \stackrel{\left(\Psi_{A}\right)^{*}}{\longrightarrow} X^{*}\left(A_{\mathrm{cok}}\right) \stackrel{g_{c k}}{\longrightarrow} X_{*}\left(A_{\mathrm{ker}}\right) \stackrel{\left(\iota_{A}\right)_{*}}{\longrightarrow} X_{*}(A)
$$

(and its inverse) furnish the required isomorphism of based root data. Let us write this out more explicitly. Note that $\left(\iota_{A}\right)_{*}$ and $\left(\iota_{A}\right)^{*}$ are given by

$$
\left(\iota_{A}\right)_{*}(a, b, c, d)=(a, c, d, b) \quad \text { and } \quad\left(\iota_{A}\right)^{*}(a, b, c, d)=(a, d, b, c) .
$$

The isomorphism in (9-4) is induced from the linear transformation $\Sigma: \mathbb{Z}^{4} \rightarrow \mathbb{Z}^{4}$ represented by the matrix

$$
\left(\begin{array}{llll}
1 & 1 & 0 & 0 \\
1 & 0 & 1 & 0 \\
0 & 1 & 0 & 1 \\
0 & 0 & 1 & 1
\end{array}\right),
$$


which gives the exact sequence $0 \rightarrow \mathbb{Z} \stackrel{i}{\rightarrow} \mathbb{Z}^{4} \stackrel{\Sigma}{\rightarrow} \mathbb{Z}^{4} \stackrel{p}{\rightarrow} \mathbb{Z} \rightarrow 0$, and thus an isomorphism

$$
X^{*}(A) \stackrel{\Sigma}{\stackrel{\Sigma}{\longrightarrow}} X_{*}(A)
$$

This agrees with the isomorphism used in [Roberts and Schmidt 2007, Section 2.3].

We have $\Sigma\left(e_{1}-e_{2}\right)=\vartheta_{2}$ and $\Sigma\left(e_{2}-e_{3}\right)=\vartheta_{1}-\vartheta_{2}$. Thus the based root datum above is self-dual. Note that $\Sigma(\rho)=\frac{3}{2} \vartheta_{1}+\frac{1}{2} \vartheta_{2}$. Write $\hat{A}$ for $A(\mathbb{C})$; it is the torus dual to $A$ via the isomorphism in (9-5).

\section{Discrete series for $\mathrm{GSp}_{4}(\mathbb{R})$}

10.1. The maximal elliptic torus $\boldsymbol{T}$ of $\boldsymbol{G}$. Consider the map $\mathrm{GL}_{2} \times \mathrm{GL}_{2} \rightarrow \mathrm{GL}_{4}$ given by

$$
\left(\begin{array}{ll}
a & b \\
c & d
\end{array}\right) \times\left(\begin{array}{ll}
e & f \\
g & h
\end{array}\right) \mapsto\left(\begin{array}{llll}
a & & & b \\
& e & f & \\
& g & h & \\
c & & & d
\end{array}\right)
$$

The composition of this with the natural inclusion $S \times S \rightarrow \mathrm{GL}_{2} \times \mathrm{GL}_{2}$ gives an embedding of $S \times S$ into $\mathrm{GL}_{4}$. This restricts to an embedding of $T_{\text {ker }}$ into $G$, whose image is an elliptic maximal torus $T$ of $G$. Thus we have $\iota_{T}: T_{\text {ker }} \stackrel{\sim}{\rightarrow} T$.

$T(\mathbb{R})$ is the subgroup of matrices of the form

$$
\gamma_{r, \theta_{1}, \theta_{2}}=\left(\begin{array}{cccc}
r \cos \left(\theta_{1}\right) & & & -r \sin \left(\theta_{1}\right) \\
& r \cos \left(\theta_{2}\right) & -r \sin \left(\theta_{2}\right) & \\
& r \sin \left(\theta_{2}\right) & r \cos \left(\theta_{2}\right) & \\
r \sin \left(\theta_{1}\right) & & & r \cos \left(\theta_{1}\right)
\end{array}\right)
$$

for $r>0$ and angles $\theta_{1}, \theta_{2}$.

Pick an element $\xi \in G(\mathbb{C})$ so that

$$
\operatorname{Ad}(\xi)\left(\begin{array}{llll}
a & & & -b \\
& c & -d & \\
& d & c & \\
b & & a
\end{array}\right)=\left(\begin{array}{llll}
a+i b & & & \\
& c+i d & & \\
& & c-i d & \\
& & & a-i b
\end{array}\right),
$$

and put $B_{T}=\operatorname{Ad}\left(\xi^{-1}\right) B_{A}$. Then $B_{T}$ is a Borel subgroup of $G_{\mathbb{C}}$ containing $T$, and $\operatorname{Ad}(\xi): T(\mathbb{C}) \stackrel{\sim}{\rightarrow} A(\mathbb{C})$ is the canonical isomorphism associated to the pairs $\left(T, B_{T}\right)$ and $\left(A, B_{A}\right)$. The definitions have been set up so that

$$
\iota_{A} \circ \Phi_{\mathrm{ker}}=\operatorname{Ad}(\xi) \circ \iota_{T}
$$


We identify $A(\mathbb{C})$ as the torus dual $\hat{T}$ to $T$ via the isomorphisms

$$
\begin{aligned}
X^{*}(T) \stackrel{\left(\iota_{T}\right)^{*}}{\longrightarrow} X^{*}\left(T_{\mathrm{ker}}\right) \stackrel{\Phi_{\mathrm{ker}}^{*}}{\longrightarrow} & X^{*}\left(A_{\mathrm{ker}}\right) \\
& \stackrel{\left(\Psi_{A}\right)^{*}}{\longrightarrow} X^{*}\left(A_{\mathrm{cok}}\right) \stackrel{g_{c k}}{\longrightarrow} X_{*}\left(A_{\mathrm{ker}}\right) \stackrel{\left(\iota_{A}\right)_{*}}{\longrightarrow} X_{*}(A) .
\end{aligned}
$$

10.2. Real Weyl group. We use $\operatorname{Ad}(\xi)$ to identify $\Omega$ with the Weyl group of $T(\mathbb{C})$ in $G(\mathbb{C})$. Recall that $\Omega_{\mathbb{R}}$ denotes the Weyl group of $T(\mathbb{R})$ in $G(\mathbb{R})$. By [Warner 1972, Proposition 1.4.2.1], we have

$$
\Omega_{\mathbb{R}}=N_{K_{\mathbb{R}}}(T(\mathbb{R})) /\left(T(\mathbb{R}) \cap K_{\mathbb{R}}\right) .
$$

When discussing maximal compact subgroups of $\mathrm{GSp}_{4}(\mathbb{R})$, it is convenient to use a different realization of these symplectic groups. Following [Pitale and Schmidt 2009], take for $J$ the symplectic matrix

$$
\left(\begin{array}{llll} 
& & 1 & \\
& & & 1 \\
-1 & & & \\
& -1 & &
\end{array}\right) \text {. }
$$

Take for $K_{\mathbb{R}}$ the standard maximal compact subgroup of $\mathrm{GSp}_{4}(\mathbb{R})$ (the intersection of $G(\mathbb{R})$ with the orthogonal group), and $S K_{\mathbb{R}}$ the intersection of $K_{\mathbb{R}}$ with $\mathrm{Sp}_{4}(\mathbb{R})$. One finds that $S K_{\mathbb{R}}$ is isomorphic to the compact unitary group $U_{2}(\mathbb{R})$, and yields the Weyl group element $w_{2}$. The element $\operatorname{diag}(1,1,-1,-1) \in N_{G(\mathbb{R})}(T(\mathbb{R})) \cap K_{\mathbb{R}}$ gives $w_{0} \in \Omega_{\mathbb{R}}$, and these two elements generate $\Omega_{\mathbb{R}}$. This subgroup has index 2 in $\Omega$, and does not contain the element $w_{1}$.

10.3. Admissible embeddings. Consider the admissible embedding $\eta_{B}:{ }^{L} T \rightarrow{ }^{L} G$. Write $\theta(z)=z /|z|$ for $z \in \mathbb{C}^{\times}$. We have ${ }^{L} T=\hat{T} \rtimes W_{\mathbb{R}}$, with $\tau$ acting as the longest Weyl group element on $\hat{T}$.

Writing ${ }^{L} T=\hat{T} \times W_{\mathbb{R}}$, we put

$$
\begin{aligned}
& \eta_{B}(1 \times z)=\operatorname{diag}\left(\theta(z)^{3}, \theta(z), \theta(z)^{-1}, \theta(z)^{-3}\right) \times z \quad \text { for } z \in \mathbb{C}^{\times} \cong W_{\mathbb{C}}, \\
& \eta_{B}(\hat{t} \times 1)=\hat{t} \times 1 \quad \text { for } \hat{t} \in \hat{T} \text {, } \\
& \eta_{B}(1 \times \tau)=J \times \tau .
\end{aligned}
$$

10.4. Elliptic Langlands parameters. Let $a, b$ be odd integers with $a>b>0$. Let $t$ be an even integer. Put

$$
\mu=\frac{1}{2}[(t, t, t, t)+(a, b,-b,-a)] \quad \text { and } \quad v=\frac{1}{2}[(t, t, t, t)+(-a,-b, b, a)],
$$

viewed in $X_{*}(\hat{T})_{\mathbb{C}}$. Then we may define a Langlands parameter $\varphi_{G}: W_{\mathbb{R}} \rightarrow{ }^{L} G$ by

$$
\varphi_{G}(z)=z^{\mu} \bar{z}^{v} \times z=|z|^{t} \operatorname{diag}\left(\theta(z)^{a}, \theta(z)^{b}, \theta(z)^{-b}, \theta(z)^{-a}\right) \times z,
$$


and $\varphi_{G}(\tau)=J \times \tau$.

Note that the centralizer of $\varphi_{G}\left(W_{\mathbb{C}}\right)$ in $\hat{G}$ is simply $\hat{A}$, and that $\langle\mu, \alpha\rangle$ is positive for every root of $A$ that is positive for $B_{A}(\mathbb{C})$. Thus $\varphi_{G}$ determines the pair $\left(\hat{A}, \hat{B}_{A}\right)$, where $\hat{B}_{A}$ is simply $B_{A}(\mathbb{C})$.

Define a Langlands parameter $\varphi_{B}: W_{\mathbb{R}} \rightarrow{ }^{L} T$ by

$$
\varphi_{B}(z)=|z|^{t} \operatorname{diag}\left(\theta(z)^{a-3}, \theta(z)^{b-1}, \theta(z)^{1-b}, \theta(z)^{3-a}\right) \times z,
$$

and $\varphi_{B}(\tau)=1 \times \tau$. Then $\varphi_{G}=\eta_{B} \circ \varphi_{B}$.

Let $\pi_{G}=\pi\left(\varphi_{G}, B_{T}\right)$ and $\pi_{G}^{\prime}=\pi\left(\varphi_{G}, w_{1}\left(B_{T}\right)\right)$, with notation from Section 2.2. The $L$-packet determined by $\varphi_{G}$ is $\Pi=\left\{\pi_{G}, \pi_{G}^{\prime}\right\}$. Here $\pi_{G}$ is called a holomorphic discrete series representation, and $\pi_{G}^{\prime}$ is called a large discrete series representation.

The highest weight for the associated representation $E$ of $G(\mathbb{C})$ is

$$
\lambda_{B}=\frac{1}{2}(a+b-4, t-b+1, t-a+3,0) \in X^{*}(A) .
$$

From this we may read off the central character $\lambda_{E}(z I)=z^{t}$ for $z I \in A_{G}(\mathbb{C})$.

\section{The elliptic endoscopic group $H$}

11.1. Root data. Let $H$ be the cokernel of the map $\mathbb{G}_{m} \rightarrow \mathrm{GL}_{2} \times \mathrm{GL}_{2}$ given by $t \mapsto t I \times t^{-1} I$. Write $A^{H}$ for the diagonal matrices in $H$, and $B_{H}$ for the pairs of upper triangular matrices in $H$. Fix $\iota_{A^{H}}: A_{\text {cok }} \stackrel{\sim}{\rightarrow} A^{H}$ given by

$$
(a, b, c, d) \mapsto \operatorname{diag}(a, b) \times \operatorname{diag}(d, c) .
$$

Write $T_{H}$ for the image of $S \times S$ in $H$. It is an elliptic maximal torus in $H$. Fix $\iota_{T_{H}}: T_{\mathrm{cok}} \stackrel{\sim}{\rightarrow} T_{H}$ obtained from the map $S \times S \rightarrow \mathrm{GL}_{2} \times \mathrm{GL}_{2}, \alpha \mapsto\left(\iota_{S}(\alpha), \iota_{S}(\alpha)\right)$. Put $B_{T_{H}}=\operatorname{Ad}(x \times x)^{-1} B_{H}$, a Borel subgroup of $H_{\mathbb{C}}$ containing $T_{H}$. Then $\operatorname{Ad}(x \times x)$ is the canonical isomorphism $T_{H}(\mathbb{C}) \stackrel{\sim}{\rightarrow} A^{H}(\mathbb{C})$ associated to the pairs $\left(T_{H}, B_{T_{H}}\right)$ and $\left(A^{H}, B_{H}\right)$. We view $X^{*}\left(T_{H}\right)$ as the kernel of the map $p: \mathbb{Z}^{2} \times \mathbb{Z}^{2} \rightarrow \mathbb{Z}$ given by $(a, b) \times(c, d) \mapsto a+b-c-d$. We have a basis of roots $\Delta_{H}$ given by

$$
\Delta_{H}=\{(1,-1) \times(0,0),(0,0) \times(1,-1)\},
$$

and $\rho_{H}=\frac{1}{2}(1,-1) \times \frac{1}{2}(1,-1)$.

Furthermore, $X_{*}\left(T_{H}\right)$ is the cokernel of the map $\iota: \mathbb{Z} \rightarrow \mathbb{Z}^{2} \times \mathbb{Z}^{2}$ given by $a \mapsto(a, a) \times(-a,-a)$. We have a basis of coroots $\Delta_{H}^{\vee}$ given by

$$
\Delta_{H}^{\vee}=\{(1,-1) \times(0,0),(0,0) \times(1,-1)\},
$$

viewed in the quotient $X_{*}\left(T_{H}\right)$. 
11.2. Dual group $\hat{\boldsymbol{H}}$. Let $\hat{H}=\left\{(g, h) \in \mathrm{GL}_{2}(\mathbb{C}) \times \mathrm{GL}_{2}(\mathbb{C}) \mid \operatorname{det}(g)=\operatorname{det}(h)\right\}$. We have an inclusion $A_{\text {ker }}(\mathbb{C}) \rightarrow \hat{H}$ given by

$$
(a, b, c, d) \mapsto \operatorname{diag}(a, b) \times \operatorname{diag}(d, c) .
$$

Write $\hat{A}^{H} \subset \hat{H}$ for the image. We thus have an isomorphism $\iota_{\hat{A}^{H}}: A_{\mathrm{ker}}(\mathbb{C}) \stackrel{\sim}{\rightarrow} \hat{A}^{H}$. Also write $\hat{B}_{H}$ for the subgroup of upper triangular matrices in $\hat{H}$. This Borel subgroup determines a based root datum for $\hat{H}$.

Giving $\hat{H}$ the trivial $L$-action, we view it as a dual group to $H$ via the isomorphisms

$$
\begin{aligned}
& X^{*}\left(A^{H}\right) \stackrel{\left(\iota_{A}\right)^{*}}{\longrightarrow} X^{*}\left(A_{\mathrm{cok}}\right) \stackrel{g_{\mathrm{ck}}}{\longrightarrow} X_{*}\left(A_{\mathrm{ker}}\right) \stackrel{\left({ }_{\hat{A}^{H}}\right)_{*}}{\longrightarrow} X_{*}\left(\hat{A}^{H}\right), \\
& X^{*}\left(\hat{A}^{H}\right) \stackrel{\left(\iota_{\hat{A}}\right)^{*}}{\longrightarrow} X^{*}\left(A_{\mathrm{ker}}\right) \stackrel{g_{\mathrm{kc}}}{\longrightarrow} X_{*}\left(A_{\mathrm{cok}}\right) \stackrel{\left({ }_{\iota_{A}}\right)_{*}}{\longrightarrow} X_{*}\left(A^{H}\right) .
\end{aligned}
$$

We identify $\hat{A}^{H}$ as the torus $\hat{T}_{H}$ dual to $T_{H}$ via the isomorphisms

$$
X^{*}\left(T_{H}\right) \stackrel{\left(\iota_{T_{H}}\right)^{*}}{\longrightarrow} X^{*}\left(T_{\mathrm{cok}}\right) \stackrel{\Phi_{\mathrm{cok}}^{*}}{\longrightarrow} X^{*}\left(A_{\mathrm{cok}}\right) \stackrel{g_{c k}}{\longrightarrow} X_{*}\left(A_{\mathrm{ker}}\right) \stackrel{\left({ }_{\hat{A}^{H}}\right)^{*}}{\longrightarrow} X_{*}\left(\hat{A}^{H}\right) .
$$

Let $\eta:{ }^{L} H \rightarrow{ }^{L} G$ be given by

$$
\left(\begin{array}{ll}
a & b \\
c & d
\end{array}\right) \times\left(\begin{array}{ll}
e & f \\
g & h
\end{array}\right) \times w \mapsto\left(\begin{array}{llll}
a & & & b \\
& e & f & \\
& g & h & \\
c & & & d
\end{array}\right) \times w .
$$

Let $s=\operatorname{diag}(1,1) \times \operatorname{diag}(-1,-1) \in \hat{H}$.

The image $\eta(\hat{H})$ is the connected centralizer in $\hat{G}$ of $\eta(s)$. Thus, $(H, s, \eta)$ is an elliptic endoscopic triple for $G$. In fact it is the only one, up to isomorphism.

Moreover note that $\eta$ restricted to $\hat{A}^{H}$ is given by

$$
\left.\eta\right|_{\hat{A}^{H}}=\iota_{A} \circ\left(\iota_{\hat{A}^{H}}\right)^{-1} \text {. }
$$

(Recall that $\hat{A}=A(\mathbb{C})$.)

\section{Transfer for $H(\mathbb{R})$}

The goal of this section is Proposition 12, in which we identify $e_{\pi_{G}}^{H}$ and $e_{\pi_{G}^{\prime}}^{H}$. This is part of the global transfer $f^{H} d h$ that is to be entered into $S T_{g}$ for the endoscopic group $H$. We will recognize it using the character theory of transfer reviewed in Section 3.

12.1. Parametrization of discrete series. First we must set up the Langlands parameters for discrete series representations of $H(\mathbb{R})$, and describe how they transfer to $L$-packets in $G(\mathbb{R})$. Recall that we have fixed three integers $a, b, t$, with $a, b$ odd, 
$t$ even, and $a>b>0$. Define the Langlands parameter $\varphi_{H}: W_{\mathbb{R}} \rightarrow{ }^{L} H=\hat{H} \times W_{\mathbb{R}}$ by

$$
\varphi_{H}(z)=|z|^{t} \operatorname{diag}\left(\theta(z)^{a}, \theta(z)^{-a}\right) \times|z|^{t} \operatorname{diag}\left(\theta(z)^{b}, \theta(z)^{-b}\right) \times z
$$

for $z \in W_{\mathbb{C}}$, and

$$
\varphi_{H}(\tau)=\left({ }_{-1}{ }^{1}\right) \times\left({ }_{1}{ }^{-1}\right) \times \tau .
$$

Then $\varphi_{H}$ determines the pair $\left(\hat{A}_{H}, \hat{B}_{H}\right)$. The $L$-packet is a singleton $\left\{\pi_{H}\right\}$. The corresponding representation $E_{H}$ of $H(\mathbb{C})$ has highest weight

$$
\lambda_{H}=\frac{1}{2}(t+a-1, t-a+1) \times \frac{1}{2}(t+b-1, t-b+1)
$$

and central character $\lambda_{E_{H}}\left(z_{1}, z_{2}\right)=\left(z_{1} z_{2}\right)^{t}$. Most importantly, we have $\varphi_{G}=\eta \circ \varphi_{H}$.

There is another Langlands parameter $\varphi_{H}^{\prime}$ given by

$$
\varphi_{H}^{\prime}(z)=|z|^{t} \operatorname{diag}\left(\theta(z)^{b}, \theta(z)^{-b}\right) \times|z|^{t} \operatorname{diag}\left(\theta(z)^{a}, \theta(z)^{-a}\right) \times z,
$$

and by $\varphi_{H}^{\prime}(\tau)=\varphi_{H}(\tau)$ as above.

Again the $L$-packet is a singleton $\left\{\pi_{H}^{\prime}\right\}$. The corresponding representation $E_{H}^{\prime}$ has highest weight

$$
\lambda_{H}^{\prime}=\frac{1}{2}(t+b-1, t-b+1) \times \frac{1}{2}(t+a-1, t-a+1),
$$

and central character $\lambda_{E_{H}^{\prime}}=\lambda_{E_{H}}$ above.

Let $\varphi_{G}^{\prime}=\eta \circ \varphi_{H}^{\prime}$. Then $\varphi_{G}^{\prime}=\operatorname{Int}\left(w_{2}\right) \circ \varphi_{G}$, so it is equivalent to $\varphi_{G}$. In particular, both $L$-packets $\left\{\pi_{H}\right\}$ and $\left\{\pi_{H}^{\prime}\right\}$ transfer to $\Pi=\left\{\pi_{G}, \pi_{G}^{\prime}\right\}$.

12.2. Alignment. Recall the definition of alignment from Section 3.

Lemma 8. Define $j: T_{H} \stackrel{\sim}{\rightarrow} T$ by $j=\iota_{T} \circ \Psi_{T} \circ\left(\iota_{T_{H}}\right)^{-1}$. Then $\left(j, B_{T}, B_{T_{H}}\right)$ is aligned with $\varphi_{H}$, and $\left(j, w_{1} B_{T}, B_{T_{H}}\right)$ is aligned with $\varphi_{H}^{\prime}$.

Proof. Since the parameter $\varphi_{G}$ gives the pair $(\hat{A}, \hat{B})$, the parameter $\varphi_{G}^{\prime}$ gives the pair $\left(\hat{A}, w_{1} \hat{B}\right)$, and because $\varphi_{H}$ and $\varphi_{H}^{\prime}$ both give $\left(\hat{A}, \hat{B}_{H}\right)$, the horizontal maps in (3-2) are identities. The map $\hat{j}: \hat{T} \rightarrow \hat{T}_{H}$ may be computed by composing the isomorphism $X_{*}(\hat{T}) \stackrel{\sim}{\rightarrow} X^{*}(T)$ in $(10-2)$ with the induced map $j^{*}: X^{*}(T) \stackrel{\sim}{\rightarrow} X^{*}\left(T_{H}\right)$ and finally with the inverse of the isomorphism $X_{*}\left(\hat{T}_{H}\right) \stackrel{\sim}{\rightarrow} X^{*}\left(T_{H}\right)$ in (11-3). Using equations (8-1) and (11-5), one finds that $\hat{j}=\iota_{\hat{A}_{H}} \circ\left(\iota_{A}\right)^{-1}=\eta^{-1}$, as desired.

\subsection{Transfer for $\boldsymbol{H}_{\mathbb{R}}$.}

Proposition 12. Let $\pi_{G}=\pi\left(\varphi_{G}, B_{T}\right)$ and $\pi_{G}^{\prime}=\pi\left(\varphi_{G}, \omega^{-1}\left(B_{T}\right)\right)$ as described in Section 10.4. Then (using notation from Section 2.4) we may take $e_{\pi_{G}}^{H}=e_{\pi_{H}}+e_{\pi_{H}^{\prime}}$, where $\pi_{H}$ and $\pi_{H}^{\prime}$ are the discrete series representation determined by $\varphi_{H}$ and $\varphi_{H}^{\prime}$, respectively, as above. Furthermore, we may take $e_{\pi_{G}^{\prime}}^{H}=-e_{\pi_{G}}^{H}$. 
Proof. By Lemma 8, we may use

$$
\begin{aligned}
\Delta_{\infty}\left(\varphi_{H}, \pi\left(\varphi_{G}, \omega^{-1}\left(B_{T}\right)\right)\right) & =\left\langle a_{\omega}, \hat{j}^{-1}(s)\right\rangle, \\
\Delta_{\infty}\left(\varphi_{H}^{\prime}, \pi\left(\varphi_{G}, \omega^{-1}\left(w_{1} B_{T}\right)\right)\right) & =\left\langle a_{w_{1} \omega}, \hat{j}^{-1}(s)\right\rangle
\end{aligned}
$$

for $\omega \in \Omega$. In both cases, this is given by

$$
\left\langle a_{\omega}, s\right\rangle=\left\{\begin{aligned}
1 & \text { if } \omega \in \Omega_{\mathbb{R}}, \\
-1 & \text { if } \omega \notin \Omega_{\mathbb{R}} .
\end{aligned}\right.
$$

Note that $\left\langle a_{w_{1} \omega}, \hat{j}^{-1}(s)\right\rangle=-\left\langle a_{\omega}, \hat{j}^{-1}(s)\right\rangle$. Therefore the characterization (3-1) becomes, for a general measure $f_{\infty} d g_{\infty}$ at the real place,

$$
\begin{aligned}
\Theta_{\pi_{H}}\left(f_{\infty}^{H} d h_{\infty}\right) & =\sum_{\pi \in \Pi\left(\varphi_{G}\right)} \Delta_{\infty}\left(\varphi_{H}, \pi\right) \Theta_{\pi}\left(f_{\infty} d g_{\infty}\right) \\
& =\Theta_{\pi_{G}}\left(f_{\infty} d g_{\infty}\right)-\Theta_{\pi_{G}^{\prime}}\left(f_{\infty} d g_{\infty}\right)
\end{aligned}
$$

and similarly

$$
\Theta_{\pi_{H}^{\prime}}\left(f_{\infty}^{H} d h_{\infty}\right)=\Theta_{\pi_{G}}\left(f_{\infty} d g_{\infty}\right)-\Theta_{\pi_{G}^{\prime}}\left(f_{\infty} d g_{\infty}\right) .
$$

In our case, we obtain

$$
\Theta_{\pi_{H}}\left(e_{\pi_{G}}^{H}\right)=\Theta_{\pi_{H}^{\prime}}\left(e_{\pi_{G}}^{H}\right)=(-1)^{q(G)} \quad \text { and } \quad \Theta_{\pi_{H}}\left(e_{\pi_{G}^{\prime}}^{H}\right)=\Theta_{\pi_{H}^{\prime}}\left(e_{\pi_{G}^{\prime}}^{H}\right)=-(-1)^{q(G)} \text {. }
$$

The proposition follows.

\section{Levi subgroups}

13.1. Levi subgroups. We give the standard Levi subgroups of $G$, which are those of the parabolic subgroups containing $B_{A}$. We have the group $A$, the group $G$ itself, and the following two Levi subgroups:

$$
\begin{aligned}
& M_{1}=\left\{\operatorname{diag}(g, \lambda g) \mid g \in \mathrm{GL}_{2}, \lambda \in \mathbb{G}_{m}\right\}, \\
& M_{2}=\left\{\operatorname{diag}(a, g, b) \mid g \in \mathrm{GL}_{2}, a, b \in \mathbb{G}_{m}, \operatorname{det}(g)=a b\right\} .
\end{aligned}
$$

Note that both $M_{1}$ and $M_{2}$ are isomorphic to $\mathbb{G}_{m} \times \mathrm{GL}_{2}$.

The group $H$ also has four Levi subgroups, namely $A^{H}$, the group $H$ itself, the image $M_{1}^{H}$ of $\mathrm{GL}_{2} \times A_{0}$ in $H$, and the image $M_{2}^{H}$ of $A_{0} \times \mathrm{GL}_{2}$ in $H$. Note that both $M_{1}^{H}$ and $M_{2}^{H}$ are isomorphic to $\mathrm{GL}_{2} \times \mathbb{G}_{m}$.

13.2. Miscellaneous constants. We now compute the invariants from Section 5.1 for the Levi subgroups of $G$ and $H$.

First, we compute the various $k(M)$. When $M$ is the split torus $A$ its derived group is trivial and so $k(A)=1$. For $i=1,2$, the Levi subgroup $M_{i}$ is isomorphic 
to $\mathrm{GL}_{2} \times \mathbb{G}_{m}$, and the torus is isomorphic to $S \times \mathbb{G}_{m}$. Since $S$ and $\mathbb{G}_{m}$ have trivial first cohomology, again $k\left(M_{1}\right)=1$.

Lemma 9. We have $k(G)=2$.

Write $T$ as before for the elliptic torus of $G$.

Proof. Recall that $T_{1}$ is the kernel of $\mathrm{Nm}$ and $H^{1}\left(\mathbb{R}, T_{1}\right)$ has order 2.

Recall that the torus $T$ is isomorphic to the kernel of the map

$$
S \times S \rightarrow \mathbb{G}_{m}, \quad(\alpha, \beta) \mapsto \mathrm{Nm}(\alpha / \beta) .
$$

Projection to the first (or second) component followed by Nm gives an exact sequence

$$
1 \rightarrow T_{1} \times T_{1} \rightarrow T \rightarrow \mathbb{G}_{m} \rightarrow 1 .
$$

We have that $G_{\mathrm{sc}}=G_{\mathrm{der}}$ and the inclusion $T_{\mathrm{sc}}=G_{\mathrm{der}} \cap T \subset T$ may be identified with the map $T_{1} \times T_{1} \rightarrow T$ in the sequence above. In particular, $H^{1}\left(\mathbb{R}, T_{\mathrm{sc}}\right)$ has order 4.

Taking the cohomology of (13-1) gives the exact sequence

$$
1 \rightarrow \mathbb{R}^{\times} / \mathbb{R}^{\times 2} \rightarrow H^{1}\left(\mathbb{R}, T_{\mathrm{sc}}\right) \rightarrow H^{1}(\mathbb{R}, T) \rightarrow 1,
$$

from which we conclude that $H^{1}\left(\mathbb{R}, T_{\mathrm{sc}}\right) \rightarrow H^{1}(\mathbb{R}, T)$ is surjective and $H^{1}(\mathbb{R}, T)$ has order 2.

One must also compute $k\left(M_{H}\right)$ for Levi subgroups $M_{H}$ of $H$. The intermediate Levi subgroups are again isomorphic to GL(2) $\times \mathbb{G}_{m}$, and for $A_{H}$ the derived group is trivial. So $k\left(M_{H}\right)=1$ for each of these.

Lemma 10. We have $k(H)=1$.

Proof. We have $T=P(S \times S), \quad H_{\mathrm{sc}}=\mathrm{SL}_{2} \times \mathrm{SL}_{2}$, and $T_{\mathrm{sc}}=T_{1} \times T_{1}$. The map $T_{\mathrm{sc}} \rightarrow T$ factors through $T_{1} \times T_{1} \rightarrow S \times S$. As above we conclude that $k(H)=1$.

Secondly, we compute the Tamagawa numbers. Recall that

$$
\tau(G)=\left|\pi_{0}\left(Z(\hat{G})^{\Gamma_{\mathbb{Q}}}\right)\right| \cdot\left|\operatorname{ker}^{1}(\mathbb{Q}, Z(\hat{G}))\right|^{-1} .
$$

Proposition 13. We have $\tau(M)=1$ for all Levi subgroups of $G$ and for all proper Levi subgroups of $H$, and $\tau(H)=2$.

Proof. For each of these groups, $Z(\hat{M})$ is either the group $\mathbb{C}^{\times}$with trivial $\Gamma_{\mathbb{Q}^{-}}$ action, or a product of such groups. By the Chebotarev density theorem, the homomorphism

$$
\operatorname{Hom}\left(\Gamma_{\mathbb{Q}}, \mathbb{C}^{\times}\right) \rightarrow \prod_{v} \operatorname{Hom}\left(\Gamma_{\mathbb{Q}_{v}}, \mathbb{C}^{\times}\right)
$$


is injective. So $\left|\operatorname{ker}^{1}(\mathbb{Q}, Z(\hat{G}))\right|$ is trivial for our examples. Computing the component group of each $Z(\hat{M})$ is straightforward.

The quantities $n_{M}^{G}$ are easy to compute using $N_{G}(M) \subseteq N_{G}(Z(M))$. If $M$ is a maximal torus, $n_{M}^{G}$ is of course the order of the Weyl group. For the intermediate cases, one finds that $n_{M_{i}}^{G}=n_{M_{i}^{H}}^{H}=2$.

If $\gamma=1$, then $\bar{\imath}^{M}(\gamma)=1$ for each $M$, since each $M$ is connected. Note that for Levi subgroups $M$ of $G$, all proper Levi subgroups $M$ of $H$, and all semisimple elements $\gamma$ in $G$ or $H$, we have $\bar{\iota}^{M}(\gamma)=1$ since in all these cases the derived groups are simply connected.

Finally, we compute $\iota(G, H)$, which we recall is given by

$$
\iota(G, H)=\tau(G) \tau(H)^{-1}|\operatorname{Out}(H, s, \eta)|^{-1} .
$$

One may compute the order of $\operatorname{Out}(H, s, \eta)$ through [Kottwitz 1984, Section 7.6], which shows that this set is in bijection with $\bigwedge(\eta(s), \rho)$, in the notation of that paper. This last set is represented by $\{1, g\}$, where

$$
g=\left(\begin{array}{lll} 
& 1 & \\
1 & & \\
& & \\
& & 1
\end{array}\right) .
$$

The conclusion is that $\iota(G, H)=\frac{1}{4}$.

\section{Computing $S \Phi_{M}$ for Levi subgroups of $G$}

Recall from Proposition 3 the formula

$$
\Phi_{M}\left(\gamma, \Theta^{E}\right)=(-1)^{q(L)}\left|\Omega_{L}\right| \sum_{\omega \in \Omega^{L M}} \varepsilon(\omega) \operatorname{tr}\left(\gamma ; V_{\omega\left(\lambda_{B}+\rho_{B}\right)-\rho_{B}}^{M}\right) \quad \text { for } \gamma \in T_{e}(\mathbb{R}) .
$$

In this section, the maximal torus will be conjugate to $A$, and the character group will be identified with $X^{*}(A)$. We specify an inner product we use on $X^{*}(A)_{\mathbb{R}}$ for the Weyl dimension formula (Proposition 4).

Definition 23. The usual dot product gives an inner product $(\cdot, \cdot)$ on $X_{*}(A)_{\mathbb{R}}$, viewing it as a hypersurface in $\mathbb{R}^{4}$.

Consider the isomorphism

$$
\operatorname{pr}: X^{*}(A)_{\mathbb{R}} \stackrel{\sim}{\rightarrow} X_{*}(A)_{\mathbb{R}}
$$

given by

$$
\operatorname{pr}(a, b, c, d)=(a, b, c, d)-\frac{1}{4}(a+d-b-c)(1,-1,-1,1),
$$

and let $\langle\lambda, \mu\rangle=(\operatorname{pr}(\lambda), \operatorname{pr}(\mu))$. 
For instance,

$$
\operatorname{pr}\left(\lambda_{B}\right)=\frac{1}{4}(a+b+t-4, a-b+t-2,-a+b+t+2,-a-b+t+4) .
$$

It will also be necessary to compute $\Omega^{L M}$ for each example. Recall that this is the set of $w \in \Omega$ such that $w^{-1} \alpha>0$ for positive roots $\alpha$ that are either real or imaginary.

14.1. The term $\Phi_{G}$. By (4-1) we have $\Phi_{G}\left(\gamma, \Theta^{E}\right)=\operatorname{tr}(\gamma ; E)$. Using the Weyl dimension formula, we compute

$$
S \Phi_{G}\left(1, e_{\pi_{G}}\right)=-\frac{1}{24} a b(a+b)(a-b) \bar{v}(G)^{-1} .
$$

14.2. The term $S \Phi_{M_{1}}$. Consider the torus $T_{M_{1}}$ given by

$$
\left(\begin{array}{cccc}
a & b & & \\
-b & a & & \\
& & \lambda a & \lambda b \\
& & -\lambda b & \lambda a
\end{array}\right)
$$

with $a^{2}+b^{2} \neq 0$ and $\lambda \neq 0$. This is an elliptic torus in $M_{1}$.

There is one positive real root $e_{1}-e_{3}$ and one positive imaginary root $\alpha_{M_{1}}=$ $e_{1}-e_{2}$. We have $\Omega^{L M}=\left\{1, w_{1}\right\}, q(L)=1$, and $\left|\Omega_{L}\right|=2$. This gives

$$
\Phi_{M_{1}}\left(1, \Theta^{E}\right)=(-2)\left(\operatorname{dim}_{\mathbb{C}} V_{\lambda_{B}}^{M_{1}}-\operatorname{dim}_{\mathbb{C}} V_{\lambda_{B}^{\prime}}^{M_{1}}\right),
$$

where $\lambda_{B}^{\prime}=\frac{1}{2}(a+b-4, t-a+1, t-b+3,0) \in X^{*}(T)$.

Note that $\left\langle\alpha_{M_{1}}, \lambda_{B}\right\rangle=\frac{1}{2}(b-1)$. The Weyl dimension formula yields

$$
\operatorname{dim}_{\mathbb{C}} V_{\lambda_{B}}^{M_{1}}=b \quad \text { and } \quad \operatorname{dim}_{\mathbb{C}} V_{\lambda_{B}^{\prime}}^{M_{1}}=a .
$$

Thus

$$
S \Phi_{M_{1}}\left(1, e_{\pi_{G}}\right)=-(b-a) \bar{v}\left(M_{1}\right)^{-1} .
$$

14.3. The term $S \Phi_{M_{2}}$. Consider the torus $T_{M_{2}}$ given by

$$
\left(\begin{array}{cccc}
s & & \\
& a & -b & \\
& b & a & \\
& & & t
\end{array}\right),
$$

with $s t=a^{2}+b^{2} \neq 0$. This is an elliptic torus in $M_{2}$.

We may conjugate this in $G(\mathbb{C})$ to matrices of the form

$$
\gamma=\operatorname{diag}(s, a+i b, a-i b, t)
$$


in $A(\mathbb{C})$. Composing the roots of $A$ with this composition, we determine the positive imaginary root $\alpha_{M_{2}}=e_{2}-e_{3}$. We have $\Omega^{L M}=\left\{1, w_{2}\right\}$.

This gives

$$
\Phi_{M_{2}}\left(1, \Theta^{E}\right)=(-2)\left(\operatorname{dim}_{\mathbb{C}} V_{\lambda_{B}}^{M_{2}}-\operatorname{dim}_{\mathbb{C}} V_{\lambda_{B}^{\prime \prime}}^{M_{2}}\right),
$$

where $\lambda_{B}^{\prime \prime}=\frac{1}{2}(t-b-1, a+b-2,0, t-a+3) \in X^{*}(T)$. Note that

$$
\operatorname{pr}\left(\lambda_{B}^{\prime \prime}\right)=\frac{1}{4}(t+a-b-4, t+a+b-2, t-a-b+2, t-a+b+4) .
$$

The Weyl dimension formula yields

$$
\operatorname{dim}_{\mathbb{C}} V_{\lambda_{B}}^{M_{2}}=\frac{1}{2}(a-b) \quad \text { and } \quad \operatorname{dim}_{\mathbb{C}} V_{\lambda_{B}^{\prime \prime}}^{M_{2}}=\frac{1}{2}(a+b),
$$

and so

$$
S \Phi_{M_{2}}\left(1, e_{\pi_{G}}\right)=b \cdot \bar{v}\left(M_{2}\right)^{-1} .
$$

14.4. The term $S \Phi_{A}$. By (4-1), we have $\Phi_{A}\left(1, \Theta^{E}\right)=(-1)^{q(G)}\left|\Omega_{G}\right|=-8$, and so

$$
S \Phi_{A}\left(1, e_{\pi_{G}}\right)=4 \bar{v}(A)^{-1}
$$

\section{Computing $S \Phi_{M_{H}}$ for Levi subgroups of $H$}

Since $e_{\pi_{G}}^{H}=e_{\pi_{H}}+e_{\pi_{H}^{\prime}}$, we have

$$
\begin{aligned}
& S \Phi_{M_{H}}\left(1, e_{\pi_{G}}^{H}\right) \\
& \quad=(-1)^{q(G)}(-1)^{\operatorname{dim}\left(A_{M_{H}} / A_{H}\right)} \bar{v}\left(M_{H}\right)^{-1}\left(\Phi_{M_{H}}\left(1, \Theta_{\pi_{H}}\right)+\Phi_{M_{H}}\left(1, \Theta_{\pi_{H}^{\prime}}\right)\right) .
\end{aligned}
$$

15.1. The term $S \Phi_{H}\left(1, e_{\pi_{G}}^{H}\right)$. In this case $H$ has the elliptic torus $T_{H}$.

From (4-1), we obtain $\Phi_{H}\left(1, \Theta_{\pi_{H}}\right)=\operatorname{dim}_{\mathbb{C}} E_{H}$. To apply the dimension formula, we compute for instance $\left\langle\alpha_{1}, \lambda_{H}\right\rangle=a-1,\left\langle\alpha_{2}, \lambda_{H}\right\rangle=b-1$, and $\left\langle\alpha_{i}, \rho_{H}\right\rangle=1$.

We find that

$$
\Phi_{H}\left(1, \Theta^{E_{H}}\right)=\Phi_{H}\left(1, \Theta^{E_{H}^{\prime}}\right)=a b .
$$

Therefore

$$
S \Phi_{H}\left(1, e_{\pi_{G}}^{H}\right)=-2 \bar{v}(H)^{-1} a b .
$$

15.2. The term $S \Phi_{A^{H}}\left(1, e_{\pi_{G}}^{H}\right)$. From (4-1), we obtain

$$
\Phi_{A^{H}}\left(1, \Theta^{E_{H}}\right)=\Phi_{A^{H}}\left(1, \Theta^{E_{H}^{\prime}}\right)=4 .
$$

Therefore

$$
S \Phi_{A^{H}}\left(1, e_{\pi_{G}}^{H}\right)=-8 \bar{v}\left(A^{H}\right)^{-1} .
$$


15.3. The terms $S \Phi_{M_{H}}\left(1, e_{\pi_{G}}^{H}\right)$ for the intermediate Levi subgroups. For both $M=M_{H}^{1}$ and $M=M_{H}^{2}$, we have $\Omega_{G}=\Omega_{L} \Omega_{M}$, and so formula (4-1) becomes simply $\Phi_{M_{H}}\left(1, \Theta^{E_{H}}\right)=(-2) \operatorname{dim}_{\mathbb{C}} V_{\lambda_{H}}^{M_{H}}$ for both of these Levi subgroups.

We obtain

$$
\Phi_{M_{H}^{1}}\left(1, \Theta^{E_{H}}\right)=\Phi_{M_{H}^{2}}\left(1, \Theta^{E_{H}^{\prime}}\right)=-2 a
$$

and

$$
\Phi_{M_{H}^{2}}\left(1, \Theta^{E_{H}}\right)=\Phi_{M_{H}^{1}}\left(1, \Theta^{E_{H}^{\prime}}\right)=-2 b .
$$

Therefore

$$
S \Phi_{M_{H}^{1}}\left(1, e_{\pi_{G}}^{H}\right)=S \Phi_{M_{H}^{2}}\left(1, e_{\pi_{G}}^{H}\right)=-2 \bar{v}\left(M_{H}^{1}\right)^{-1}(a+b) .
$$

\section{Final form: $\gamma$ central}

Recall that $G=\mathrm{GSp}_{4}$. For the convenience of the reader, we recall the setup.

Let $a$ and $b$ be odd integers with $a>b>0$, and $t$ an even integer. Consider the Langlands parameter $\varphi_{G}: W_{\mathbb{R}} \rightarrow{ }^{L} G$ given by

$$
\varphi_{G}(z)=|z|^{t} \operatorname{diag}\left(\theta(z)^{a}, \theta(z)^{b}, \theta(z)^{-b}, \theta(z)^{-a}\right) \times z \quad \text { and } \quad \varphi_{G}(\tau)=J \times \tau .
$$

Let $\pi_{G}$ be the discrete series representation $\pi\left(\varphi_{G}, B_{T}\right)$ of $G(\mathbb{R})$ as in Section 2.2. Write $\pi_{G}^{\prime}$ for the other representation in $\Pi\left(\varphi_{G}\right)$.

Put $f_{\infty} d g_{\infty}=e_{\pi_{G}}$ as in Section 2.4 for $\pi_{G}$ and any measure $f^{\infty} d g_{f}$ on $G\left(\mathbb{A}_{f}\right)$. Let $f d g=e_{\pi_{G}} f^{\infty} d g_{f}$, a measure on $G(\mathbb{A})$. By the theory of endoscopic transfer there is a matching measure $f^{H} d h$ on $H(\mathbb{A})$, where $H$ is the elliptic endoscopic group $P\left(\mathrm{GL}_{2} \times \mathrm{GL}_{2}\right)$ discussed above.

If $z \in A_{G}(\mathbb{Q})$, then $\sum_{M} S T_{g}(f d g, z, M)$ is given by the product of $\lambda_{E}(z)=z^{t}$ with

$$
\begin{aligned}
-\frac{1}{24} a b(a+b)(a-b) \bar{v}(G)^{-1} f^{\infty}(z)+ & \frac{1}{2}(a-b) \bar{v}\left(M_{1}\right)^{-1} f_{M_{1}}^{\infty}(z) \\
& +\frac{1}{2} b \bar{v}\left(M_{2}\right)^{-1} f_{M_{2}}^{\infty}(z)+\frac{1}{2} \bar{v}(A)^{-1} f_{A}^{\infty}(z) .
\end{aligned}
$$

If $z=\left(z_{1}, z_{2}\right) \in A_{H}(\mathbb{Q})$, then $\sum_{M_{H}} S T_{g}\left(f^{H} d h, z, M_{H}\right)$ is given by the product of $\lambda_{E_{H}}(z)=\left(z_{1} z_{2}\right)^{t}$ with

$$
-4 a b \bar{v}(H)^{-1} f^{H, \infty}(z)-2(a+b) \bar{v}\left(M_{H}^{1}\right)^{-1} f_{M_{2}}^{\infty}(z)-2 \bar{v}\left(A^{H}\right)^{-1} f_{A^{H}}^{\infty}(z) .
$$

\section{The case $\Gamma=\mathrm{Sp}_{4}(\mathbb{Z})$}

Let $f^{\infty} d g_{f}=e_{K_{0}}$, where $K_{0}=G\left(\mathbb{O}_{f}\right)$. Here $d g_{f}$ is an arbitrary Haar measure on $G\left(\mathbb{A}_{f}\right)$, so that $d g=d g_{f} d g_{\infty}$ is the Tamagawa measure on $G(\mathbb{A})$. 
17.1. Central terms in $\boldsymbol{G}$. Note that $f_{M}^{\infty}(z)=0$ for all $z \in Z(\mathbb{Q})$ unless $z= \pm 1$, and that $f_{M}^{\infty}(1)=f_{M}^{\infty}(-1)$ for all Levi subgroups $M$.

First we compute $S T_{g}(f d g, \pm 1, G)$. We have

$$
\begin{aligned}
-\frac{1}{2^{3} 3} a b(a+b)(a-b) & \bar{v}(G)^{-1} f^{\infty}( \pm 1) \\
= & -\frac{1}{2^{3} 3} a b(a+b)(a-b) \tau(G)^{-1} d(G)^{-1} \chi_{K_{0}}(G) \\
= & 2^{-10} 3^{-3} 5^{-1} a b(a+b)(a-b) .
\end{aligned}
$$

Next we treat the $\pm 1 \in M_{i}$ terms, for the intermediate Levi subgroups. We have

$$
\begin{aligned}
& S T_{g}\left(f d g, \pm 1, M_{1}\right)=\frac{1}{2}(a-b) \bar{v}\left(M_{1}\right)^{-1} f_{M_{1}}^{\infty}( \pm 1)=-2^{-5} 3^{-1}(a-b), \\
& S T_{g}\left(f d g, \pm 1, M_{2}\right)=\frac{1}{2} b \bar{v}\left(M_{2}\right)^{-1} f_{M_{2}}^{\infty}( \pm 1)=-2^{-5} 3^{-1} b .
\end{aligned}
$$

Next we treat the $\pm 1 \in A$ terms. We have $f_{A}(1)=\operatorname{vol}_{d a_{f}}\left(K \cap A\left(\mathbb{A}_{f}\right)\right)^{-1}$, which is 1 . Moreover we take Lebesgue measure on $A(\mathbb{R})$ so that $\bar{v}(A)=8$. It follows that

$$
S T_{g}(f d g, \pm 1, A)=\frac{1}{2} \bar{v}(A)^{-1} f_{A}^{\infty}( \pm 1)=2^{-4} .
$$

Doubling these terms to account for both central elements, we compute

$$
\begin{aligned}
& \sum_{z, M} S T_{g}(f d g, z, M) \\
& \quad=2^{-9} 3^{-3} 5^{-1} a b(a+b)(a-b)-2^{-4} 3^{-1}(a-b)-2^{-4} 3^{-1} b+2^{-3} .
\end{aligned}
$$

17.2. Central terms in $\boldsymbol{H}$. By the fundamental lemma ([Hales 1997; Weissauer 2009] for $\mathrm{GSp}_{4}$, and of course [Ngô 2010] in general), we may write $\left(e_{K_{0}}\right)^{H}=e_{K_{H}}$, where $K_{H}=H\left(\mathscr{O}_{f}\right)$. Thus $\left(f^{\infty}\right)_{M}^{H}(z)=0$ for all $z \in H(\mathbb{Q})$ unless $z=(1, \pm 1)$, and

$$
f_{M}^{H \infty}(1,1)=f_{M}^{H \infty}(1,-1)
$$

for all Levi subgroups $M=M_{H}$ of $H$.

The only nontrivial factors in the formula of Theorem 2 are $|\operatorname{ker} \rho(\mathbb{Q})|=2$, $\left[H(\mathbb{R}): H(\mathbb{R})_{+}\right]=4$, and $\chi_{\mathrm{alg}}\left(H^{\mathrm{sc}}(\mathbb{Z})\right)$. Note that $H^{\mathrm{sc}}=\mathrm{SL}_{2} \times \mathrm{SL}_{2}$.

Therefore

$$
\chi_{K_{H}}(H)=2^{-1} \chi_{\mathrm{alg}}\left(\mathrm{SL}_{2}(\mathbb{Z})\right)^{2}=2^{-5} 3^{-2} .
$$

We conclude that

$$
S T_{g}\left(f^{H} d h,(1, \pm 1), H\right)=-4 a b \bar{v}(H)^{-1} \operatorname{vol}\left(K_{H}\right)^{-1}=-2^{-4} 3^{-2} a b .
$$


Next we find that

$$
\begin{aligned}
\sum_{i=1}^{2} S T_{g}\left(f^{H} d h,(1, \pm 1), M_{i}^{H}\right) & =-2(a+b) \bar{v}\left(M_{1}^{H}\right)^{-1} \operatorname{vol}\left(K_{M}\right)^{-1} \\
& =2^{-3} 3^{-1}(a+b) .
\end{aligned}
$$

Finally, we have

$$
S T_{g}\left(f^{H} d h,(1, \pm 1), A^{H}\right)=-2 \bar{v}(A)^{-1} \operatorname{vol}\left(K_{A}\right)^{-1}=-2^{-2} .
$$

Multiplying by $\iota(G, H)=4^{-1}$ and then doubling to account for both central elements, we compute

$$
\iota(G, H) \sum_{z, M_{H}} S T_{g}\left(f^{H} d h, z, M_{H}\right)=-2^{-5} 3^{-2} a b+2^{-4} 3^{-1}(a+b)-2^{-3} .
$$

\section{Comparison}

As mentioned in the introduction, Wakatsuki [ $\geq 2012 ; 2012]$ has used the Selberg trace formula and Arthur's $L^{2}$-Lefschetz number formula to compute the discrete series multiplicities $m_{\text {disc }}(\pi, \Gamma)$ for $\pi$ both holomorphic and large discrete series representations for $\mathrm{Sp}_{4}(\mathbb{R})$, and for many cases of arithmetic subgroups $\Gamma$. We will compare our formula to his when $\Gamma$ is the full modular group. (Note that if $\pi$ is a discrete series representation of $\mathrm{GSp}_{4}(\mathbb{R})$ with trivial central character, and $\pi_{1}$ is its restriction to $\operatorname{Sp}_{4}(\mathbb{R})$, then $m_{\text {disc }}(\pi, \Gamma)=m_{\text {disc }}\left(\pi_{1}, \Gamma_{1}\right)$, where $\Gamma_{1}=\operatorname{Sp}_{4}(\mathbb{Z})$.) Since he is using the Selberg trace formula, his formula breaks into contributions from each conjugacy class in $\Gamma$. In particular, he identifies the central-unipotent contributions $H_{1}^{\mathrm{Hol}}$ and $H_{1}^{\text {Large }}$ to $m_{\text {disc }}\left(\pi_{G}\right)$ and $m_{\text {disc }}\left(\pi_{G}^{\prime}\right)$, respectively. Namely,

$$
\begin{aligned}
H_{1}^{\mathrm{Hol}} & =2^{-9} 3^{-3} 5^{-1} a b(a-b)(a+b)-2^{-5} 3^{-2} a b+2^{-4} 3^{-1} b, \\
H_{1}^{\mathrm{Large}} & =2^{-9} 3^{-3} 5^{-1} a b(a-b)(a+b)+2^{-5} 3^{-2} a b-2^{-3} 3^{-1} b+2^{-2} .
\end{aligned}
$$

(To translate from his notation to ours, use $j=b-1$ and $k=\frac{1}{2}(a-b)+2$.)

Comparing these formulas to our formulas above, we observe

$$
H_{1}^{\mathrm{Hol}}=\sum_{M} S T_{g}(f d g, \pm 1, M)+\iota(G, H) \sum_{M_{H}} S T_{g}\left(f^{H} d h,(1, \pm 1), M_{H}\right)
$$

when $f d g=e_{\pi_{G}} e_{K_{0}}$ and

$$
H_{1}^{\mathrm{Large}}=\sum_{M} S T_{g}(f d g, \pm 1, M)+\iota(G, H) \sum_{M_{H}} S T_{g}\left(f^{H} d h,(1, \pm 1), M_{H}\right) .
$$

when $f d g=e_{\pi_{G}^{\prime}} e_{K_{0}}$.

This proves Theorem 1 . 


\section{Acknowledgments}

This paper is founded on my thesis under the direction of Robert Kottwitz. I would like to thank him for his continual help with this project. I am also indebted to Satoshi Wakatsuki for predicting Theorem 1, and for much useful correspondence. I would also like to thank Ralf Schmidt for helpful conversations.

\section{References}

[Arthur 1988a] J. Arthur, "The invariant trace formula, I: Local theory", J. Amer. Math. Soc. 1:2 (1988), 323-383. MR 89e:22029 Zbl 0682.10021

[Arthur 1988b] J. Arthur, "The invariant trace formula, II: Global theory", J. Amer. Math. Soc. 1:3 (1988), 501-554. MR 89j:22039 Zbl 0667.10019

[Arthur 1989] J. Arthur, “The $L^{2}$-Lefschetz numbers of Hecke operators", Invent. Math. 97:2 (1989), 257-290. MR 91i:22024 Zbl 0692.22004

[Arthur 2001] J. Arthur, "A stable trace formula, II: Global descent", Invent. Math. 143:1 (2001), 157-220. MR 2002m:11043 Zbl 0978.11025

[Arthur 2002] J. Arthur, "A stable trace formula, I: General expansions", J. Inst. Math. Jussieu 1:2 (2002), 175-277. MR 2004b:11066 Zbl 1040.11038

[Arthur 2003] J. Arthur, "A stable trace formula, III: Proof of the main theorems", Ann. of Math. (2) 158:3 (2003), 769-873. MR 2004m:11079 Zbl 1051.11027

[Arthur 2005] J. Arthur, "An introduction to the trace formula", pp. 1-263 in Harmonic analysis, the trace formula, and Shimura varieties (Toronto, 2003), edited by J. Arthur et al., Clay Math. Proc. 4, Amer. Math. Soc., Providence, RI, 2005. MR 2007d:11058 Zbl 1152.11021

[Borel 1979] A. Borel, “Automorphic L-functions", pp. 27-61 in Automorphic forms, representations and L-functions (Corvallis, OR, 1977), vol. 2, edited by A. Borel and W. Casselman, Proc. Sympos. Pure Math. 33, Amer. Math. Soc., Providence, RI, 1979. MR 81m:10056 Zbl 0412.10017

[Clozel and Delorme 1984] L. Clozel and P. Delorme, "Le théorème de Paley-Wiener invariant pour les groupes de Lie réductifs", Invent. Math. 77:3 (1984), 427-453. MR 86b:22015 Zbl 0584.22005

[Clozel and Delorme 1990] L. Clozel and P. Delorme, "Le théorème de Paley-Wiener invariant pour les groupes de Lie réductifs, II”, Ann. Sci. École Norm. Sup. (4) 23:2 (1990), 193-228. MR 91g:22013 Zbl 0724.22012

[Deligne 1979] P. Deligne, "Variétés de Shimura: interprétation modulaire, et techniques de construction de modèles canoniques", pp. 247-289 in Automorphic forms, representations and Lfunctions (Corvallis, OR, 1977), vol. 2, edited by A. Borel and W. Casselman, Proc. Sympos. Pure Math. 33, Amer. Math. Soc., Providence, RI, 1979. MR 81i:10032 Zbl 0437.14012

[Goresky et al. 1997] M. Goresky, R. E. Kottwitz, and R. MacPherson, "Discrete series characters and the Lefschetz formula for Hecke operators", Duke Math. J. 89:3 (1997), 477-554. MR 99e: 11064a Zbl 0888.22011

[Hales 1997] T. C. Hales, "The fundamental lemma for Sp(4)”, Proc. Amer. Math. Soc. 125:1 (1997), 301-308. MR 97c:22020 Zbl 0876.22022

[Harder 1971] G. Harder, "A Gauss-Bonnet formula for discrete arithmetically defined groups", Ann. Sci. École Norm. Sup. (4) 4:3 (1971), 409-455. MR 46 \#8255 Zbl 0232.20088

[Kottwitz 1983] R. E. Kottwitz, "Sign changes in harmonic analysis on reductive groups", Trans. Amer. Math. Soc. 278:1 (1983), 289-297. MR 84i:22012 Zbl 0538.22010 
[Kottwitz 1984] R. E. Kottwitz, "Stable trace formula: Cuspidal tempered terms", Duke Math. J. 51:3 (1984), 611-650. MR 85m:11080 Zbl 0576.22020

[Kottwitz 1986] R. E. Kottwitz, "Stable trace formula: Elliptic singular terms", Math. Ann. 275:3 (1986), 365-399. MR 88d:22027 Zbl 0577.10028

[Kottwitz 1988] R. E. Kottwitz, “Tamagawa numbers”, Ann. of Math. (2) 127:3 (1988), 629-646. MR 90e:11075 Zbl 0678.22012

[Kottwitz 1990] R. E. Kottwitz, "Shimura varieties and $\lambda$-adic representations", pp. 161-209 in Automorphic forms, Shimura varieties, and L-functions (Ann Arbor, MI, 1988), vol. 1, edited by L. Clozel and J. S. Milne, Perspect. Math. 10, Academic Press, Boston, 1990. MR 92b:11038 Zbl 0743.14019

[Kottwitz 2005] R. E. Kottwitz, "Harmonic analysis on reductive p-adic groups and Lie algebras", pp. 393-522 in Harmonic analysis, the trace formula, and Shimura varieties (Toronto, 2003), edited by J. Arthur et al., Clay Math. Proc. 4, Amer. Math. Soc., Providence, RI, 2005. MR 2006m:22016 Zbl 1106.22013

[Kottwitz $\geq 2012$ ] R. E. Kottwitz, "Stable version of Arthur's formula", preprint.

[Kottwitz and Shelstad 1999] R. E. Kottwitz and D. Shelstad, Foundations of twisted endoscopy, Astérisque 255, Sociéte Mathématique de France, Paris, 1999. MR 2000k:22024 Zbl 0958.22013

[Langlands 1976] R. P. Langlands, On the functional equations satisfied by Eisenstein series, Lecture Notes in Mathematics 544, Springer, Berlin, 1976. MR 58 \#28319 Zbl 0332.10018

[Langlands 1979] R. P. Langlands, "Stable conjugacy: definitions and lemmas", Canad. J. Math. 31:4 (1979), 700-725. MR 82j:10054 Zbl 0421.12013

[Langlands 1983] R. P. Langlands, Les débuts d'une formule des traces stable, Publications Mathématiques 13, Université de Paris VII, 1983. MR 85d:11058 Zbl 0532.22017

[Langlands and Shelstad 1987] R. P. Langlands and D. Shelstad, "On the definition of transfer factors", Math. Ann. 278:1-4 (1987), 219-271. MR 89c:11172 Zbl 0644.22005

[Milne 2005] J. S. Milne, "Introduction to Shimura varieties", pp. 265-378 in Harmonic analysis, the trace formula, and Shimura varieties (Toronto, 2003), edited by J. Arthur et al., Clay Math. Proc. 4, Amer. Math. Soc., Providence, RI, 2005. MR 2006m:11087 Zbl 1148.14011

[Morel 2010] S. Morel, On the cohomology of certain noncompact Shimura varieties, Annals of Mathematics Studies 173, Princeton University Press, 2010. MR 2011b:11073 Zbl 1233.11069

[Ngô 2010] B. C. Ngô, "Le lemme fondamental pour les algèbres de Lie", Publ. Math. Inst. Hautes Études Sci. 111:1 (2010), 1-169. MR 2011h:22011 Zbl 1200.22011

[Ono 1966] T. Ono, “On Tamagawa numbers”, pp. 122-132 in Algebraic groups and discontinuous subgroups (Boulder, CO, 1965), edited by A. Borel and G. D. Mostow, Proc. Sympos. Pure Math. 9, Amer. Math. Soc., Providence, RI, 1966. MR 35 \#191 Zbl 0223.20050

[Pitale and Schmidt 2009] A. Pitale and R. Schmidt, "Bessel models for lowest weight representations of GSp(4, R)", Int. Math. Res. Not. 2009:7 (2009), 1159-1212. MR 2010h:11084 Zbl 05553685

[Roberts and Schmidt 2007] B. Roberts and R. Schmidt, Local newforms for GSp(4), Lecture Notes in Mathematics 1918, Springer, Berlin, 2007. MR 2008g:11080 Zbl 1126.11027

[Satake 1980] I. Satake, Algebraic structures of symmetric domains, Kanô Memorial Lectures 4, Iwanami Shoten, Tokyo, 1980. MR 82i:32003 Zbl 0483.32017

[Selberg 1956] A. Selberg, "Harmonic analysis and discontinuous groups in weakly symmetric Riemannian spaces with applications to Dirichlet series", J. Indian Math. Soc. (N.S.) 20 (1956), 47-87. MR 19,531g Zbl 0072.08201 
[Serre 1971] J.-P. Serre, "Cohomologie des groupes discrets", pp. 77-169 in Prospects in mathematics (Princeton, 1970), edited by F. Hirzebruch et al., Ann. of Math. Studies 70, Princeton University Press, 1971. MR 52 \#5876 Zbl 0235.22020

[Shelstad 1982] D. Shelstad, “L-indistinguishability for real groups”, Math. Ann. 259:3 (1982), 385430. MR 84c:22017 Zbl 0506.22014

[Spallone 2004] S. T. Spallone, Arthur's trace formula for $\mathrm{SO}(5)$ and individual discrete series matrix coefficients, thesis, Univ. Chicago, 2004, available at http://www.math.ou.edu/ sspallone/ Papers/Dissertation.pdf.

[Spallone 2009] S. T. Spallone, "Stable discrete series characters at singular elements", Canad. J. Math. 61:6 (2009), 1375-1382. MR 2011c:22025 Zbl 1180.22019

[Spallone 2011] S. T. Spallone, "On the trace formula approach to discrete series multiplicities", pp. 1-13 in Automorphic forms, trace formulas and zeta functions (Kyoto, 2011), edited by Y. Gon and T. Moriyama, RIMS Kokyuroku 1767, Res. Inst. Math. Sci., Kyoto, 2011.

[Springer 1979] T. A. Springer, "Reductive groups", pp. 3-27 in Automorphic forms, representations and L-functions (Corvallis, OR, 1977), vol. 1, edited by A. Borel and W. Casselman, Proc. Sympos. Pure Math. 33, Amer. Math. Soc., Providence, RI, 1979. MR 80h:20062 Zbl 0416.20034

[Tsushima 1983] R. Tsushima, "An explicit dimension formula for the spaces of generalized automorphic forms with respect to Sp(2, ZZ)”, Proc. Japan Acad. Ser. A Math. Sci. 59:4 (1983), 139-142. MR 85a:11011 Zbl 0513.10025

[Tsushima 1997] R. Tsushima, "Dimension formula for the spaces of Siegel cusp forms and a certain exponential sum”, Mem. Inst. Sci. Tech. Meiji Univ. 36 (1997), 1-56.

[Wakatsuki 2012] S. Wakatsuki, "Dimension formulas for spaces of vector-valued Siegel cusp forms of degree two", J. Number Theory 132:1 (2012), 200-253. MR 2843308 Zbl 05966274

[Wakatsuki $\geq 2012$ ] S. Wakatsuki, "Multiplicity formulas for discrete series representations in $L^{2}(\Gamma \backslash \operatorname{Sp}(2, \mathbb{R}))$ ", preprint.

[Wallach 1984] N. R. Wallach, "On the constant term of a square integrable automorphic form", pp. 227-237 in Operator algebras and group representations (Neptun, 1980), vol. 2, edited by G. Arsene, Monogr. Stud. Math. 18, Pitman, Boston, 1984. MR 86i:22029 Zbl 0554.22004

[Warner 1972] G. Warner, Harmonic analysis on semi-simple Lie groups, I, Grundlehren Math. Wiss. 188, Springer, New York, 1972. MR 58 \#16979 Zbl 0265.22020

[Weissauer 2009] R. Weissauer, "A special case of the fundamental lemma, I-IV", pp. 211-320 in Endoscopy for GSp(4) and the cohomology of Siegel modular threefolds, Lecture Notes in Mathematics 1968, Springer, Berlin, 2009.

Received April 26, 2011. Revised December 5, 2011.

\section{STEVEN SPALLONE}

SCHOOL OF MATHEMATICS

TATA INSTITUTE OF FUNDAMENTAL RESEARCH

HOMI BHABHA RD

COLABA

Mumbai 400005

INDIA

sspallone@gmail.com 


\title{
PACIFIC JOURNAL OF MATHEMATICS
}

\author{
http://pacificmath.org \\ Founded in 1951 by \\ E. F. Beckenbach (1906-1982) and F. Wolf (1904-1989)
}

\section{EDITORS}

V. S. Varadarajan (Managing Editor)

Department of Mathematics

University of California

Los Angeles, CA 90095-1555

pacific@math.ucla.edu

Vyjayanthi Chari

Department of Mathematics

University of California

Riverside, CA 92521-0135

chari@math.ucr.edu

\section{Robert Finn}

Department of Mathematics Stanford University

Stanford, CA 94305-2125

finn@math.stanford.edu

Kefeng Liu

Department of Mathematics

University of California

Los Angeles, CA 90095-1555

liu@math.ucla.edu
Darren Long

Department of Mathematics

University of California

Santa Barbara, CA 93106-3080

long@math.ucsb.edu

Jiang-Hua Lu

Department of Mathematics

The University of Hong Kong

Pokfulam Rd., Hong Kong jhlu@maths.hku.hk

Alexander Merkurjev

Department of Mathematics

University of California

Los Angeles, CA 90095-1555

merkurev@math.ucla.edu
Sorin Popa

Department of Mathematics University of California

Los Angeles, CA 90095-1555 popa@math.ucla.edu

Jie Qing

Department of Mathematics

University of California

Santa Cruz, CA 95064

qing@cats.ucsc.edu

Jonathan Rogawski

Department of Mathematics

University of California

Los Angeles, CA 90095-1555

jonr@math.ucla.edu

\section{PRODUCTION}

pacific@math.berkeley.edu

\section{SUPPORTING INSTITUTIONS}

ACADEMIA SINICA, TAIPEI

CALIFORNIA INST. OF TECHNOLOGY INST. DE MATEMÁTICA PURA E APLICADA KEIO UNIVERSITY

MATH. SCIENCES RESEARCH INSTITUTE NEW MEXICO STATE UNIV.

OREGON STATE UNIV.

\author{
STANFORD UNIVERSITY \\ UNIV. OF BRITISH COLUMBIA \\ UNIV. OF CALIFORNIA, BERKELEY \\ UNIV. OF CALIFORNIA, DAVIS \\ UNIV. OF CALIFORNIA, LOS ANGELES \\ UNIV. OF CALIFORNIA, RIVERSIDE \\ UNIV. OF CALIFORNIA, SAN DIEGO \\ UNIV. OF CALIF., SANTA BARBARA
}

\author{
UNIV. OF CALIF., SANTA CRUZ \\ UNIV. OF MONTANA \\ UNIV. OF OREGON \\ UNIV. OF SOUTHERN CALIFORNIA \\ UNIV. OF UTAH \\ UNIV. OF WASHINGTON \\ WASHINGTON STATE UNIVERSITY
}

These supporting institutions contribute to the cost of publication of this Journal, but they are not owners or publishers and have no responsibility for its contents or policies.

See inside back cover or pacificmath.org for submission instructions.

The subscription price for 2012 is US \$420/year for the electronic version, and \$485/year for print and electronic.

Subscriptions, requests for back issues from the last three years and changes of subscribers address should be sent to Pacific Journal of Mathematics, P.O. Box 4163, Berkeley, CA 94704-0163, U.S.A. Prior back issues are obtainable from Periodicals Service Company, 11 Main Street, Germantown, NY 12526-5635. The Pacific Journal of Mathematics is indexed by Mathematical Reviews, Zentralblatt MATH, PASCAL CNRS Index, Referativnyi Zhurnal, Current Mathematical Publications and the Science Citation Index.

The Pacific Journal of Mathematics (ISSN 0030-8730) at the University of California, c/o Department of Mathematics, 969 Evans Hall, Berkeley, CA 94720-3840, is published monthly except July and August. Periodical rate postage paid at Berkeley, CA 94704, and additional mailing offices. POSTMASTER: send address changes to Pacific Journal of Mathematics, P.O. Box 4163, Berkeley, CA 94704-0163.

PJM peer review and production are managed by EditFLOW ${ }^{\mathrm{TM}}$ from Mathematical Sciences Publishers.

PUBLISHED BY PACIFIC JOURNAL OF MATHEMATICS

at the University of California, Berkeley 94720-3840

A NON-PROFIT CORPORATION

Typeset in LATEX

Copyright $(02012$ by Pacific Journal of Mathematics 


\title{
PACIFIC JOURNAL OF MATHEMATICS
}

\author{
Volume $256 \quad$ No. $2 \quad$ April 2012
}

O-operators on associative algebras and associative Yang-Baxter equations

Chengming BAI, Li GuO and Xiang Ni

Botany of irreducible automorphisms of free groups

291

THIERRY COULBOIS and ARNAUd HILION

A note on inverse curvature flows in asymptotically Robertson-Walker 309 spacetimes

Claus GerhardT

Total curvature of graphs after Milnor and Euler

Robert GULLIVER and SUMIO YAMADA

Entire solutions of Donaldson's equation

WEIYONG HE

Energy identity and removable singularities of maps from a Riemann surface with tension field unbounded in $L^{2}$

YONG LUO

Quotients by actions of the derived group of a maximal unipotent subgroup

\section{DMITRI I. PANYUSHEV}

Invariants of totally real Lefschetz fibrations

NERMIN SALEPCI

Stable trace formulas and discrete series multiplicities

STEVEN SPALLONE

Small covers and the Halperin-Carlsson conjecture

\section{YU}

